UC-706

Issued: November 1995

Stochastic Interpenetration of Fluids

Michael J. Steinkamp

Timothy T. Clark

Francis H. Harlow 


\section{DISCLAIMER}

This report was prepared as an account of work sponsored by an agency of the United States Government. Neither the United States Government nor any agency thereof, nor any of their employees, make any warranty, express or implied, or assumes any legal liability or responsibility for the accuracy, completeness, or usefulness of any information, apparatus, product, or process disclosed, or represents that its use would not infringe privately owned rights. Reference herein to any specific commercial product, process, or service by trade name, trademark, manufacturer, or otherwise does not necessarily constitute or imply its endorsement, recommendation, or favoring by the United States Government or any agency thereof. The views and opinions of authors expressed herein do not necessarily state or reflect those of the United States Government or any agency thereof. 


\section{DISCLAIMER}

Portions of this document may be illegible in electronic image products. Images are produced from the best available original document. 


\title{
STOCHASTIC INTERPENETRATION OF FLUIDS
}

\author{
by
}

Michael J. Steinkamp, Timothy T. Clark, and Francis H. Harlow

\begin{abstract}
We describe a spectral approach to the investigation of fluid instability, generalized turbulence, and the interpenetration of fluids across an interface. The technique also applies to a single fluid with large variations in density. Departures of fluctuating velocity components from the local mean are far subsonic, but the mean Mach number can be large. Validity of the description is demonstrated by comparisons with experiments on turbulent mixing due to the late stages of Rayleigh-Taylor instability, when the dynamics become approximately self-similar in response to a constant body force. Generic forms for anisotropic spectral structure are described and used as a basis for deriving spectrally integrated moment equations that can be incorporated into computer codes for scientific and engineering analyses.
\end{abstract}

\section{INTRODUCTION}

Turbulence occurs in many circumstances of fluid flow, being driven or sustained by the conversion of large-scale mean flow energy to intermediate-scale fluctuations and dissipated by the entropy-increasing process of cascade to small scales, ultimately to the molecular level where it is manifested in the form of heat.

Our goal is to investigate the nature of turbulence by means of extended transport approaches, using two-point (spectral) techniques. In particular we seek "generic" spectral form functions by which to describe the turbulence structure, especially in circumstances of inhomogeneous, anisotropic drive. These functions may be exact or approximate, depending on circumstances to be described below. They are usually revealed in greatest clarity under circumstances that are described by self-similar combinations of the physical and spectral variables, but they may also 
occur to a significant level of approximation in localized regions that are continually approaching self-similarity despite the shifting nature of the mean-flow drive.

This work is focused on flows with large variations in fluid density (e.g., two-field fluid interpenetration). The starting point for analysis is the set of Navier-Stokes equations, for which we assume relevance in our investigations, even in the presence of sharp density variations between fluids. Models for two-field analysis with drag representations for momentum exchange can also be used and are discussed by Steinkamp (1995).

In this report departures from mean flow are included in the stochastic concept of turbulence. Reynolds decomposition into mean and fluctuating parts is carried out in the spirit of this generalized concept, which is meaningful despite arbitrariness as to which scales are identified as mean flow and which are identified as fluctuations. (We actually derive much useful guidance by shifting our viewpoint in this regard as discussed in Section III.)

Insertion of Reynolds decomposition into the Navier-Stokes equations and ensemble averaging, using mass-weighted averages, leads to an unclosed hierarchy of transport equations for correlations at all orders. Written for a single point in space and supplemented by closure derivations or postulates, the results have proved useful for describing numerous circumstances of two-fluid turbulence (Andronov et al., 1982; Besnard, Harlow, and Rauenzahn, 1987). [This model proposed by Besnard et al. (1987) is hereafter referred to as the BHR model.] There are, however, some significant elements missing from this approach.

A clue to these missing elements lies in considering the much better known turbulence transport equations for constant-density fluid flow. It has long been recognized that a single-point transport equation for Reynolds stress must be supplemented by a transport equation for an auxiliary quantity, often chosen to be the dissipation tensor, $\varepsilon_{\mathrm{ij}}$, or its contraction, $\varepsilon$. Previously identified (Daly and Harlow, 1970) as the Reynolds-stress sink caused by molecular viscosity, $\varepsilon$ is more accurately associated with the cascade flux of energy from low to high wave numbers. Recognizing the necessity for a transport equation of this dissipation tensor provides motivation for considering a two-point generalization of the Reynolds stress, from which we can derive the spectral transport equations by Fourier transformation. This spectral representation does not require a supplemental transport equation for scale because it describes the continuously evolving distribution of Reynolds stress across all wave numbers. In particular it confirms the interpretation of $\varepsilon$ as the flux from the dominant lower-wave-number parts of the spectrum through a more-orless inertial (or Kolmogorov) middle range to high wave numbers. Viscous dissipation may occur at even higher wave numbers at a rate that is not necessarily in equilibrium with the cascade flux. A transport equation for $\varepsilon$ can be derived as a moment of the full spectral equations provided that an appropriate spectral form can be discovered for the spectral tensor, as discussed below. 
With large density differences in the fluid, the spectral analysis becomes much more complicated, especially because we include density variations that arise from the interpenetration of two completely immiscible materials: bubbles in a liquid, solid grains in a gas, or two immiscible fluids interacting in a general configuration of swirling filaments and droplets. The Navier-Stokes equations are assumed to be relevant for all three of these cases. For the case of solid grains dispersed in a gas, the regime of validity for this study is limited to the case where the grains of solids are small enough to treat the cloud of dispersed phase particles in the gas as a separate fluid.

At this stage of theoretical development, the attainment of a rigorous formulation even for constant density flows is not possible. During the last three decades of constant-density turbulence-transport developments, the derivations have had to combine empiricism with rigor, and the same is true for the variable-density extensions. Constraints (dimensionality, preservation of conservation, tensor form, Galilean invariance, simplicity of form, and similar general considerations) serve to limit the choices for modeling. Comparisons with experiments likewise furnish guidance.

In this report we describe a spectral turbulence transport formulation for variable-density turbulence in circumstances of strong inhomogeneity and anisotropy. We show that moments of these equations introduce some auxiliary single-point variables. These are some of the elements that are missing from previous single-point formulations for variable-density turbulence (e.g., the BHR model). We also describe some comparisons with experiments of the spectral formulation and indicate numerous directions for refinement, analogous to those that are required for constantdensity turbulence transport.

The family of model-development work into which these variable-density turbulence activities fit is shown in the diagram of Fig. I.1. The starting point for all directions is the Navier-Stokes equations.

Guidance for our spectral model (SCH model) is taken from these three branches of transport modeling, the simple (local) closures describing transfers in $\mathbf{k}$-space from the two-point branch [Besnard, Harlow, Rauenzahn, and Zemach, 1990; Clark and Spitz, 1995]; the single-point, single fluid, turbulence transport models; and the single-point, two-field models. Another branch from the two-point box could be shown that handles the transfers of energy in $\mathbf{k}$-space with much detail, but no use is made of that approach for the $\mathrm{SCH}$ model. Likewise other branches that exist are not shown since the ancestry of the $\mathrm{SCH}$ model is composed of only these three branches.

Another branch that evolves directly from the Navier-Stokes equations leads to the two-field (or multifield) flow equations. Explicit modeling describes the principal interaction between discrete entities and fluid in terms of drag. Within the two-field formulation, the fluid interpenetration is described by terms in the equations that contain no contributions from 


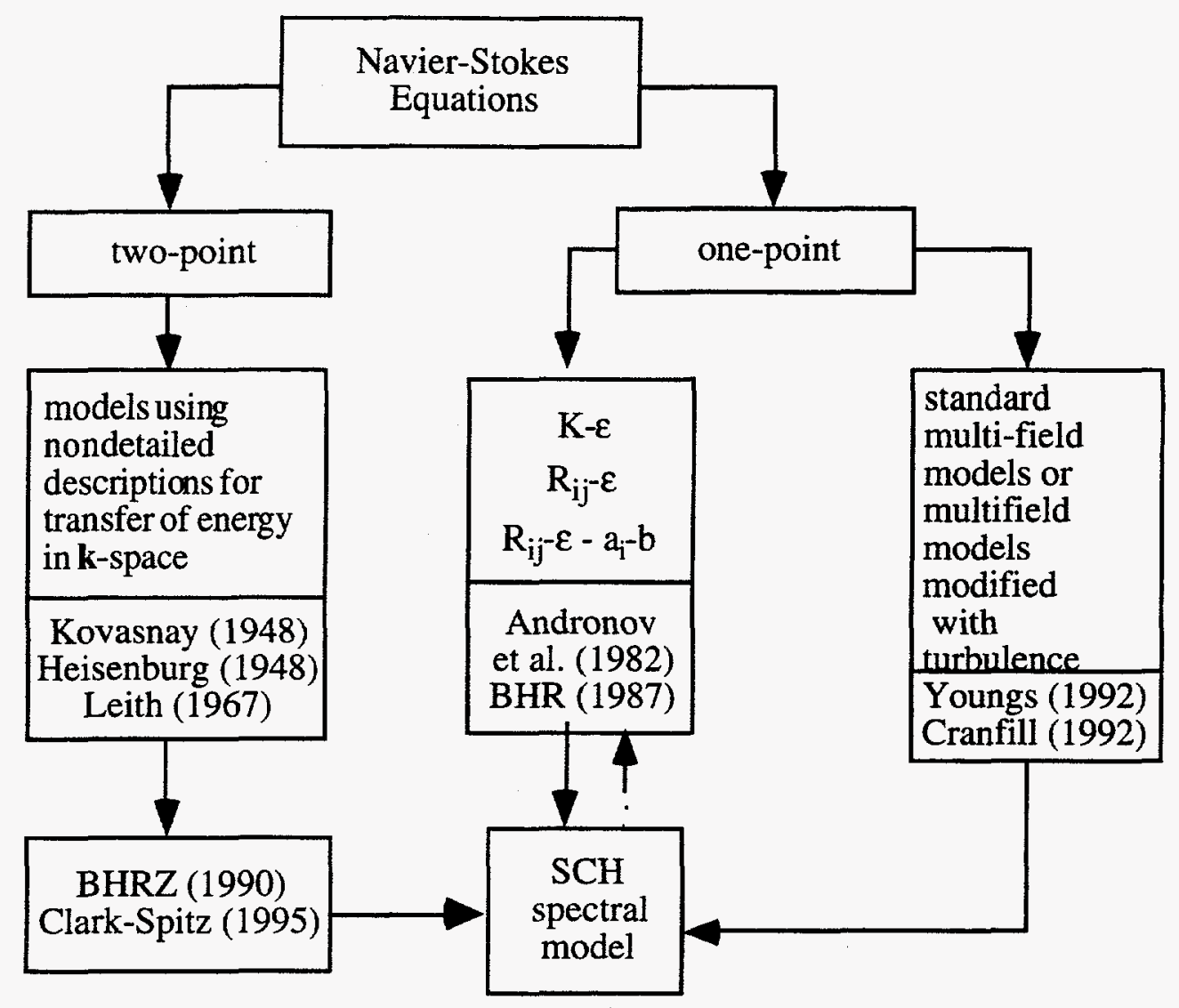

Fig. I.1. Flow chart defining the ancestry of the SCH spectral transport model .

fluctuations away from the mean within each field. This interpenetration is an "ordered" process and is discussed by Steinkamp (1995). In addition, a "disordered" component of the interpenetration can be expected to arise as a result of fluctuations away from the mean flow. The turbulence transport equations are capable of representing this "disordered" component of interpenetration. The two-field equations are capable of exhibiting the origin for these fluctuations. They are unstable whenever there is relative motion between the two fluids. Thus the two-field formulations contain hints of a disordered component to interpenetrating flow but require further modeling for practical exploitation of this feature. As usually written, two-field models are incapable of representing crucial aspects of the general turbulent dynamics that we seek to describe.

Directly descendent from the two-field models are those that have been extended to include the missing processes. These modifications are derived through empirical and heuristic arguments. They include

1. enhancement (by an order of magnitude with respect to a single sphere) of inter-field momentum exchange (drag) coefficients; 
2. incorporation of an evolution equation for the effective length scale, representing the effects of disperse phase clumping into larger and larger sizes during interpenetration;

3. the addition of a disordered component and the diffusive effects thereof, with energy source proportional to the loss that results from drag.

Another branch in the diagram of Fig. I.1 shows the development of single-point turbulence transport equations, also directly from the Navier-Stokes equations. These representations of the fine-scale fluctuational behavior of the fluid bear a close analogy to the descriptions of molecular dynamics expressed by the Navier-Stokes equations themselves. Just as the equations for gas dynamics are obtained from moments of a Boltzmann equation, so also the turbulence transport equations are formulated by means of moments of the Navier-Stokes equations. The parallel is illustrated in Fig. I.2

Much work has been directed over the last three or more decades towards development of the single-point turbulence transport branch for a single fluid with constant density. The two-point (spectral) branch, which represents the principal focus for this report, has its roots in the work of Heisenberg (1948), Kovasnay (1948), and Leith (1967, 1971), and more recently Cambon (1979), Cambon, Jeandel, and Mathieu (1981), Bertoglio (1982), Besnard et al. (1990), and Clark and Spitz (1995).

At the bottom of the chart of Fig. I.2 we show one of the ultimate products to which this research can be directed, the generalized single-point transport equations with the necessary additional features for realistic and practical problem solving over a wide scope of circumstances. Achievement of this goal requires the extensive consideration of issues related to the spectral structure of "turbulence," in it's most general sense:

- ordered and disordered components of the behavior;

- anisotropic structures in both physical space and wave-number space;

- self-similarity. 


\begin{tabular}{|c|c|c|}
\hline & Molecular Dynamics & Turbulence Transport \\
\hline A & $\begin{array}{l}\text { Many-particle distribution-function } \\
\text { transport, based on } d x_{i} / d t=u_{i} \text { and } \\
m d u_{i} / d t=F_{i}\end{array}$ & $\begin{array}{l}\text { The "exact equations for fluid dynam- } \\
\text { ics, the Navier-Stokes equations }\end{array}$ \\
\hline $\mathrm{B}$ & $\begin{array}{l}\text { BBGKY reduction to transport } \\
\text { equations for } \mathrm{N} \text {-particle distribution } \\
\text { functions involving integrals over } \\
\mathrm{N}+1 \text {-particle distribution } \\
\text { functions (exact but unclosed; but is } \\
\text { the process convergent?) }\end{array}$ & 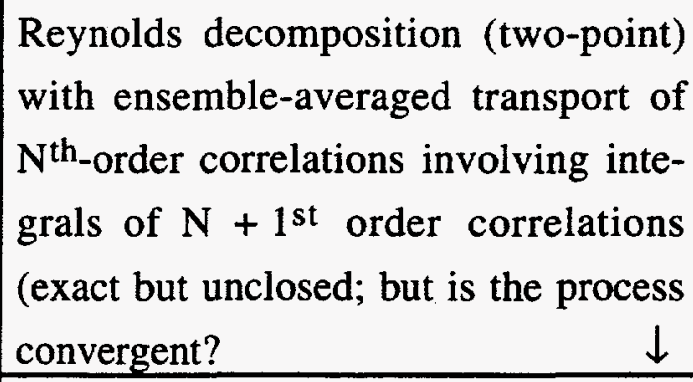 \\
\hline $\mathrm{C}$ & $\begin{array}{l}\text { "Chaotic" assumption; reduction to } \\
\text { Boltzmann equation } \\
\end{array}$ & $\begin{array}{lcc}\text { QN approximations plus closure } \\
\text { modeling techniques } & \downarrow \\
\end{array}$ \\
\hline $\mathrm{D}$ & $\begin{array}{l}\text { Single-relaxation-time model for } \\
\text { integral terms; often serves as a } \\
\text { good approximation for Boltzmann } \\
\text { equation } \\
\end{array}$ & $\begin{array}{l}\text { Spectrally local representations for cas- } \\
\text { cade, return to isotropy, and other } \\
\text { processes } \\
\downarrow\end{array}$ \\
\hline $\mathrm{E}$ & $\begin{array}{l}\text { Small-departures from equilibrium } \\
\text { to give distribution-function forms } \\
\text { for moment equations (Chapman- } \\
\text { Enskog for the stress tensor) lead- } \\
\text { ing to Navier-Stokes equations } \\
\text { (requires experimental determination } \\
\text { of transport functions) }\end{array}$ & $\begin{array}{l}\text { Locally self-similar form functions for } \\
\text { spectral moment integrals to give single- } \\
\text { point transport equations (e.g., } \mathrm{R}_{\mathrm{ij}} \varepsilon_{\mathrm{ij}} \text {; } \\
\mathrm{K}-\varepsilon \text {; BHR forms) (requires experimen- } \\
\text { tal determination of transport functions) } \\
\downarrow\end{array}$ \\
\hline $\mathrm{F}$ & $\begin{array}{l}\text { Becomes the starting point for the } \\
\text { right column }\end{array}$ & $\begin{array}{l}\text { Quasi-equilibrium approximations for } \\
\text { local point-functional representations } \\
\text { (e.g., mixing-length forms). } \\
\text { (Resembles closure modeling } \\
\text { techniques for gradient-flux expressions } \\
\text { and for approx. of triple-correlation } \\
\text { transport in EDQNM) }\end{array}$ \\
\hline
\end{tabular}

Fig. 1.2. Diagram showing the "family tree" for turbulence models. 


\section{THE TWO-FLUID SPECTRAL APPROACH}

For two or more interacting fluids, the usual approach to describing their interpenetration has been through the use of two-field or multifield equations, as described above. More novel is to consider the problem in terms of a single field of variables in which there are large variations in fluid density. (Single-point models have been described, and applied with considerable success, by Andronov, et al. (1982), by Nikiforov (1991), and by Besnard et al. (1987). Two-point models are only recently being investigated, notably by Clark and Spitz (1995), Godeford and Cambon (1994), for homogeneous circumstances, and in this current work for inhomogeneous configurations.

In this study we obtain correlations that are functions of two points in space. We pass to wave-vector space by means of Fourier transforms of the separation variable of the two points. Due to the complex nature of the transformed governing equations, we angularly integrate these equations in $\mathbf{k}$-space thus reducing the spectral dependence to $\mathbf{k} \mathbf{k}$. This transformation allows for a convenient identification of length scales to Fourier modes. We realize that this simplification captures only the real part and not the imaginary part of the spectrum and that a significant portion of the physics is averaged out of the equations. For now, however, we feel that a fully threedimensional $\mathbf{k}$-space model is unwarranted due to lack of any experimental verification.

In the current form of our representations, we are not especially concerned with that part of the spectrum that is associated with density discontinuities and is richly filled with the high-wavenumber components. Its existence requires consideration, possibly through boundary conditions at high-wave-numbers in k-space, but for our purposes the dominant spectral structure is associated with relatively large clouds or clumps of a fine-scale dispersed phase or with large-scale coalescent structures. Thus we ignore for now the interesting questions that can arise with a large Schmidt number, in which the $\mathrm{k}^{-1}$ "passive-scalar" behavior of imbedded material in a carrier fluid extends well beyond the wave numbers for which viscosity effects dominate the dynamics of the $\mathbf{R}_{\mathrm{ij}}$ spectrum.

Building on the analytical foundations of Clark and Spitz, we incorporate much guidance from two-field formulations and from many of the constant-density developments, both singlepoint and spectral. These relationships are discussed more fully in the next section, which describes the equations and the origins of contributing terms.

It will be noted that the equations for our current formulation have already been angularly averaged in (wave vector) $\mathbf{k}$-space so that the variables are functions of $\mathbf{k} \equiv|\mathbf{k}|$ (wave number as well as $\mathbf{x}$ and $t$ ). Moreover, for clarity of description we give them first as functions as $k, y, t$, only, as specialized to a free mixing layer of infinite extent in the $\mathrm{x}$ and $\mathrm{z}$ directions and accordingly the ensemble averages varying spatially only in the y direction. 
As with single-point turbulence transport modeling, there are many powerful constraints that useful spectral equations must include. Some of these are discussed in Sections III and IV. The list includes

- conservation of mass, momentum, and energy in both physical and wave-number space;

- $\quad$ proper tensor formulation;

- same free indices

- same symmetries

_ proper tensor properties: modeled terms in comparison with unmodeled terms;

- dimensional consistency;

- Galilean invariance;

- integrability (over wave numbers) to reasonable single-point forms;

- in absence of further guidance, simplicity, manifested by omission of certain products of first derivatives and of higher derivatives than second (in the spirit of the Stokes stress tensor in the Navier-Stokes equations);

- realizeability, more generally that the modeling obeys some (as yet unformulated) principal resembling the second law of thermodynamics.

These constraints help us to achieve meaningful formulations. They may lead to simple loworder approximations (e.g., for cascade in wave-number space) that capture much of the physics of what is happening, both qualitatively and quantitatively, as illustrated by the test examples described in this report.

To summarize the motivations for developing a tractable and broadly applicable spectral formulation for turbulence in a fluid with variable density, we have mentioned its relationship to the enhancement of single-point models. In Section I it was pointed out that a transport equation for the dissipation tensor must be derived. For this endeavor, the knowledge of the scales associated with the turbulence proves very useful for formulating credible turbulence transport equations. The spectral formulation described in this report provides a basis on which to extract scale information of the turbulence. In addition, the spectral studies go much further in delivering both theoretical and practical value.

- They serve as a basis for understanding many properties of turbulence, for example anisotropic self-similarity.

- They show the limitations of single-point models in circumstances of rapid transients.

- They remove the constraints of assumed spectral equilibrium in situations of spectral transients. 
- Frees the modeling from the simplistic characterization of turbulence by the magnitude of turbulence energy $(K)$ and a turbulent length scale $(L)$, or a dissipation rate $(\varepsilon)$.

- Leads to less drastic modeling assumptions.

- Not limited to certain regimes of Reynolds numbers.

- Hence, they allow a much greater scope of interesting problems to be solved realistically, albeit at greater computational expense, and allow the derivation of model equations that go beyond current one-point formulations in their applicability (as shown in Section IX). 


\section{FORMULATION OF THE MODEL EQUATIONS}

Development of the spectral model equations is described schematically in Fig. III.1. From the spectral formulation there radiate numerous directions for investigation as shown in Fig. III.2.

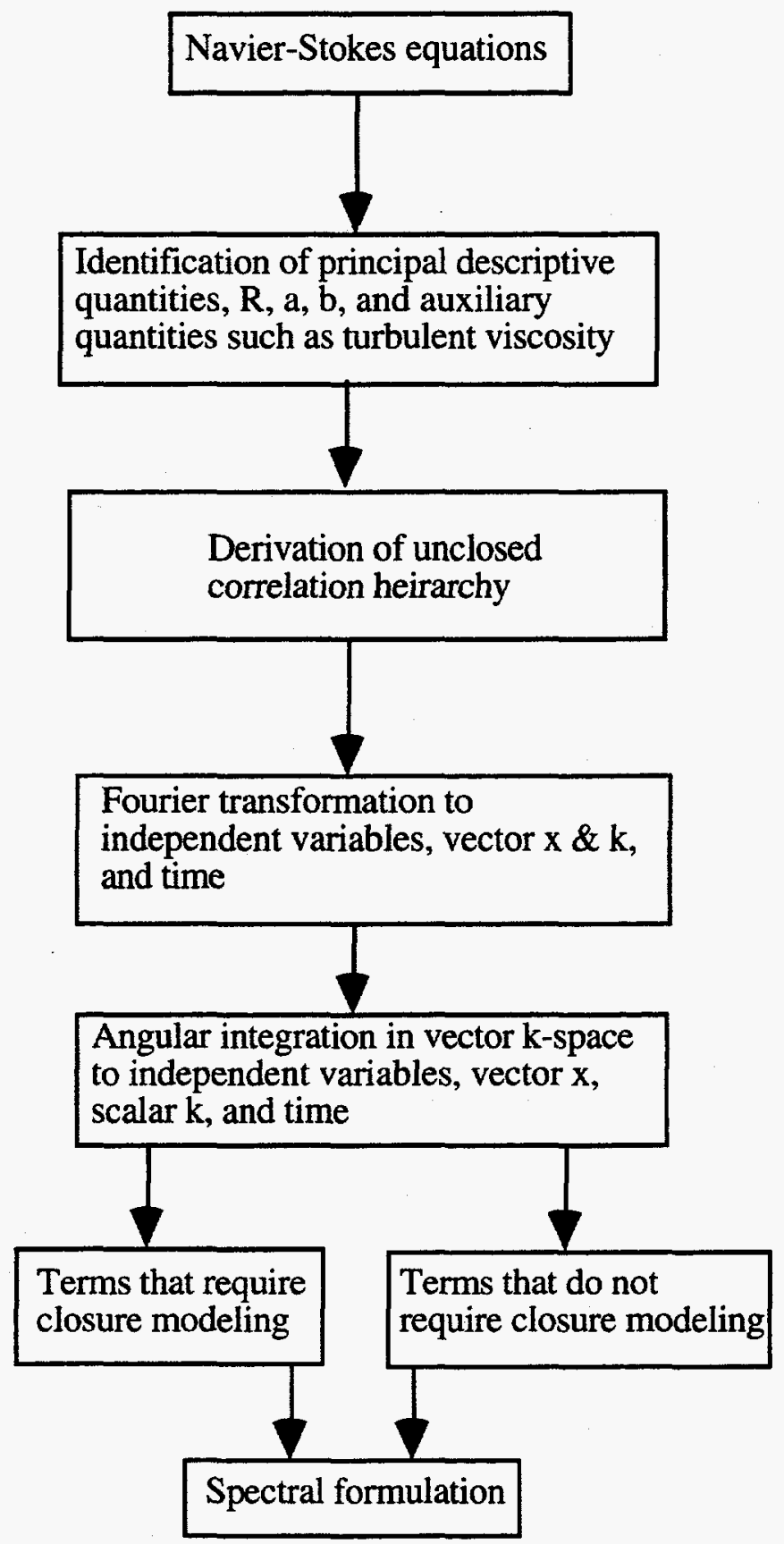

Fig. III.1. Flow chart describing steps of the spectral formulation. 


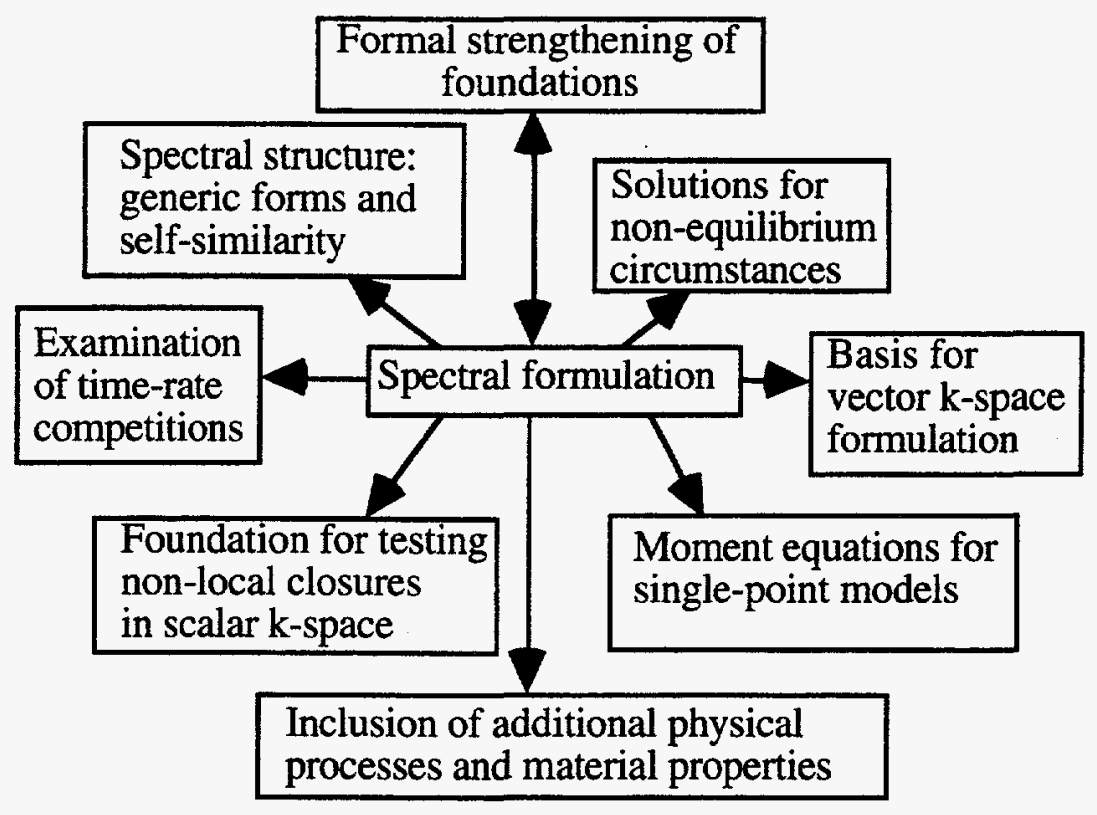

Fig. III.2. Flow chart describing all of the potential uses of a spectral formulation.

The physical processes described by the spectral transport equations are of three types, advective, pressure-related, and viscous. For each there are several features that need representation.

- Advective processes

- mean-flow advective transport

- mean-flow source to $R_{\mathrm{ij}}$

- stochastic advective transport

- kinematics of mixing

- Pressure processes

- local

- differential acceleration of fluids

- momentum exchange (drag)

- nonlocal

- in physical space: mean-flow coupling, diffusion

- in wave-number space: cascade, return to isotropy

- Viscous processes

- diffusion

- dissipation to heat

- momentum exchange between fluids 
The representation of these processes is described in the discussion of terms for each of the transport equations. Some of them descend directly from the Navier-Stokes equations without the need for closure modeling. Others require the derivation or postulate of closure modeling, for which some closure constraints were described in the previous section. As guidance for the development of closure modeling we further constrain our approach by means of identifying a one-to-one correspondence between turbulent processes and to the individually modeled terms. Although, for example, the processes of return to isotropy, diffusion, and cascade in wavenumber space all have their origins in pressure-velocity correlations, we employ separate terms for their effects with neither allowed to represent any part of the other. In this manner we intend to capture the dominant effects of each without the higher-order complexity of combined effects representation. Several other instances are mentioned in the descriptive text for each of the terms that have required modeling.

Wherever possible, closure modeling has been guided by extension of the procedures employed by previous investigators for single-point (nonspectral) closure.

With all of these constraints, both hard and soft, there remains surprisingly little flexibility in the formulation, so it should not be unexpected that applications to real-world circumstances exhibit a good degree of validity. The principal areas that remain for extension lie in nonlocal processes in wave-number space. These extensions for the variable-density formulation will likely result in additional tightening of agreement between calculations and experiments.

Although this discussion focuses on closure modeling by means of severely constrained postulates, there are several partially-derivational techniques that can be employed. Two examples are the following:

- Closures for an $\mathrm{N}^{\text {th }}$-order correlation are often accomplished through the derivation of its transport equation, the appending of a dissipation term proportional to the correlation, the neglect of both time variations and advection, and a very simple treatment of the $\mathrm{N}+1^{\text {st }}$-order terms. In this way the $\mathrm{N}^{\text {th }}$-order correlation emerges as a purely algebraic expression of the lower-order quantities. The famous Boussinesq approximation can be obtained in this fashion, and gradient-flux expressions for a variety of closures have likewise been derived.

- A useful closure guidance comes from the idea that the larger-scale part of the turbulence spectrum can be temporarily viewed as mean flow. Source terms for the small-scale part of the turbulence are then modeled from the "mean-flow" driving terms, and the loss from the large-scale parts is equated to the gain to the small-scale part. The next step is to return to the viewpoint to consider the entire structure of scales to be turbulence and thus to use the derived source-sink model as a representation of cascade flux through wave-number space. 


\section{THE SPECTRAL EQUATIONS}

Following the usual convention of single-point turbulence modeling of variable density flows, the flow field variables, $\rho, \mathbf{u}$, and $p$, are decomposed into their mean and fluctuating parts and substituted into the conservation equations. The decompositions are

and

$$
\begin{aligned}
& \rho=\bar{\rho}+\rho^{\prime}, \\
& \mathbf{u}=\overline{\mathbf{u}}+\mathbf{u}^{\prime}, \\
& \mathrm{p}=\overline{\mathrm{p}}+\mathrm{p}^{\prime},
\end{aligned}
$$

where the overbar denotes the non-mass-weighted ensemble average and the prime denotes a fluctuation about the average with the average of a fluctuating quantity equal to zero. For variable density flows, it is useful to incorporate the mass-weighted averaging procedure introduced by Favre that leads to a conservative form of the Reynolds stress tensor, $R_{i j}$, in the averaged momentum equations, where $R_{i j}(x, t)=\overline{\rho(x) u^{\prime \prime}(x) u^{\prime \prime}(x)}$. The averaged massweighted velocity, $\tilde{\mathbf{u}}$, is defined as

$$
\tilde{\mathbf{u}}=\frac{\overline{\rho \mathbf{u}}}{\bar{\rho}},
$$

and $\mathbf{u}$ " denotes the mass-weighted fluctuation about this averaged quantity, such that $\mathbf{u}=\tilde{\mathbf{u}}+\mathbf{u}^{\prime \prime}$ with $\overline{\rho u^{\prime \prime}}=0$.

There exists a strong motivation to identify a velocity associated with the average mass flux, $\overline{\mathrm{pu}}$, since this quantity occurs in both the averaged conservation of momentum equation as well as the averaged conservation of mass equation. If we apply the Reynolds decomposition to this momentum density, $\overline{\rho \mathbf{u}}$, we get

$$
\overline{\rho u_{i}}=\overline{\left(\bar{\rho}+\rho^{\prime}\right)\left(\bar{u}_{i}+u_{i}^{\prime}\right)}=\overline{\rho u_{i}}+\overline{\rho^{\prime} u_{i}^{\prime}} .
$$

In order to identify a velocity that is responsible for fluxing the mass, we can use the Favre mass weighted velocity, $\tilde{\mathbf{u}}$. Thus we incorporate the mass weighted velocity into the expression for momentum density to get

$$
\tilde{\mathrm{u}}_{\mathrm{i}}=\overline{\mathrm{u}}_{\mathrm{i}}+\frac{\overline{\rho^{\prime} \mathrm{u}_{\mathrm{i}}^{\prime}}}{\overline{\bar{\rho}}} .
$$


We define another important variable here, namely the velocity a, associated with the net mass flux relative to the $\tilde{\mathbf{u}}$ frame of reference. From the above equation, we can see that this velocity associated with this mass flux is just

$$
a_{i}=\frac{\overline{\rho^{\prime} u_{i}^{\prime}}}{\bar{\rho}} .
$$

This is a very important quantity as far as this study is concerned. It is this quantity that describes the fluxing of mass relative to the $\tilde{\mathbf{u}}$ frame of reference, and this idea is quantified by the resulting expression:

$$
\tilde{\mathbf{u}}=\overline{\mathbf{u}}+\mathbf{a}
$$

A transport equation for $\mathbf{a}$ is derived from the Navier-Stokes equations. This transport equation contains a source term, namely $b$, which couples with the mean pressure gradient, where $b=-\overline{\rho^{\prime}\left(\frac{1}{\rho}\right)^{\prime}}$. A transport equation for $b$ is derived from the conservation of mass equation.

Clark and Spitz (1995) give an extensive description of the foundations for two-point spectral representations of variable-density turbulence and describe the numerous challenges and issues associated with closure modeling for circumstances in which the distribution of one fluid throughout the other is purely homogeneous. Clark (personal notes, 1994) has subsequently proposed an extension for inhomogeneous distributions. The functions that he uses are similar to the single-point descriptors introduced in the BHR model. For two arbitrary points in space, $\mathbf{x}_{1}$ and $\mathbf{x}_{2}$, the two-point generalization of the single-point descriptors in the BHR model are

$$
\begin{gathered}
R_{i j}\left(x_{1}, x_{2}\right)=\frac{1}{2} \overline{\left[\rho\left(x_{1}\right)+\rho\left(x_{2}\right)\right] u_{i}^{\prime \prime}\left(x_{1}\right) u_{j}^{\prime \prime}\left(x_{2}\right)}, \\
a_{i}\left(x_{1}, x_{2}\right)=-\overline{u_{i}^{\prime \prime}\left(x_{1}\right) \rho\left(x_{1}\right) v\left(x_{2}\right)} \\
b\left(x_{1}, x_{2}\right)=-\overline{\rho^{\prime}\left(x_{1}\right) v^{\prime}\left(x_{2}\right)}
\end{gathered}
$$

where

$$
v(\mathbf{x})=\frac{1}{\rho(\mathbf{x})}
$$

Note that there are other forms that could be used for these quantities, which also satisfy the requirement of reducing to the single-point form as $\mathbf{x}_{1} \rightarrow \mathbf{x}_{2}$ and possess the desired symmetries, i.e., those possessed by the constant density Reynolds stress tensor.

The constraints on the Reynolds stress tensor, as required by Clark and Spitz (1995), are 
(1) $\quad R_{\mathrm{ij}}\left(\mathbf{x}_{1}, \mathrm{x}_{2}\right)=\mathrm{R}_{\mathrm{ji}}\left(\mathrm{x}_{2}, \mathrm{x}_{1}\right)$

(2) As $\left|\mathbf{x}_{1}-\mathbf{x}_{2}\right| \rightarrow \infty, \mathrm{R}_{\mathrm{ij}}\left(\mathbf{x}_{1}, \mathbf{x}_{2}\right) \rightarrow 0$

(3) $\quad \mathbf{R}_{\mathrm{ij}}\left(\mathbf{x}_{1}, \mathbf{x}_{2}\right)$ remain bounded as $\left|\mathbf{x}_{1}-\mathbf{x}_{2}\right| \rightarrow 0$.

There is no obvious constraint as to which choice is best except that whatever is chosen must be correctly transported by the Navier-Stokes equations. The choice is thus dictated more by properties of manipulative ease and transparency of interpretation.

Let us define

$$
\begin{gathered}
\mathbf{x} \equiv \frac{1}{2}\left(\mathbf{x}_{1}+\mathbf{x}_{2}\right), \\
\mathbf{r} \equiv \mathbf{x}_{1}-\mathbf{x}_{2}
\end{gathered}
$$

and substitute into the transported variables. We have for the additional Fourier transformations

$$
\begin{aligned}
R_{i j}(x, k) & \equiv \int R_{i j}(x, r) e^{-i k \cdot r} d r \\
a_{i}(x, k) & \equiv \int a_{i}(x, r) e^{-i k \cdot r} d r \\
b(x, k) & \equiv \int b(x, r) e^{-i k \cdot r} d r
\end{aligned}
$$

In this report, we work with variables and equations that have been angularly averaged in $\mathbf{k}$ space:

$$
\begin{gathered}
R_{i j}(x, k)=\int R_{i j}(x, k) \frac{k^{2} d \Omega_{k}}{(2 \pi)^{3}}, \\
a_{i}(x, k)=\int a_{i}(x, k) \frac{k^{2} d \Omega_{k}}{(2 \pi)^{3}}
\end{gathered}
$$

and,

$$
b(\mathbf{x}, k)=\int b(\mathbf{x}, \mathbf{k}) \frac{k^{2} d \Omega_{k}}{(2 \pi)^{3}}
$$

where $d \Omega_{k}=\sin \theta d \theta d \phi$ for $0 \leq \theta \leq \pi ; 0 \leq \phi \leq 2 \pi$. From these we can recover the single-point forms 


$$
\begin{aligned}
& R_{i j}(x)=\int_{0}^{\infty} R_{i j}(x, k) d k, \\
& a_{i}(x)=\int_{0}^{\infty} a_{i}(x, k) d k,
\end{aligned}
$$

and,

$$
b(x)=\int_{0}^{\infty} b(x, k) d k
$$

Clark and Spitz (1995) derive equations for a completely homogeneous configuration. For the inhomogeneous free mixing layer, we describe the necessary extensions in the most strippeddown form that we have found to give agreement with experiments. Thus we consider the special case of a mixing layer of infinite extent in the $\mathrm{x}$ and $\mathrm{z}$ directions, so that ensemble averages vary only with normal direction, $y$, scalar wave number, $k$, and time. For this configuration, the ensemble averages are equivalent to averages over each $\mathrm{x}-\mathrm{z}$ plane. The fluid is subjected to a body force, $\rho g$, directed in the $y$ direction. Below the mixing layer is a fluid with density $\rho_{1}$; above the layer is a different fluid with density $\rho_{2}$; for all of our calculations $\rho_{1}<\rho_{2}$, so that when $\mathrm{g}<0$ the configuration is unstable and mixing takes place. We also discuss the consequences of a reversal of the sign of $\mathbf{g}$ after the mixing layer has become well developed. The fluid is confined by stationary boundaries far above and below the turbulent mixing zone $(T M Z)$, so that there is nowhere any vertical volumetric flux and $\overline{\mathbf{u}}(y, t) \equiv 0$, so that $\tilde{\mathbf{u}}(\mathbf{y}, t) \equiv \mathbf{a}$.

The appropriate equations for $\bar{\rho}$ and $\tilde{\mathbf{u}}$ are the same as for the single-point formulation. The equations are presented here for one spatial dimension, with $\tilde{\mathrm{u}}$ and a representing the $\mathrm{y}$ component of the respective velocity vectors.

$$
\begin{gathered}
\frac{\partial \bar{\rho}}{\partial \mathrm{t}}+\frac{\partial \bar{\rho} \tilde{\mathrm{u}}}{\partial \mathrm{y}}=0 \\
\frac{\partial \bar{\rho} \tilde{\mathrm{u}}}{\partial \mathrm{t}}+\frac{\partial \bar{\rho} \tilde{\mathrm{u}} \tilde{\mathrm{u}}}{\partial \mathrm{y}}=-\frac{\partial \mathrm{p}}{\partial \mathrm{y}}-\frac{\partial \mathrm{R}_{\mathrm{yy}}}{\partial \mathrm{y}}+\bar{\rho} \mathrm{g} \\
\tilde{\mathrm{u}}=\mathrm{a} .
\end{gathered}
$$

The new equations for the spectral variables, $a(y, k, t), b(y, k, t), R_{n n}(y, k, t)$, and $R_{y y}(y, k, t)$ are presented in the following discussion, along with descriptions of the contributing terms in each. Assuming axisymmetry, so that $R_{x x}=R_{z Z}$, the values for these quantities can be determined by knowing $R_{y y}$ and $R_{n n}$. For this configuration in principal coordinates, $R_{i j}$ has no off-diagonal 
components. With the addition of horizontal shear the additional terms are the same as those given by Besnard et al. (1990) in their analysis of a free shear, and equations for $R_{x y}$ and/or $R_{z y}$ are obtained as straightforward extensions.

For $a(y, k, t)$, we write

$$
\begin{aligned}
\frac{\partial \mathrm{a}}{\partial \mathrm{t}}= & -\tilde{\mathrm{u}} \frac{\partial \mathrm{a}}{\partial \mathrm{y}}+\frac{\mathrm{b}}{\bar{\rho}} \frac{\partial \overline{\mathrm{p}}}{\partial \mathrm{y}}-\left[\mathrm{C}_{\mathrm{rp} 1} \mathrm{k}^{2} \sqrt{\mathrm{a}_{\mathrm{n}} \mathrm{a}_{\mathrm{n}}}+\mathrm{C}_{\mathrm{rp} 2} \mathrm{k} \sqrt{\frac{\mathrm{kR} \mathrm{nn}}{\bar{\rho}}}\right] \mathrm{a}-\frac{\mathrm{R}_{\mathrm{yy}}}{\bar{\rho}^{2}} \frac{\partial \bar{\rho}}{\partial \mathrm{y}} \\
& +\frac{\partial}{\partial \mathrm{k}}\left\{\mathrm{k}^{2} \sqrt{\frac{\mathrm{kR} \mathrm{nn}}{\bar{\rho}}}\left[-\mathrm{C}_{1} \mathrm{a}+\mathrm{C}_{2} \mathrm{k} \frac{\partial \mathrm{a}}{\partial \mathrm{k}}\right]\right\}+\mathrm{C}_{\mathrm{d}} \frac{\partial}{\partial \mathrm{y}} v_{\mathrm{t}} \frac{\partial \mathrm{a}}{\partial \mathrm{y}} .
\end{aligned}
$$

The contributing terms, discussed in the order they appear on the right side, are

1. the advective term relative to the volume-weighted coordinate system.

2. a principal driving term, written as a direct extension to the term in single-point formulation, on the assumption that spectral $b$ produces spectral $a$ at the same wave number. From direct numerical simulation calculations, there is reason to believe (Sandoval, 1995) that there are modifications to this term that arise from inclusion of appropriate modeling of the closure for a single-point term like

$$
\overline{\bar{\rho}\left(\frac{1}{\rho}\right) \frac{\partial p^{\prime}}{\partial x_{i}}}
$$

The effect can be described by using some appropriate fraction of $b(k)$ in this source term; we have not, however, included this modification in our present investigations. The assumption that $b(k)$ produces $a_{i}(k)$ at the same $k$ is somewhat justified by the fact that Clark and Spitz (1995) apply an approximate Rapid Distortion Theory to their model for the case of homogeneity and find that $\frac{\partial a(k)}{\partial t}=b(k) \frac{\partial \bar{p}}{\partial y}$. Sandoval (1995) has also given reasons to believe that this is true from his DNS calculations of homogeneous variable density turbulence. Note that this term contributes a pressure gradient to the equation $\tilde{\mathbf{u}}=\mathbf{a}(\mathrm{y}, \mathrm{t})$, which serves to determine the value of the pressure gradient at every time through the transient dynamics.

3. the representation of drag between the two fluids. The $\mathrm{C}_{\mathrm{rp} 1}$ term represents the form drag, with $C_{\mathrm{rp} 1}$ being directly related to the drag coefficient that Youngs (1992) required to be an order of magnitude greater than the classical value for an isolated sphere. We confirm the Youngs finding and attribute the necessity for large magnitude to the effects of convoluted flow paths along the interface between the 
fluids. The $\mathrm{C}_{\mathrm{rp} 2}$ term represents the viscous drag, in this case resulting from effective turbulence viscosity rather than molecular viscosity. These terms are derived directly as extensions of the usual two-field formulation. There are some possible alternative expressions for these drag terms, which could incorporate relevant effects that are nonlocal in wave number space. For example, we could consider replacing

by

$$
-C_{\mathrm{rp} 1} k^{2} \sqrt{a_{n} a_{n}}
$$

$$
-C_{\text {Tp1 }} \int_{0}^{k_{1}} k \sqrt{a_{n} a_{n}} d k \text {. }
$$

In addition, these terms could depend on a nontrivial fashion on dimensionless functions of dimensionless $b(y, t)$. This nonlocal form describes contributions to drag at wave number $\mathrm{k}$ from all other wave numbers lying between $\mathrm{k}=0$ and $\mathrm{k}=\mathrm{k}_{1}$. There is no difficulty with this formulation if we attempt to let $k_{1} \rightarrow \infty$. With the expectation that $a \rightarrow k^{-7 / 3}$ for large $k$ (see form-function discussion below), the integral converges in that limit. Similar comments can be made about possible nonlocal forms for the $\mathrm{C}_{\mathrm{rp} 2}$ term. It is tempting to consider various nonlocal drag expressions because of the heuristic idea that the effects of many wave numbers can combine collectively to transfer momentum at any particular wave number between the fluids. We have not at this stage found any formulation that works better than the simple local form, insofar as comparisons with experimental data are concerned.

4. a second principal drive term. This is called the gradient-flux term because whenever it is roughly balanced by drag the result is an expression for $\bar{\rho} \mathbf{a}$ (the spectral mass flux), proportional to the gradient of $\bar{\rho}$. Term $\# 2$, in contrast, can contribute a counter-gradient flux, notably when reversal of gravity turns the mixing zone into a demixing zone. More generally, the two principal drive terms, \#2 and \#4, interact with the other terms in the a equation in various possible ways. In the start-up phase of unstable mixing, the pressure-gradient term acts wherever $b$ is seeded at the interface to drive a strongly-ordered part of $\partial \mathrm{a} / \partial \mathrm{t}$. The growing value of a interacts with the pressure gradient in the $R_{n n}$ and $R_{y y}$ equations (see below), which produces a disordered component of Reynolds stress, giving in turn a source to a disordered component of $\partial \mathrm{a} / \partial \mathrm{t}$. In this mixing stage of the TMZ growth, the two sources reinforce each other. As the TMZ dynamics mature into self-similar growth, the large-wave-number components of $b$ may cascade into an inactive range, which means that for those wave numbers the pressure-gradient source to $\partial \mathrm{a} / \partial \mathrm{t}$ has changed 
to essentially complete balance with the drag term. Only the low-wave-number parts of $b$ (along with the density-gradient source) are effective in perpetuating the continuing self-similar growth of $a$. The spectrum of $b$ can be considered to possess active and passive parts, which is significant for deriving single-point equations from the spectral equations. More details regarding the concept of active and passive parts of the $b(k)$ spectrum are given in Section VII.C in the discussion of acceleration reversal. If $\mathbf{g}$ suddenly vanishes, then only the \#4 source term remains to continue widening the TMZ; however, its effectiveness for this purpose is significantly curtailed by the drag (\#3) and cascade (\#5) terms. When $\mathbf{g}$ is completely reversed, we have an adjustment period in which the \#2 source term works in concert with drag (\#3), across all parts (previously active and passive) of the b spectrum, to accomplish the reversal of a. This represents a spectral nonequilibrium process. Only when a has changed sign do these terms again oppose each other and the passive part of the $b$ spectrum returns to dormancy. It is interesting to confirm in calculations of these processes that the passive part of the $b$ spectrum indeed has very little effect on the overall spectrally integrated dynamics, as demonstrated by comparison of two calculations, one that retains the passive part and the other that discards it. The only significant difference between the two calculations lies in the value of b; everything else is essentially the same.

5. the cascade terms. These are both conservative in k-space. They are based on the model proposed by Leith (1967) for local cascade, with a wave-like part (the $C_{1}$ term) and a diffusive part (the $C_{2}$ term); see Besnard et al. (1990) for an extensive discussion. With $\mathrm{C}_{1}>0$ the wave-like cascade is direct (i.e., to higher wave numbers). Of necessity, $\mathrm{C}_{2}>0$, resulting in both direct and inverse contributions to cascade. Several nonlocal forms of cascade representation have been considered. These nonlocal forms attempt to represent the triad interactions associated with triplecorrelation terms in the formal derivation for constant-density turbulence as integrals over k-space (e.g., EDQNM or DIA derivations). Integral formulations for the variable density case are not yet rigorously developed, but one way to accomplish some degree of nonlocality would come (as in the drag term \#3) from replacement of the local cascade rates by nonlocal integral cascade rates, for example

becomes

$$
\mathrm{k} \sqrt{\frac{\mathrm{kR}_{\mathrm{nn}}(\mathrm{k})}{\bar{\rho}}}
$$




$$
\sqrt{\int_{0}^{k_{1}} k^{2} \frac{R_{n n}(k)}{\bar{\rho}} d k} .
$$

Both forms are essentially equivalent in the inertial range. It should be noted that $R_{n n}(k)$ is not a nonnegative definite function; hence the simpler local form (IV.22) will fail for circumstances of $R_{n n}(k)<0$. Clark and Spitz (1995) chose form (IV.23) on this basis and also on the observation that the so-called "catastrophe time" (Leisure, 1990) for constant-density isotropic turbulence is predicted with good agreement to EDQNM. However, for most circumstances, we have seen little difference between the results using either cascade rate time-scale, and we have not encountered negative values of $R_{n n}(k)$; thus we have opted for the simpler form (IV.22). Indeed, it appears, as stated above, that the modeling constraints (conservation, tensor form, dimensionality, etc.) used for our cascade (and other) terms enable the simplest forms (like term \#5) to capture an enormous portion of the physical processes that we wish to represent.

6. spatial diffusion. This term is the same as used by many previous authors and requires two comments pertaining to the possible choices for expressions for $v_{t}$ : first, it is well known that the eddy viscosity, $v_{t}$, should be modeled nonlocally; and second, the processes associated with an eddy viscosity are anisotropic. Nevertheless, until further investigation, for simplicity we chose an isotropic form which reduces to the commonly used single point form

$$
v_{t}=0.09 S \sqrt{K},
$$

in which $\mathrm{S}$ is the mean turbulent scale and $\mathrm{K}$ is the total turbulence energy per unit mass. To accomplish this correspondence requires the identification of a generic form function for $R_{n n}$ and the performance of an appropriate moment integral, as discussed below. An appropriate $C_{D}$ must be chosen.

Next, for $b(y, k, t)$ we write

$$
\begin{aligned}
\frac{\partial b}{\partial t} & =\left(\frac{2 \bar{\rho}-\rho_{1}-\rho_{2}}{\rho_{1} \rho_{2}}\right) \frac{\partial \bar{\rho} a}{\partial y}-C_{f b}\left[\bar{v}^{2} \frac{\partial}{\partial y}\left(\frac{\bar{\rho}}{\bar{v}}\right)\right] \frac{\partial k a}{\partial k} \\
& +\frac{\partial}{\partial k}\left\{k^{2} \sqrt{\frac{k R_{n n}}{\bar{\rho}}}\left[-C_{1} b+C_{2} k \frac{\partial b}{\partial k}\right]\right\}+C_{d} \frac{\partial}{\partial y} v_{t} \frac{\partial b}{\partial y}-C_{d b} k^{2} D b .
\end{aligned}
$$


The contributing terms on the right side are

1. the kinematical source term, derived by Steinkamp (1995) for single-point transport of $b$. Because this term describes the effects of fluid mass transfer, there is no requirement for an advective term in the $b$ equation. This term maintains the value of $b$ at nearly its configurational value, $\alpha_{1} \alpha_{2}\left(\rho_{1}-\rho_{2}\right)^{2} / \rho_{1} \rho_{2}$.

2. the transport of $b$ through wave-number space, as induced by the presence of inhomogeneity in the mixture of fluids. This term was formally derived by Clark (personal notes, 1994) as a next-higher-order contribution to the Taylor expansion of points, $x_{1}$ and $x_{2}$ about the central point, $x$. It resembles the $C_{f}$ terms previously proposed by Besnard et al. (1990) to describe the mean-flow-shear-induced distortions of turbulence spectra in a constant-density fluid. In that case, the term contributes to the vortex-pairing process that occurs in a free shear layer. Here it contributes to the bubble-doubling process that is known to occur in the self-similar stages of TMZ growth at small wave numbers and to an alteration of cascade that occurs through "eddy distortion" at high wave numbers. Its presence is very important to the achievement of agreement with experiments. A higher-order degree of nonlocality (e.g., an integral expression) may be appropriate, but at this stage there is no proof of the necessity for this complication.

A heuristic derivation of this term suggests that it must couple the inhomogeneity as described by $(1 / \bar{\rho}) \nabla \bar{\rho}$, with the presence of interpenetration, described by a. [Direct coupling to $(1 / \bar{\rho}) \nabla \bar{p}$ seems implausible, as this contributes to the creation of interpenetration and is not a measure of its current level.] Dimensional arguments restrict the possibilities appreciably; adding the necessity for conservation in k-space leads to a form proportional to

$$
\frac{1}{\bar{\rho}} \frac{\partial \bar{\rho}}{\partial \mathrm{y}} \frac{\partial \mathrm{ka}}{\partial \mathrm{k}}
$$

which is very close to the form derived by Clark (personal notes, 1994), and becomes identical to that term for small differences in density.

3. the cascade terms. These have the same forms as the cascade terms for $\mathbf{a}$, and the same comments apply here. It should be noted that cascade rates proportional to $\mathrm{k}^{2} \sqrt{\mathrm{ka}_{\mathrm{n}} \mathrm{a}_{\mathrm{n}}}$ could be proposed on dimensional grounds, although their physical significance is not obvious and their inclusion in calculations produces very minor effects, neither helpful nor harmful in matching results with existing experimental data. Similar to the discussion of (\#5) for the a equation, this expression is not a 
nonnegative definite function, and a similar argument is used for our choice of cascade rates.

4. the spatial diffusion term.

5. the decay term resulting from molecular diffusion between species, for which the kinematic diffusion coefficient is $D$. In the studies described here, $D \rightarrow 0$.

Next, for $R_{n n}(y, k, t)$ and $R_{y y}(y, k, t)$ we write

$$
\begin{aligned}
& \frac{\partial \mathrm{R}_{\mathrm{nn}}}{\partial \mathrm{t}}=-\frac{\partial \mathrm{R}_{\mathrm{nn}} \tilde{\mathrm{u}}}{\partial \mathrm{y}}+\int_{-\infty}^{+\infty}\left(2 \mathrm{a}\left(\mathrm{y}^{\prime}\right) \frac{\partial \mathrm{p}\left(\mathrm{y}^{\prime}\right)}{\partial \mathrm{y}^{\prime}}\right)\left(\frac{\exp \left(-2 \mathrm{k}\left|\mathrm{y}^{\prime}-\mathrm{y}\right|\right)}{\int_{-\infty}^{+\infty} \exp \left(-2 \mathrm{k}\left|\mathrm{y}^{\prime \prime}-\mathrm{y}\right|\right) \mathrm{dy^{ \prime \prime }}}\right) d \mathrm{y}^{\prime}-2 \mathrm{R}_{\mathrm{yy}} \frac{\partial \tilde{\mathrm{u}}}{\partial \mathrm{y}} \\
& +\mathrm{C}_{\mathrm{d}} \frac{\partial}{\partial \mathrm{y}} v_{\mathrm{t}} \frac{\partial \mathrm{R}_{\mathrm{nn}}}{\partial \mathrm{y}}+\frac{\partial}{\partial \mathrm{k}}\left\{\mathrm{k}^{2} \sqrt{\frac{\mathrm{kR}_{\mathrm{nn}}}{\bar{\rho}}}\left[-\mathrm{C}_{1} \mathrm{R}_{\mathrm{nn}}+\mathrm{C}_{2} \mathrm{k} \frac{\partial \mathrm{R}_{\mathrm{nn}}}{\partial \mathrm{k}}\right]\right\},
\end{aligned}
$$

and

$$
\begin{aligned}
\frac{\partial \mathrm{R}_{\mathrm{yy}}}{\partial \mathrm{t}}= & -\frac{\partial \mathrm{R}_{\mathrm{yy}} \tilde{\mathrm{u}}}{\partial \mathrm{y}}+\int_{-\infty}^{+\infty}\left(2 \mathrm{a}\left(\mathrm{y}^{\prime}\right) \frac{\partial \overline{\mathrm{p}}\left(\mathrm{y}^{\prime}\right)}{\partial \mathrm{y}^{\prime}}\right)\left(\frac{\exp \left(-2 \mathrm{k}\left|\mathrm{y}^{\prime}-\mathrm{y}\right|\right)}{\int_{-\infty}^{+\infty} \exp \left(-2 \mathrm{k}\left|\mathrm{y}^{\prime \prime}-\mathrm{y}\right|\right) \mathrm{dy^{ \prime \prime }}}\right) \mathrm{dy^{ \prime }}-2 \mathrm{R}_{\mathrm{yy}} \frac{\partial \tilde{\mathrm{u}}}{\partial \mathrm{y}}+\mathrm{C}_{\mathrm{d}} \frac{\partial}{\partial \mathrm{y}} v_{\mathrm{t}} \frac{\partial \mathrm{R}_{\mathrm{yy}}}{\partial \mathrm{y}} \\
& +\frac{\partial}{\partial \mathrm{k}}\left\{\mathrm{k}^{2} \sqrt{\frac{\mathrm{kR} \mathrm{nn}}{\bar{\rho}}}\left[-\mathrm{C}_{1} \mathrm{R}_{\mathrm{yy}}+\mathrm{C}_{2} \mathrm{k} \frac{\partial \mathrm{R}_{\mathrm{yy}}}{\partial \mathrm{k}}\right]\right\}+\mathrm{C}_{\mathrm{m}} \int_{0}^{\mathrm{k}} \sqrt{\frac{\mathrm{kR} \mathrm{nn}}{\bar{\rho}} \mathrm{dk}}\left(\frac{1}{3} \mathrm{R}_{\mathrm{nn}}-\mathrm{R}_{\mathrm{yy}}\right) . \text { (IV.28) }
\end{aligned}
$$

The contributing terms on the right side are

1. the advective term. In contrast to the a equation, in which the conservation of mass equation is used to remove $\bar{\rho}$ from

$$
\frac{\partial \bar{\rho} \mathrm{a}}{\partial \mathrm{t}}+\frac{\partial \bar{\rho} \mathrm{a} \tilde{u}_{\mathrm{n}}}{\partial \mathrm{x}_{\mathrm{n}}}
$$

these similar terms in the equations for $R_{i j}$ retain the $\bar{\rho}$ that is intrinsically present in the generalized expression for the Reynolds stress.

2. a principal driving term, coupling a with the pressure gradient. This process is intrinsically nonlocal in physical space, with effects that reach progressively further 
away for wave numbers approaching zero (i.e., for large scales). The basis for this nonlocality lies in the propagation of pressure waves. In linear Kelvin-Helmholtz or Rayleigh-Taylor stability analysis the effects are manifested in a velocity potential that varies as $\exp [-\mathrm{k}|\mathrm{y}|]$, where $|\mathrm{y}|$ is the distance from the center of the instability layer. Thus the spreading of $R_{i j}$, which varies as the square of velocity fluctuations, is represented by the factor $\exp [-2 \mathrm{klyl}]$, as shown in the term. Otherwise the coupling of a with the pressure gradient is the same as the single-point coupling. The basis and consequences of this nonlocality spreading in physical space are discussed further in Section V.

3. another principal driving term, coupling $\mathrm{R}_{\mathrm{yy}}$ to gradients of $\tilde{\mathrm{u}}$. This is a standard term (Besnard et al. 1990) that is well known especially for constant-density turbulent flows, but which is equally relevant here (although of relatively minor importance for the TMZ studies).

4. the spatial diffusion term. As in the $\mathbf{a}$ and $b$ equations, the form to be used can be local or any of several nonlocal variants. As for the $\mathbf{a}$ and $b$ equations we use

$$
v_{t}=\int_{0}^{\infty} \sqrt{\frac{k R_{n n}}{\bar{\rho}}} \frac{d k}{k^{2}} .
$$

5. the cascade terms. The same formulation and comments apply here as for the cascade of $\mathbf{a}$.

6. the return-to-isotropy term, which appears only in the equation for $\mathbf{R}_{\mathbf{y y}}$. The return rate can be written either in local form or, as shown, in the nonlocal form

$$
C_{m} \int_{0}^{k} \sqrt{\frac{k^{\prime} R_{n n}}{\bar{\rho}}} d k^{\prime} .
$$

Another nonlocal form proposed by Herring (personal communication, 1994) addresses the issues discussed in term $\# 5$ of the a equation and is written as

$$
C_{m} \sqrt{\int_{0}^{k}\left(k^{\prime}\right)^{2} \frac{R_{n n}}{\bar{\rho}} d k^{\prime}} .
$$

We have tried both forms for the return-to-isotropy rate and have found negligible difference.

Boundary conditions must be considered whenever a finite-difference calculation is performed for limited domains in $\mathrm{k}$ and $\mathrm{y}$. In the $\mathrm{y}$ direction the domain simply extends well into 
the pure material on either side of the TMZ, where everything is at rest. In k-space we assume that the resolved part of the calculation is bounded by exponential behaviors for both the large and small wave numbers beyond the resolved region. This prescription allows the variable to flux through the boundaries. We also experiment with an exception to this procedure, in which the flux of $b$ at large wave numbers was set equal to zero, corresponding to $D=0$. As illustrated and discussed in the section on numerical results, Section VII, the effect on b is large; but the difference is almost entirely due to the retention or discarding of the passive parts of the $b$ spectrum, so that the effects on all the other quantities is negligible.

Values for the $\mathrm{C}$ coefficients in these equations have been chosen to be the same as those used by previous investigators for constant-density or single-point studies. For the drag coefficients in the a equation, we use values that are greatly enhanced (Youngs 1992) over those for isolated spheres, as discussed earlier in this section.

$$
\begin{aligned}
& C_{r p 1}=5.0 \\
& C_{r p 2}=6.0
\end{aligned}
$$

For the local cascade coefficients, we use the values discussed by Clark (1992).

$$
\begin{aligned}
& C_{1}=0 . \overline{12} \\
& C_{2}=0 . \overline{06}
\end{aligned}
$$

Spatial diffusion is modulated by the coefficient,

$$
C_{d}=0.03
$$

while

$$
\begin{aligned}
& C_{b d}=0.0 \\
& C_{m}=1.0
\end{aligned}
$$

and

$$
\mathrm{C}_{\mathrm{fb}}=0.5
$$

Because $\mathrm{C}_{\mathrm{fb}}$ is the one coefficient that is not ascertained in terms of previous investigations, we varied its value to determine the effect and observed that it is noticeable but not profound, as described below. Clark (1992) discusses a value of $C_{m}$ for constant density turbulence that is smaller than the value used here. We have tested the model for values of $C_{m}$ between that suggested by Clark $\left(C_{m}=0.17\right)$ and the value we use here and found negligible differences in the results. We suspect that this is due to the fact that our inhomogeneous flow is strongly dominated by the sources due to mixing that renders the return to isotropy, a second-order effect. 
For reference, we also give the full tensor forms of our base-model equations. In the reference frame with $\overline{\mathbf{u}} \equiv 0$,

$$
\begin{aligned}
& \frac{\partial a_{i}}{\partial t}=-\tilde{u}_{n} \frac{\partial a_{i}}{\partial x_{n}}+\frac{b}{\bar{\rho}} \frac{\partial \bar{p}}{\partial x_{i}}-\left[C_{r p 1} k^{2} \sqrt{a_{n} a_{n}}+C_{r p 2} k \sqrt{\frac{k R_{n n}}{\bar{\rho}}}\right] a_{i}-\frac{R_{i n}}{\bar{\rho}^{2}} \frac{\partial \bar{\rho}}{\partial x_{n}} \\
& +\frac{\partial}{\partial k}\left\{k^{2} \sqrt{\frac{k R_{n n}}{\bar{\rho}}}\left[-C_{1} a_{i}+C_{2} k \frac{\partial a_{i}}{\partial k}\right]\right\}+\frac{C_{d}}{\bar{\rho}} \frac{\partial}{\partial x_{n}} v_{t} \bar{\rho} \frac{\partial a_{i}}{\partial x_{n}} \\
& \frac{\partial b}{\partial t}=\left(\frac{2 \bar{\rho}-\rho_{1}-\rho_{2}}{\rho_{1} \rho_{2}}\right) \frac{\partial \bar{\rho} a_{n}}{\partial x_{n}}-C_{f b}\left[\bar{v}^{2} \frac{\partial}{\partial x_{n}}\left(\frac{\bar{\rho}}{\bar{v}}\right)\right] \frac{\partial \mathrm{ka}_{n}}{\partial k} \\
& +\frac{\partial}{\partial k}\left\{k^{2} \sqrt{\frac{k R_{n n}}{\bar{\rho}}}\left[-C_{1} b+C_{2} k \frac{\partial b}{\partial k}\right]\right\}+C_{d} \frac{\partial}{\partial y} v_{t} \frac{\partial b}{\partial y} \\
& \frac{\partial R_{i j}}{\partial t}=-\frac{\partial R_{i j} \tilde{u}_{n}}{\partial x_{n}}+\int_{-\infty}^{+\infty}\left[\left(a_{i}\left(y^{\prime}\right) \frac{\partial \bar{p}\left(y^{\prime}\right)}{\partial x_{j}}\right)+\left(a_{j}\left(y^{\prime}\right) \frac{\partial \bar{p}\left(y^{\prime}\right)}{\partial x_{i}}\right)\right]\left(\frac{\exp \left(-2 k\left|y^{\prime}-y\right|\right)}{\int_{-\infty}^{+\infty} \exp \left(-2 k\left|y^{\prime \prime}-y\right|\right) d y^{\prime \prime}}\right) d y^{\prime} \\
& -R_{i n} \frac{\partial \tilde{u}_{j}}{\partial x_{n}}-R_{j n} \frac{\partial \tilde{u}_{i}}{\partial x_{n}}+\frac{\partial}{\partial k}\left\{k^{2} \sqrt{\frac{k R_{n n}}{\bar{\rho}}}\left[-C_{1} R_{i j}+C_{2} k \frac{\partial R_{i j}}{\partial k}\right]\right\} \\
& +C_{m} \int_{0}^{k} \sqrt{\frac{k R_{n n}}{\bar{\rho}}} d k\left(\frac{1}{3} R_{n n}-R_{i j}\right)+C_{d} \frac{\partial}{\partial x_{n}} v_{t} \frac{\partial R_{i j}}{\partial x_{n}} .
\end{aligned}
$$




\section{NONLOCAL PROCESSES IN PHYSICAL SPACE}

Integral formulations in physical space enable the characterization of instantaneous pressurewave propagation of fluctuations from one point in physical space to another. Thus, for example, the existence of mean-flow shear can contribute to the nonlocal creation of turbulence at localities lying outside the shear layer. Likewise a TMZ between two fluids, subjected to a mean-field pressure gradient, can manifest the effects of differential acceleration beyond the borders of the zone.

Classical Rayleigh-Taylor analysis for the linear instability of an interface introduces a velocity potential that varies as $\exp (-\mathrm{k}|\mathrm{y}|)$, in which $\mid \mathrm{yl}$ is the distance from the interface (Chandrasekhar, 1961). If we assume that the amplitude of the perturbation on the interface is approximately half the width of the TMZ, then for a TMZ with width $\mathrm{W}$ and for wave numbers such that $\mathrm{kW}>1.0$, the linear theory does not apply. For parts of the turbulence spectrum with $\mathrm{kW} \ll 1.0$, however, the linear theory is relevant and shows that fluctuating components of velocity extend appreciably beyond the boundaries of the TMZ. This "creation-at-a-distance" of velocity fluctuations translates into a nonlocal source for $R_{i j}$. It does not, however, indicate nonlocal sources for $a_{i}$ or $b$, which are associated with the transport of fluid rather than pressure effects. We don't use, however, nonlocal sources for $a_{i}$ and $b$. For $b$, there are no pressure terms to provide this effect; for $a_{i}$, preliminary simulations of Sandoval (1995) indicate fluctuating pressure correlations act to modify the $b \frac{\partial \bar{p}}{\partial y}$, which is already included as a local term.

Guided by the results of linear analysis, we thus have modified the source terms for $R_{n n}$ and $R_{y y}$ in the following manner. The local form,

$$
\frac{\partial \mathrm{R}_{\mathrm{nn}}}{\partial \mathrm{t}}=2 \mathrm{a} \frac{\partial \overline{\mathrm{p}}}{\partial \mathrm{y}},
$$

is rewritten

$$
\frac{\partial R_{\mathrm{nn}}}{\partial \mathrm{t}}=\int_{-\infty}^{+\infty}\left[2 \mathrm{a} \frac{\partial \overline{\mathrm{p}}}{\partial \mathrm{y}}\right] \mathrm{Q}\left(\mathrm{y}^{\prime}, \mathrm{y}\right) \mathrm{dy^{ \prime }}
$$

and similarly for $\mathbf{R}_{\mathbf{y y}}$.

The kernel Q(y'y), "nonlocality function," must satisfy the condition

$$
\int_{-\infty}^{+\infty} Q\left(y^{\prime}, y\right) d y^{\prime}=1
$$


For our problem we also expect it to depend only on $y^{\prime}-\mathrm{y}$ and to decrease as exp[-2kly'-yl] as the separation between points increases. For large values of $\mathrm{k}, \mathrm{Q}\left(\mathrm{y}^{\prime}, \mathrm{y}\right)$ approaches a delta function.

For now, we choose

$$
\mathrm{Q}\left(\mathrm{y}^{\prime}, \mathrm{y}\right)=\mathrm{k} \exp \left[-2 \mathrm{k}\left|\mathrm{y}^{\prime}-\mathrm{y}\right|\right]
$$

which satisfies the normalization condition, behaves in the required manner as $\mathrm{k} \rightarrow 0$, and has the desired delta-function behavior as $\mathrm{k} \rightarrow \infty$. It remains to be demonstrated, however, that this "spreading" factor allows the turbulence model to recover the linear Rayleigh-Taylor behavior as $k \rightarrow 0$. We will assume that the configuration starts at rest with only $b$ present; the lowest order contribution to the evolution of a and $R_{i j}$ (when both are still very small) are described by the following subset of Eqs. (IV.28) and (IV.19). Consider the following parts at first without the $\mathrm{Q}\left(\mathrm{y}^{\prime}, \mathrm{y}\right)$.

$$
\begin{gathered}
\frac{\partial R_{y y}}{\partial t}=2 a \frac{\partial \bar{p}}{\partial y} \\
\frac{\partial a}{\partial t}=\frac{b}{\bar{\rho}} \frac{\partial \bar{p}}{\partial y}-\frac{R_{y y}}{\bar{\rho}^{2}} \frac{\partial \bar{\rho}}{\partial y}
\end{gathered}
$$

Differentiate the $R_{y y}$ equation with respect to $t$, allowing only for the variation of $\mathbf{a}$, and insert $\partial \mathrm{a} / \partial \mathrm{t}$ from the second equation.

$$
\frac{\partial^{2} R_{y y}}{\partial t^{2}}=-\frac{2}{\bar{\rho}^{2}} \frac{\partial \bar{p}}{\partial y} \frac{\partial \bar{\rho}}{\partial y} R_{y y}+\frac{2 b}{\bar{\rho}}\left(\frac{\partial \bar{p}}{\partial y}\right)^{2}
$$

The second term on the right, in the qualitative fashion of the other terms here omitted, is not relevant to this discussion, while the first term on the right contributes either an oscillatory component (if $\left(\frac{\partial \bar{p}}{\partial y}\right)\left(\frac{\partial \bar{p}}{\partial y}\right)>0$ ) or an exponentially growing component (if $\left(\frac{\partial \bar{p}}{\partial y}\right)\left(\frac{\partial \bar{p}}{\partial y}\right)<0$ ). This last would appear to be the classic linear Rayleigh-Taylor solution but fails in that regard in one crucial respect.

To demonstrate this failure and its remediation, we note that, as it is written in local form, the first term distributes the turbulence energy only within the mixing layers itself. In that region for the unstable case, $\mathbf{R}_{\mathrm{yy}}$ grows as 


$$
R_{y y}(t)=R_{y y}(0) \exp \left[t \sqrt{\frac{2}{\bar{\rho}^{2}}\left|\frac{\partial \bar{p}}{\partial y} \frac{\partial \bar{\rho}}{\partial y}\right|}\right] .
$$

Letting $g \equiv(1 / \bar{\rho})(\partial \bar{p} / \partial y)$ and $\partial \bar{\rho} / \partial y=\Delta \rho / w$, where $\Delta \rho$ is the overall density difference across the mixing layer of width $\mathrm{W}$, we get

$$
R_{y y}(t)=R_{y y}(0) \exp \left[t \sqrt{\frac{2}{W}\left|g \frac{\Delta \rho}{\bar{\rho}}\right|}\right] .
$$

In contrast, for an infinitesimal velocity squared, classic linear Rayleigh-Taylor analysis gives (Harlow and Amsden, 1971)

$$
R_{y y}(t)=R_{y y}(0) \exp \left[2 t \sqrt{k\left|g \frac{\Delta \rho}{2 \bar{\rho}}\right|}\right] .
$$

(Note the factor of 2, to describe growth of velocity-squared.) The essential difference between these two results is the occurrence of $1 / \mathrm{W}$ in the first and $k$ in the second. In both cases, $R_{y y}(0)$ may depend on $k$, but the turbulence-transport result is essentially independent of $k$ in the exponent. The reason, of course, is that the creation of $R_{y y}$ in the purely local formulation confines the inertial resistance to growth to the mass within the mixing layer itself. In reality, as described above, the mass that must be set in motion extends well beyond the edges, so that $\mathrm{R}_{\mathrm{yy}}$ can be expected to grow with a much reduced exponent for structures that are large compared to $\mathrm{W}$, i.e., for $\mathrm{kW} \ll 1$. Thus this local formulation preserves the spectral structure of $R_{\mathrm{yy}}(0)$.

With nonlocality of $Q\left(y^{\prime}, y\right)$, the turbulence-transport results account for the added inertia and give the classical exponential growth. As a practical consequence, the behavior of TMZ growth and appearance is significantly altered; as a conceptual consequence, the influence of low wave numbers on self-similarity is quite different from that of the purely local theory. The nonlocal formulation does not preserve the spectral structure of $R_{y y}(0)$.

To demonstrate the remedial effects of including the nonlocal pressure-wave propagation, we return to the equation for $R_{y y}$, Eq. (V.5), which is rewritten as follows:

$$
\frac{\partial R_{y y}}{\partial t}=\int_{-\infty}^{+\infty} S\left(y^{\prime}\right) k \exp \left[-2 k\left|y^{\prime}-y\right|\right] d y^{\prime},
$$

where 


$$
S(y) \equiv 2 a \frac{\partial \bar{p}}{\partial y}
$$

To isolate the essence of our demonstration, it is sufficient to consider, for $y>W / 2$, the approximation (an over estimation by choosing $S(y)$ at $y=0$ )

$$
\begin{aligned}
\frac{\partial R_{y y}}{\partial t} & =\int_{-W / 2}^{W / 2} S(0) k \exp \left[-2 k\left|y^{\prime}-y\right|\right] d y^{\prime} \\
& =S(0) \exp [-2 k y]\left(\frac{\exp (k W)-\exp (-k W)}{2}\right) .
\end{aligned}
$$

Twice the integral from W/2 to $\infty$ thus gives the total amount of $\mathbf{R}_{\mathrm{yy}}$ created per unit time outside of the TMZ, which accordingly is

$$
\frac{\partial}{\partial t} R_{y y}(\text { outside })=S(0)\left[\frac{1-\exp (-2 k W)}{k}\right] .
$$

The total amount created per unit time everywhere is (because of the normalization of $Q$ ) the same as the local-theory total amount, namely $\mathrm{S}(0) \mathrm{W}$. Thus the nonlocal prediction for total $\mathrm{R}_{\mathrm{yy}}$ created inside the layer per unit time is

$$
\frac{\partial}{\partial t} R_{y y}(\text { inside })=S(0) W\left[1-\frac{1-\exp (-2 k W)}{2 k W}\right] .
$$

For $\mathrm{kW}<1$, we expand the exponent to get

$$
\frac{\partial}{\partial t} R_{y y}(\text { inside }) \approx S(0) k W^{2} .
$$

Divide this by $\mathrm{W}$ to get, within the layer,

$$
\frac{\partial R_{y y}}{\partial t}=2 a k W \frac{\partial \bar{p}}{\partial y} .
$$

With this alteration the exponential growth for $\mathrm{kW}$ small becomes

$$
R_{y y}(t)=R_{y y}(0) \exp \left[t \sqrt{2 k\left|g \frac{\Delta \rho}{\bar{\rho}}\right|}\right],
$$


which now contains the essential dependence on $\mathrm{k}$ seen in the solution to the classic linear Rayleigh-Taylor analysis, Eq. (V.10).

It is interesting to contrast our approach to nonlocal pressure-wave effects with the technique employed by Demuren et al. (1994). They describe a procedure for "local diffusion sources to be distributed over lengths of the order of the integral scale." They implement this technique into a nonspectral transport model and report that it "enabled the well-known free-stream edge singularity problem to be eliminated." To the extent that turbulence self-diffusion receives contributions from the nonlocal triple-correlation terms that arise from pressure-velocity correlations through Greensfunction integral solutions, we agree that turbulence diffusion should be distributed nonlocally. This effect is physically visualized in a two-point (spectral) formulation of the theory. To capture the effects of inertial response on the creation of turbulence (and thus the Rayleigh-Taylor linear growth rate), we believe the principal nonlocal effect must be as described by our $Q\left(y^{\prime}, y\right)$ modification to the differential-acceleration term (and to the mean-flow shear coupling).

It is of interest to note the relation between linear Rayleigh-Taylor analysis and the self-similar analysis for the turbulence equations described in Section VII. If there is a rich spectrum of modes at $t=0$, then each will grow exponentially, then saturate, creating an envelope for net growth of the fully nonlinear evolution (see Fig. V.1).

This envelope has the quadratic behavior dictated by the dimensionality of acceleration, $g$. A heuristic analysis of this process starts with the linear growth equation for amplitude, $\mathrm{A}$, at wave number $\mathrm{k}$, and Atwood number At

$$
\frac{\mathrm{d}^{2} \mathrm{~A}}{\mathrm{dt}^{2}}=\mathrm{kg}(\mathrm{At}) \mathrm{A}
$$

and replaces $\mathrm{k}$ by $1 / \mathrm{S}$, where $S$ is the currently dominant scale at which growth is occurring. With self-similarity, $S$ is a fixed multiple of $A$; that is, $S=\beta A$. Then

and

$$
\frac{\mathrm{d}^{2} \mathrm{~A}}{\mathrm{dt}^{2}}=\frac{\mathrm{g}(\mathrm{At})}{\beta},
$$

$$
A=\frac{1}{2 \beta}(A t) g t^{2} .
$$

If mode saturation were to occur when the magnitude of $A$ is approximately $20 \%$ of $S$, then we get $\mathrm{A}=0.1 * \mathrm{At} * \mathrm{~g} * \mathrm{t}^{2}$, which is consistent with the actually observed self-similar behavior of a TMZ, for which experiments give a coefficient of about 0.12 . 


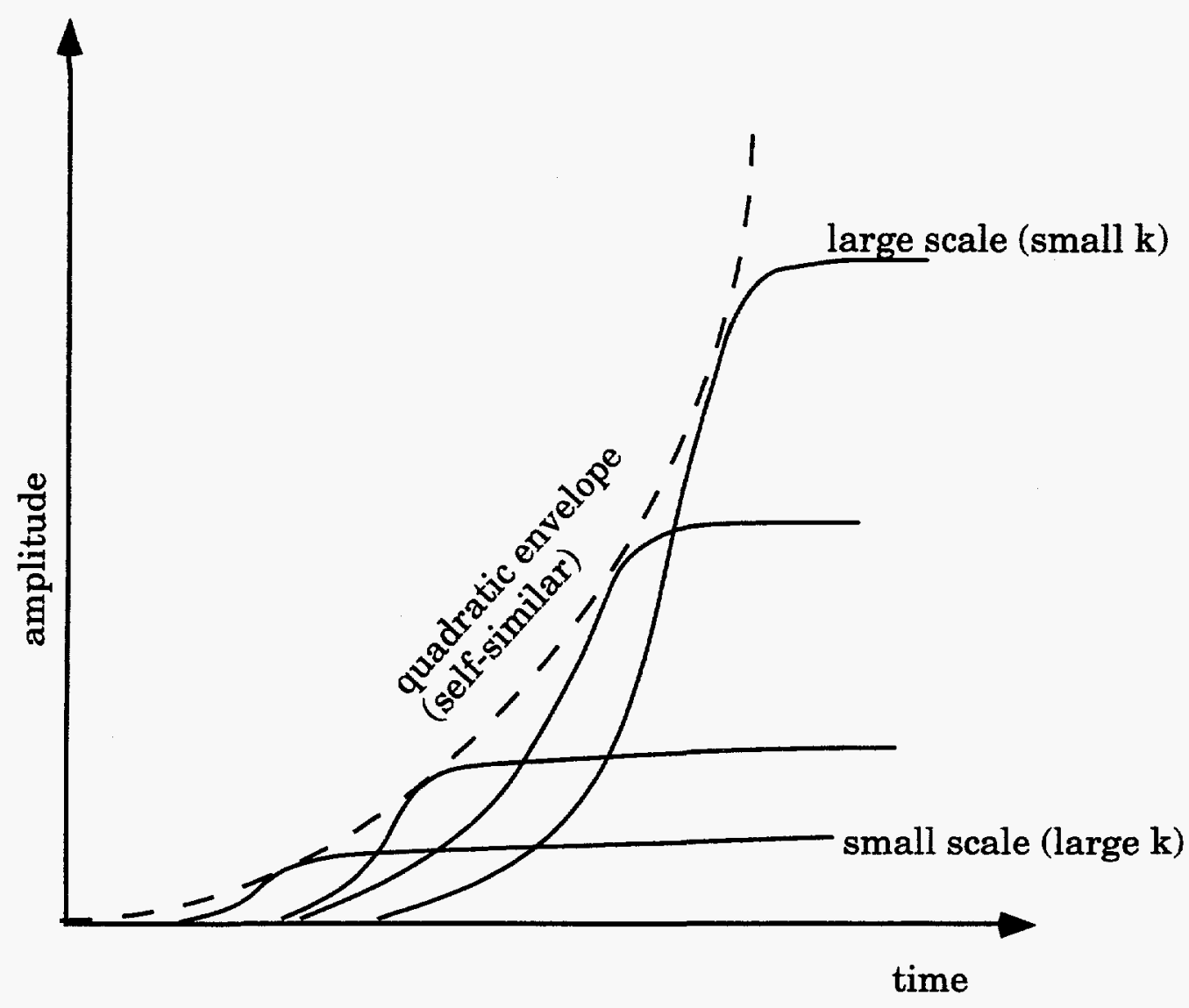

Fig. V.1. Schematic to demonstrate the growth and saturation of initial perturbations of the Rayleigh-Taylor instability. 


\section{SELF-SIMILAR FORM FUNCTIONS}

The concept of statistical self-similarity is demonstrated in Section VII for the example of a turbulent mixing zone between two immiscible fluids. As an idealization, the zone extends laterally in all directions and all quantities are statistically independent of the lateral dimensions. Indeed we will assume that ensemble averaging can be replaced by lateral spatial averaging, with results that vary in time only as functions of wave number and the distance normal to the layer. Such an idealization does not exist in nature, but there are nevertheless numerous circumstances in which it serves as a good approximation.

Besnard et al. (1990) showed that statistical self-similarity is a crucial element for the validity of single-point turbulence transport models. In order to describe with a small number of variables the collective effects of a virtually infinite number of degrees of freedom, it is clear that severe constraints must exist to confine the dynamics of the fluid to a very small set of all possibilities. Moreover, one of the necessities for single-point applicability is that the return to self-similarity occur rapidly after a change in the external drive conditions.

Self-similarity of the statistics is characterized by statistical form functions. They always occur with the same structure for each set of mean flow circumstances after proper space and time scalings are performed. Universal form functions, however, are idealizations that never precisely occur in nature, although in many situations of interest they are closely approximated. The forms of such functions may strongly reflect the existence of anisotropy and may even depend on persisting conditions in the limit as $|\mathbf{k}| \rightarrow 0$. Our ultimate goal is to identify these form functions in six-dimensional $\mathbf{k}, \mathbf{x}$ space. Subsets can be found in $\mathbf{k}$ or $\mathbf{y}$ space alone and in various other combinations of the wave number and position-vector components. Complete analytical derivation is difficult (see Besnard et al. (1990)); partial derivations are well known for simple cases (e.g., the $\mathrm{k}^{-5 / 3}$ inertial range for $R_{\alpha \alpha}$; more general extraction may only be possible numerically; see Section VII).

The simplest statistical self-similar forms occur in the decay of homogeneous isotropic decaying turbulence in a constant density fluid with vanishing viscosity. Besnard et al. (1990) discuss this form and use an anisotropic generalization to derive spectral moments. They show that with this simple idealization, the single-point equations (e.g., K- $\varepsilon$ models) can be derived. These types of single-point equations have been successfully applied to circumstances far beyond the case of homogeneous anisotropic decay.

In general, self-similarity for the behavior of some turbulence statistic like $R_{i j}(y, k, t)$, means that a scaling law can be found for its variations in magnitude, such that the scaled quantity is then a function only of the combined variables, $y / L(t)$ and $k L(t)$, in which $L(t)$ is a function with the 
dimensions of length. Thus the function of three variables, $\mathrm{y}, \mathrm{k}$, and $\mathrm{t}$, has been transformed to a function of two variables.

This scaling of magnitudes and the independent variables that define self-similarity can be described more precisely. Consider the behavior of

$$
E(y, k, t)=\frac{1}{2 \bar{\rho}} R_{n n}(y, k, t),
$$

which has the dimensions of (distance) $\left.)^{3 /(t i m e)}\right)^{2}$. Suppose we scale the space and time coordinates such that

$$
\begin{aligned}
& y \rightarrow \omega^{n} y \\
& k \rightarrow \omega^{-n} k \\
& t \rightarrow \omega^{m^{m}},
\end{aligned}
$$

where $\omega$ is a dimensionless number that defines the scale, while $m$ and $n$ are numbers to be specified. We also scale the magnitude of $\mathrm{E}$ according to its dimensionality,

$$
E \rightarrow \omega^{3 n-2 m} E .
$$

Our constraint of self-similarity in this process results in the equation

$$
\omega^{3 n-2 m} E(k, y, t)=E\left(\omega^{n} y, \omega^{-n} k, \omega^{m} t\right) .
$$

Differentiate with respect to $\omega$ and then set $\omega=1$. The result is

$$
(3 n-2 m) E=n y \frac{\partial E}{\partial y}-n k \frac{\partial E}{\partial k}+m t \frac{\partial E}{\partial t},
$$

which can be solved to give

$$
E(y, k, t)=t^{\frac{3 n-2 m}{m}} \hat{F}\left[\frac{y}{t^{n / m}}, k t^{n / m}\right],
$$

in which $\hat{\mathrm{F}}$ is an arbitrary function of its arguments. The origin of time is arbitrary, so that we may choose the onset of self-similarity at some time, $\mathrm{t}_{0}$, and write 


$$
\begin{gathered}
\mathrm{L}(\mathrm{t}) \equiv \mathrm{L}_{0}\left(t-t_{0}\right)^{\mathrm{n} / m} \\
\mathrm{~K}(\mathrm{t}) \mathrm{L}(\mathrm{t}) \equiv \mathrm{K}_{0} \mathrm{~L}_{0}\left(t-t_{0}\right)^{\frac{3 n-2 m}{m}}
\end{gathered}
$$

thus re-expressing the solution in the form

$$
E(y, k, t)=K(t) L(t) F\left[\frac{y}{L(t)}, k L(t)\right]
$$

with $\mathrm{F}$ having absorbed some constants and likewise being an arbitrary function of its arguments. Note that in this form

$$
\int_{0}^{\infty} E(y, k, t) d k=K(t) G\left(\frac{y}{L(t)}\right),
$$

where

$$
G(\eta) \equiv \int_{0}^{\infty} F(\eta, \xi) d \eta
$$

This form for $E(y, k, t)$ describes a constraint of self-similarity It is derived without reference to the transport equation for $\mathrm{E}$ and serves to distinguish from all possible solutions a particular subset with a type of self-similarity.

Dimensional arguments for the spectrally integrated quantities are based on the TMZ growth having "forgotten" all finite scale details of the initial conditions so that $\mathrm{g}$ is the only dimensional scaling parameter. Thus any velocity scale must vary as gt while the turbulence energy per unit mass varies as $(\mathrm{gt})^{2}$ and length scales vary as $\mathrm{gt}^{2}$. Thus $\mathrm{n}$ and $\mathrm{m}$ are constrained to $\mathrm{n}=2 \mathrm{~m}$.

Applied to homogeneous, isotropic turbulence (independent of $y$ ), the result of self-similarity is conversion of a partial differential equation for $E(k, t)$ to an ordinary differential equation for $\mathrm{F}[\mathrm{kL}(\mathrm{t})]$. Besnard et al. (1990) discuss in detail the properties of $\mathrm{F}$.

For the mixing-layer analysis in $y-k$ space, the self-similarity constraint has likewise reduced the number of independent variables; but insertion into the transport equation nevertheless results in a partial differential equation for $F(y / L, k L)$, which has a much greater richness of possible solutions than the ordinary differential equation for homogeneous, isotropic circumstances. We thus see hints of possible nonuniqueness of self-similar mixing-layer turbulence. 
The breadth of possibilities increases substantially when we examine the behavior in sixdimensional circumstances. Recognizing the dimensionality of $R_{i j}(x, k, t) / 2 \rho$ as

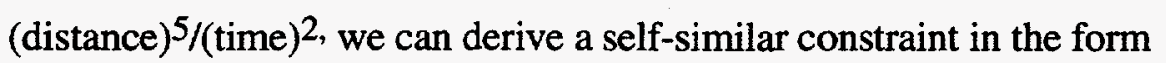

$$
\frac{R_{i j}(x, k, t)}{2 \rho} \equiv K(t) L^{3}(t) F_{i j}\left[\frac{x}{L(t)}, k L(t)\right],
$$

in which

$$
\begin{aligned}
L(t) & \equiv L_{0}\left(t-t_{0}\right)^{n / m} \\
K(t) L^{3}(t) & \equiv K_{0} L^{3} 0\left(t-t_{0}\right)^{\frac{5 n-2 m}{m}} .
\end{aligned}
$$

Insertion of this form into the full transport equation reduces the seven independent variables in the partial differential equation to six independent variables. For the mixing layer with physical-space dependence on y, only, the number of independent variables is four. We are in the process of examining some of the properties and consequences of this more general self-similarity for the turbulence of a single fluid with constant density, but such discussions for variable-density fluids are beyond the scope of this present study.

We are, however, concerned with extensions to the self-similar form in our special case of the mixing layer. We rewrite Eq. (VI.8) with a different combination of variables in the F function (which is perfectly allowable); i.e.,

$$
E(y, k, t)=K(t) L(t) F\left[\frac{y}{L(t)}, k L(t) G\left(\frac{y}{L(t)}\right)\right] .
$$

We hypothesize that the $G$ function can be found in such a way that

$$
F\left[\frac{y}{L(t)}, k L(t) G\left(\frac{y}{L(t)}\right)\right]=F_{1}\left(\frac{y}{L(t)}\right) F_{2}\left[k L(t) G\left(\frac{y}{L(t)}\right)\right] .
$$

This hypothesis is capable of being tested numerically. It states in effect that the spectrum is self-similar in k-space with a shift in maximum and position thereof for which $\mathrm{kL}(\mathrm{t})$ depends only on $\eta$, where $\eta=y / L(t)$. The first realization of this form was described by Besnard et al. (1990) on the basis of numerical solutions of their spectral model equations for a constant-density fluid in a temporally evolving shear layer. Section VII of this report describes a further realization for the turbulent mixing of two fluids. 
We are led to define a length scale $\mathrm{L}(\mathrm{y}, \mathrm{t})$ that varies with both position and time, as was assumed by Besnard et al. (1990) for the spectral-moment derivations of single-point transport equations. Thus we define $L(y, t)$ as being equal to $L(t) G(\eta)$, and $K(y, t) L(y, t)$ as identically being equal to $F_{1}(\eta) L(t) K(t)$. Then Eqs. (VI.13) and (VI.14) can be written as

$$
E(y, k, t)=K(y, t) L(y, t) F_{2}[k L(y, t)] .
$$

Even more generally, if the mixing layer is of finite lateral extent or curved away from planarity, we postulate the concept of localized self-similarity with $K(x, t)$ and $L(x, t)$, which is the actual form used for deriving moment equations for turbulence in a constant density fluid. In this more general case, we believe that the decomposition described in Eqs. (VI.13) and (VI.14) is also relevant. 


\section{NUMERICAL RESULTS FOR THE SPECTRAL TURBULENCE TRANSPORT EQUATIONS}

\section{A. Introduction}

In this section, we present the numerical results of the spectral equations from Section IV as applied to turbulent mixing by the Rayleigh-Taylor instability. The purpose of this section is to demonstrate the behavior of a base case of the spectral equations of Section IV, show comparisons with experiments, and show the effect of some variations to the base case. For this demonstration, we will present numerical results of a base case for mixing only, followed by examples to demonstrate the influence that the various coefficients have on the calculations. We also show the spectral behavior for the net mass flux velocity, a, during the process of a rapid transient (a gravity reversal is used for this demonstration).

To study the properties of the spectral equations we have written a finite-difference code for their numerical solution. The configuration we have chosen for illustration is that of an initial interface between two nonviscous, incompressible fluids of different densities, subjected to a pressure gradient normal to the interface. The pressure gradient arises by imposing a body force ("gravity") to the fluids but holding the containing vessel at rest. An equivalent procedure, employed by the experiments with which we compare, accelerates the vessel, with a pressure gradient arising in order that the fluid is accelerated commensurate with the motion of the vessel.

The numerical solution technique and its validation for the present purposes is described by Steinkamp (1995). The computer code consists of a spectral part and a spectrally-integrated part. In the latter, with the containing vessel at rest, the pressure gradient is updated in such a manner as to ensure vanishing flux of material volume at all stations within the flow. Thus the transport equations for $\tilde{\mathbf{u}}$ and the spectrally integrated transport equation for a are forced to be equivalent, which determines the variations of pressure in response to the specified body force.

The principal data for comparison with calculations comes from extensive experimentation at AWE (Smeeton and Youngs, 1987) and Chelyabinsk-70 (Kucherenko et al., 1991). The data are not as complete as we would like; we expect considerable more data from the anticipated experiments of Remington and Dimonte at the LLNL (personal communication, 1995). The

results currently available are profiles of estimated mix fraction across the layer, the mix interpenetration rates into each fluid, and some qualitative indications of dominant scale.

\section{B. Mixing Due to an Acceleration}

Figures VII.B.1-VII.B.12 give results from a base-case calculation. In the following figures, the spectral behavior of the variables is plotted as functions of the nondimensional variable $z$, where $\mathrm{z}=\mathrm{Ln}\left(\mathrm{k} / \mathrm{k}_{0}\right)$. This logarithmic transformation is used to accentuate the regions of physical significance; that is to say, as the turbulence evolves the turbulent length scales grow, 
corresponding to a migration of the turbulence spectra toward $\mathrm{k}=0$. Thus the transformation allows the details of the spectrum near $\mathrm{k}=0$ to be spread over the corresponding infinite interval in nondimensional $\mathrm{z}$-space. The constant $\mathrm{k}_{0}$ serves to nondimensionalize the transformed spectral equations and to provide a reference wave number for specifying initial conditions. The initial configuration consists of a heavy fluid $(\rho=2.0)$ sitting over a lighter fluid $(\rho=1.0)$. At the time $t=0$, acceleration $(g=-1.0)$ is turned on and the mixing of the two fluids commences. For this calculation, the values of the coefficients are $C_{1}=0.1212, C_{2}=0.0606, C_{f b}=0.5$, $C_{D}=0.03, C_{r p 1}=5.0, C_{r p 2}=6.0, C_{m}=1.0$, and $C_{b d}=0.0$. The nonlocal version of the source term is used in the Reynolds-stress equations. At $t=0$, the values of $R_{n n}(k), R_{y y}(k)$, and $a(k)$ are zero in every computational cell. The value of $b(k)$ likewise vanishes in every cell except the one containing the two-fluid interface, where

$$
b(k)=\frac{\gamma_{1} k^{n}}{1+\gamma_{2} k^{n+5 / 3}} .
$$

The two coefficients, $\gamma_{1}$ and $\gamma_{2}$, have been chosen to ensure that the maximum of $b(k)$ occurs at $k=k_{0}$ and the spectral integral of $b(k, y)$, namely $b(y)$, has the maximum configurational value corresponding to equal volumes fractions, $\alpha$, and $\alpha_{2}$, of the two fluids in that cell; i.e., $\alpha_{1}=\alpha_{2}=0.5$,

$$
\mathrm{b}=\frac{\left(\rho_{2}-\rho_{1}\right)^{2}}{4 \rho_{1} \rho_{2}}
$$

For a discussion on the configurational value of $b$, see Besnard et al. (1992). For the base case, $\mathrm{n}=4.0, \mathrm{k}_{0}=1.0$, and

$$
\begin{aligned}
& \gamma_{1}=\frac{\left(\rho_{1}-\rho_{2}\right)^{2}}{4 \rho_{1} \rho_{2} f(n)}, \\
& \gamma_{2}=\left(\frac{3 n}{5}\right) k_{0}^{-(n+5 / 3)},
\end{aligned}
$$

where,

$$
f(n)=\int_{-\infty}^{+\infty} \frac{k^{n} d k}{1+(3 n / 5)\left(k / k_{0}\right)^{n+5 / 3}}
$$


Boundary conditions in y space are of no significance because each calculation is terminated before the influence due to the nonlocal pressure effects has reached the edges of the computational mesh. Boundary conditions in $\mathrm{k}$ space attach the low and high wave-number behavior for each variable to a power-law variation of $k$. For $b(k)$ we have also used a boundary condition at high wave numbers that blocks the cascade flux, but for the base case the flux is not blocked.

Figure VII.B.1 illustrates the time evolution of the spectral behavior for the variables as functions of the logarithm of $\left(\mathrm{k} / \mathrm{k}_{0}\right)$. In all four plots the spectra are migrating to the left (toward lower $\mathrm{k}$ values) as time progresses. We identify length scales as the reciprocal of the wave number at which the spectrum has its extremum. The leftward migration is thus consistent with an increasing length scale for each variable. The spectra are given for equal time increments,

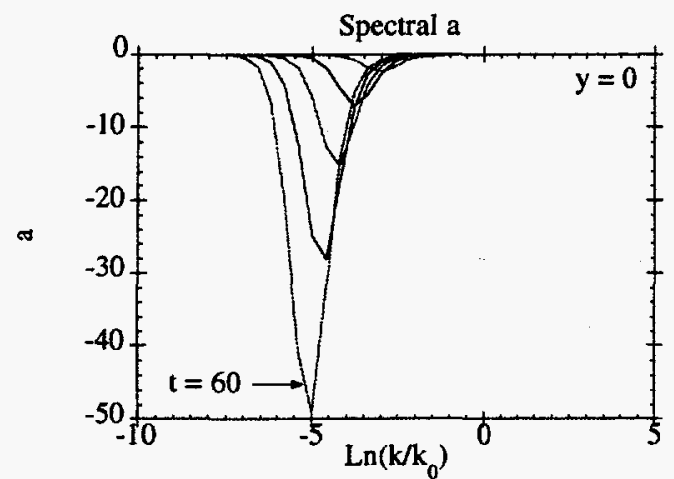

(a)

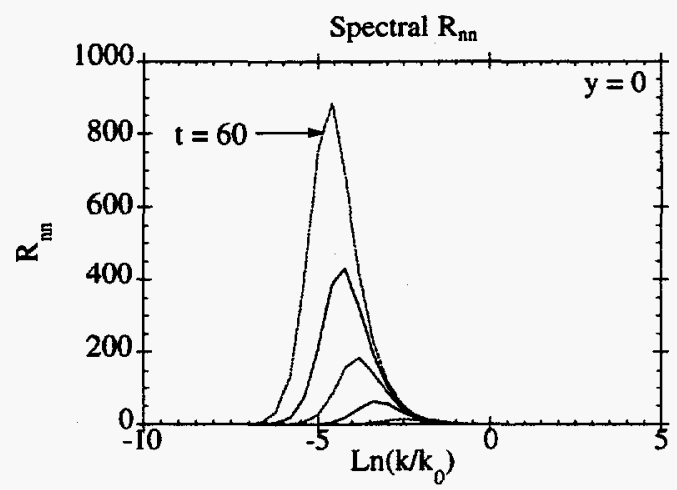

(c)

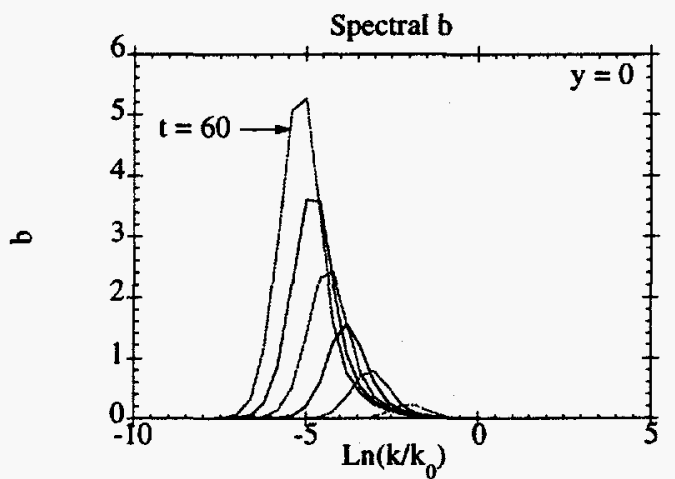

(b)

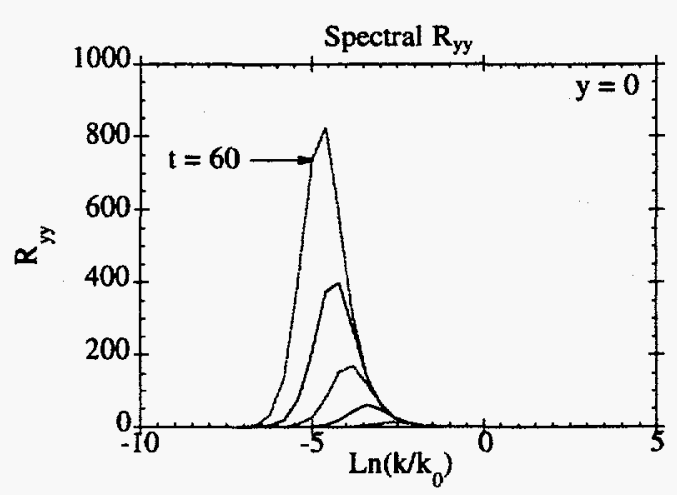

(d)

Fig. VII.B.1. Spectra at times $t=10,20,30,40,50$, and 60 , at the position of the initial fluid interface: (a) the net mass-flux velocity, $a,(b)$ the specific volume-density correlation, $b,(c)$ the contraction of the Reynolds stress tensor, $R_{n n}$, and $(d)$ the $R_{y y}$ component of the Reynolds stress tensor. 
$\Delta t=10$, so as to demonstrate the linearly increasing growth rate of the peaks of the spectra. This variation is consistent with quadratic growth in time of the TMZ width, in agreement with expected self-similar behavior as described in Section VI.

One characteristic that demonstrates the intervariable relationships that exist within the equations is the differing behavior of the spectral structures at the higher wave numbers. The right sides of the spectra for $a$ and $b$ migrate rapidly toward smaller values of $k$ causing an overlapping appearance for a sequence of plots, while the right sides of the spectra for $R_{i j}$ coincide for that same sequence of times. The strong migration to the left seen in the $b$ spectrum is due to the relatively large value chosen for $\mathrm{C}_{\mathrm{fb}}$, the one free parameter in our equations. The value of $\mathrm{C}_{\mathrm{fb}}$ significantly influences the leftward migration of the $\mathrm{b}$ spectrum. This effect influences the $b$ spectrum the most. Because $b$ is a principal source to $a$, the effect is also seen in the a spectrum but to a relatively lesser degree. The Reynolds-stress spectra are, in turn, also moved to lower wave numbers. The effects are associated with nonlinear bubble-doubling in the mixing layer, as observed in numerous experiments and direct-numerical-simulation calculations, and are important for the agreement with experiments described below. These overall spectral forms result from a complex interplay among physical processes represented by the terms in the equations. In Section VIII we show in much more detail the origins for the behaviors described in this base-case calculation and its variants.

The self-similar nature of the spectral evolution of Fig. VII.B.1 can be demonstrated by plotting the results in the form of Fig. VII.B.2. The plots are normalized in space so as to enable us to compare the shapes of these plots at successive times. The resulting normalized plots are called shape plots. To achieve this normalization two steps are taken. First, a spectrum is divided by its extremum, resulting in all of the spectral maxima coinciding with 1.0 and values of the entire spectrum falling between 0 and 1 . Next, the spectra are all shifted such that the maximum of the spectra coincide with $\mathrm{k}=\mathrm{k}_{0}$. A sequence of times of these resulting shape plots are given on the same graph so as to identify any differences in their structures. The coincidence of all plots indicates a self-similar evolution of the mixing layer. The plots of Fig. VII.B.2 are taken from the cell where the initial fluid interface existed. The mixing evolution becomes selfsimilar near $t=30$, and this is demonstrated by the overlapping plots of this figure.

Notice the consequence of shifting the spectra so that their peaks coincide with $k=k_{0}$. As the spectral peak migrates to the left, less and less of the spectrum lies to the left of that peak and more of the resolved spectrum lies to the right. When the spectra are then shifted in the normalizing process, the appearance of a diminishing tail on the left side and an increasing tail on the right side is observed. Thus the plot with the longest tail on the left side represents the earliest time of the sequence and the plot with the longest tail on the right side represents the latest time in the sequence. The slopes at low and high wave numbers are given to demonstrate 
the behaviors of the spectral variables. The interpretation and significance of these forms and slopes is discussed in Section VIII. It is especially noteworthy to observe here the very close achievement of self-similarity.

The slopes in Fig. VII.B.2 represent the power law behavior of the spectra at only the initial position of the fluid interface, $y=0$. Since these plots are functions of the logarithm of $\left(\mathrm{k} / \mathrm{k}_{0}\right)$, the slope of the line corresponds to the power, $n$, of the wave number, $k$. (These are slopes near $\mathrm{k}=0$ and represent modifying tendencies for low wave numbers near $\mathrm{k}=0$, but not at precisely $k=0$, where the initial value of $n$ is preserved.) The spatial variation of the value of $n$ across the TMZ for both the low and high wave numbers is shown in Fig. VII.B.3. This plot is given for $t=65$ well after the establishment of self-similarity. In effect, the spatial variations shown in Fig. VII.B.3 indicate the temporal evolution of the power laws for any one fixed position. The

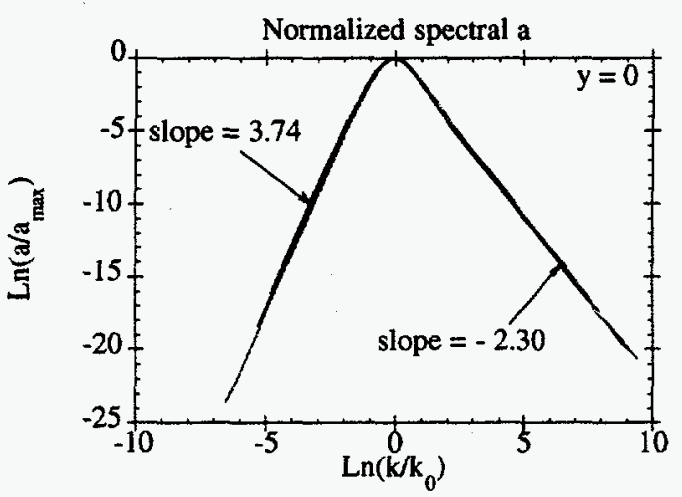

(a)

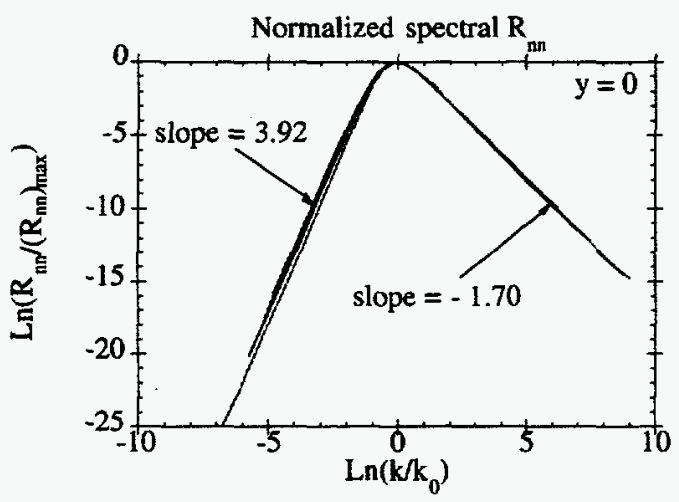

(c)

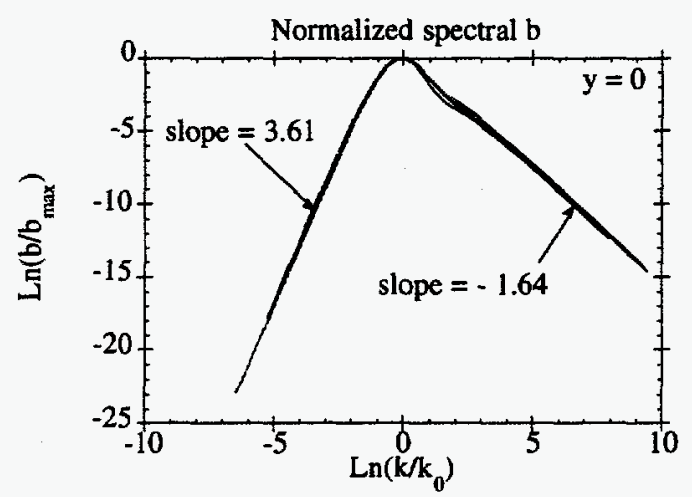

(b)

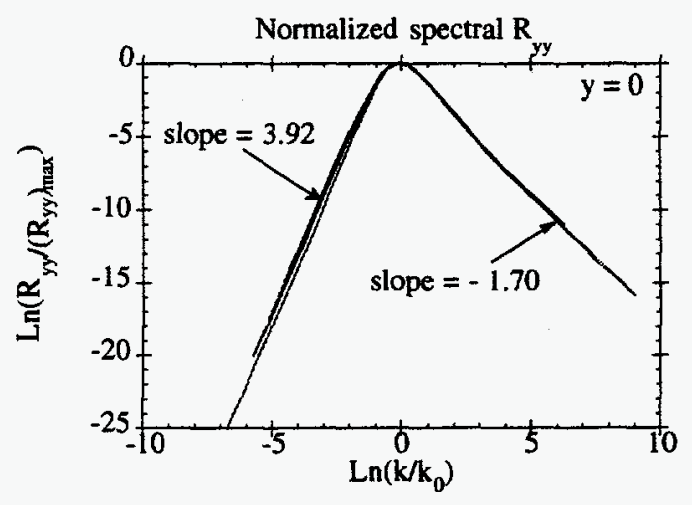

(d)

Fig. VII.B.2. Normalized spectra for $t=30,35,40,45,50,55,60$, and 65 , at the position of the initial fluid interface: (a) the net mass-flux velocity, a, (b) the specific volume-density correlation, $b$, (c) the contraction of the Reynolds stress tensor, $R_{n n}$, and (d) the $R_{y y}$ component of the Reynolds stress tensor. 


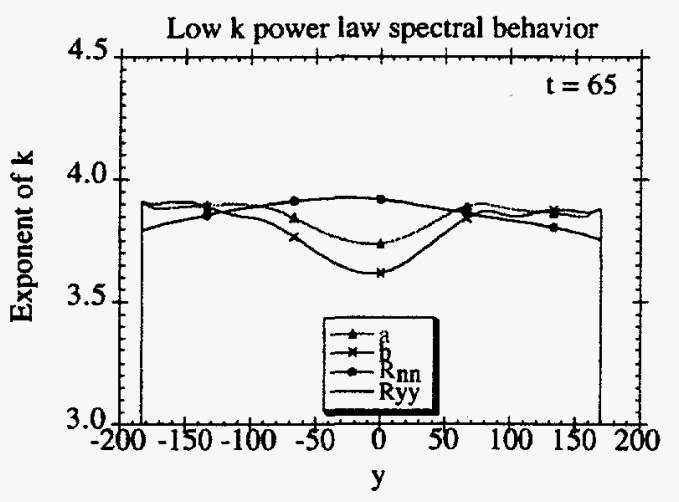

(a)

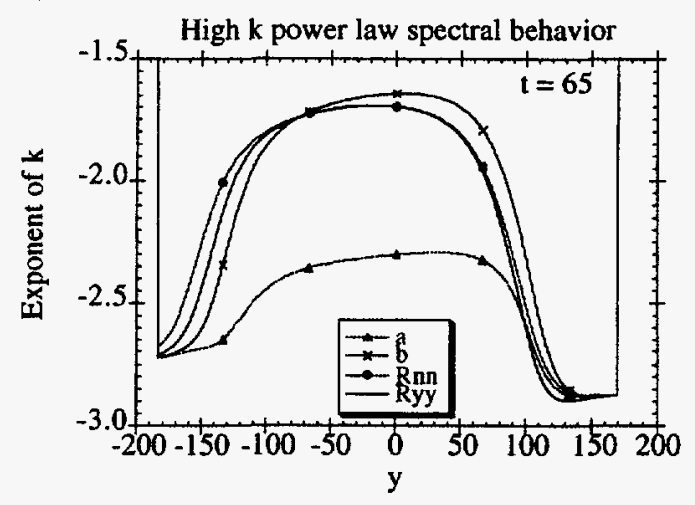

(b)

Fig. VII.B.3. Profiles of the power law exponent across the TMZ at $t=65$ for (a) the low wave numbers, and $(b)$ the high wave numbers.

center of the plot $(y=0)$ has been developing the longest, and the edges of the TMZ are, relatively speaking, the newest parts. For the low wave numbers, the $b(k)$ spectrum has a smaller value of $n$ near $y=0$ than occurs for the other spectra. The explanation lies in the effects of the $\mathrm{C}_{\mathrm{fb}}$ term, which gives to $\mathrm{b}(\mathrm{k})$ the leading role in describing the nonlinear bubble-double process. Initialized with $\mathrm{k}^{4}$ variation near $\mathrm{k}=0$, the $\mathrm{b}(\mathrm{k})$ spectrum feels most strongly the modifying effects of leftward (in $\mathrm{k}$ space) propagation of a concave-downwards structure that decreases the exponent. With $b(k)$ as a source to $a(k)$, and $a(k)$ as a source to $R_{i j}(k)$, the decrement in exponent is successively less in these latter two functions. This behavior, and likewise, the behavior of both the $a(k)$ and the $R_{i j}(k)$ spectra is consistent with the overlapping seen in Fig. VII.B.1. The high wave number power law behavior of the four variables coincides at the edges of the TMZ and then separates as time evolves. The values of $\mathrm{n}$ for the high wave numbers of Fig. VII.B.3(b) at $y=0$ are equal to the slopes of the plots in Fig. VII.B.2. Only near $y=0$ does one observe the classical values of $n=-5 / 3$ [for $b(k)$ and $\left.R_{i j}(k)\right]$ and $n=-7 / 3$ for $a(k)$, which result from the constancy of cascade flux for $b(k), R_{n n}(k)$, and $R_{y y}(k)$ and the balance of drag with production for $\mathrm{a}(\mathrm{k})$, as discussed in more detail in Section VIII.

Figure VII.B.4 shows the evolving spectral forms at a distance of 50 units of length below the initial interface, showing the manner of arrival of the spectrum at each station as the TMZ widens. The spectrum of $\mathrm{a}(\mathrm{k})$ arrives at nearly its self-similar form for all wave numbers, whereas the spectra for the other quantities arrives in self-similar forms only for the lower wave numbers; at the higher wave numbers there is significant lag in the establishment of spectral selfsimilarity. There are two reasons for this behavior. One of these is the effect of nonlocal source 


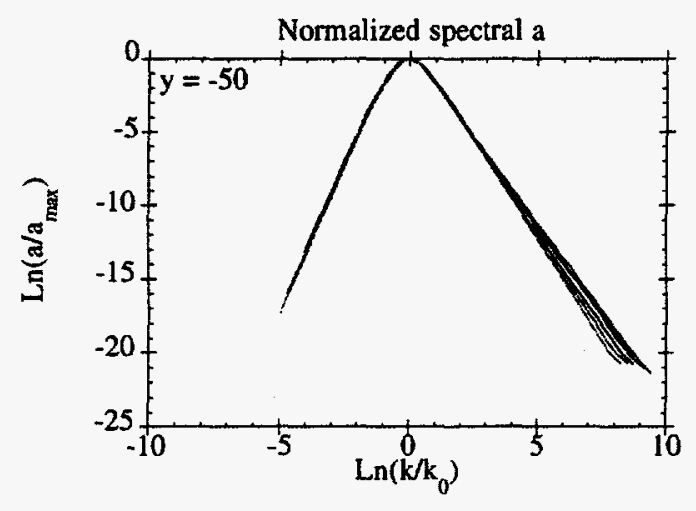

(a)

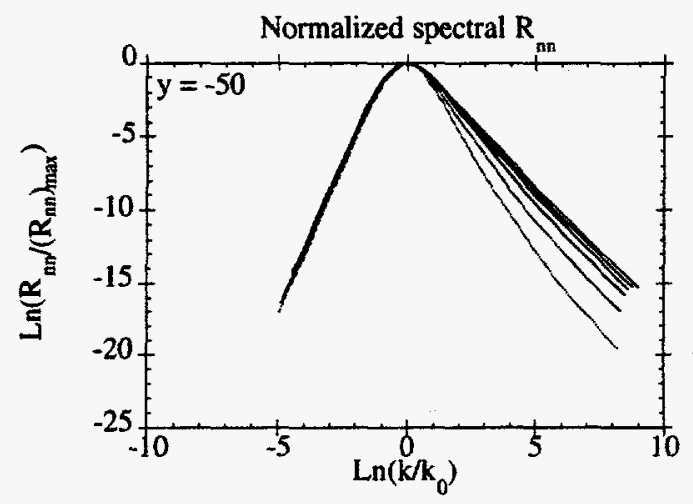

(c)

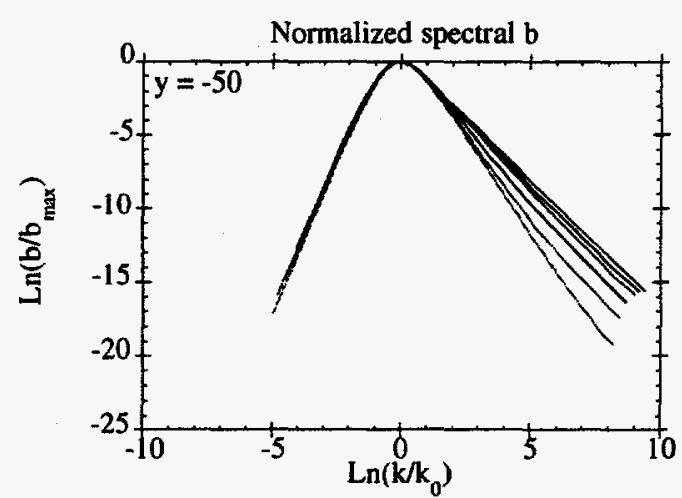

(b)

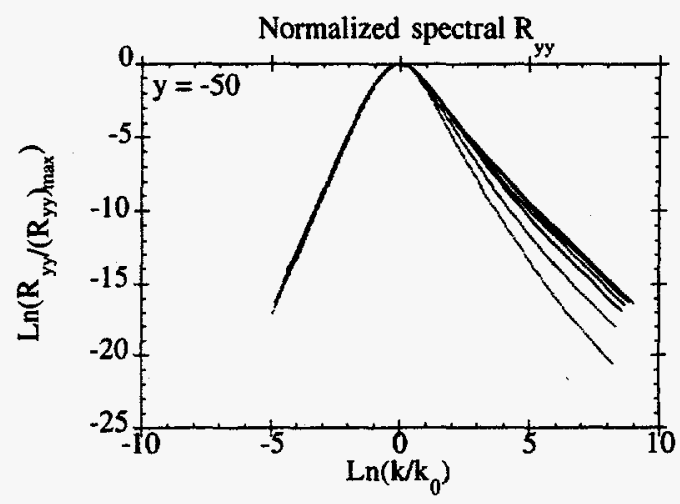

(d)

Fig. VII.B.4. Normalized spectra for $t=30,35,40,45,50,55,60$, and 65 , a distance of 50 units of length below the centerline of the TMZ: (a) the net mass-flux velocity, (b) the specific volume-density correlation, (c) the contraction of the Reynolds stress tensor, and $(d)$ the $R_{y y}$ component of the Reynolds stress tensor.

for $R_{n n}(k)$ and $R_{y y}(k)$, which projects the low-wave number effects into lateral regions well ahead of the effects at high wave numbers. The second reason is that the growth of spectral forms for $b(k), R_{n n}(k)$, and $R_{y y}(k)$ is at first dominated by the effects of sources which at any station tend initially to impart a $\mathrm{k}^{-7 / 3}$ form at high wave numbers, being thereafter altered to $k^{-5 / 3}$ as the effects of constant cascade flux begin to dominate.

Figure VII.B.5 shows a comparison at $\mathrm{t}=65$ of the spectral form functions at $\mathrm{y}=0$ and $y=-50$. By this time the self-similar form has been completely reached at $y=0$ and nearly so at 50 length units below $y=0$. Their nearly identical forms serve as a conformation of the hypothesis discussed in Section VI. Figure VII.B.6 illustrates a similar comparison at $y=50$ units above the initial interface, again confirming the remarkable observation of form function 


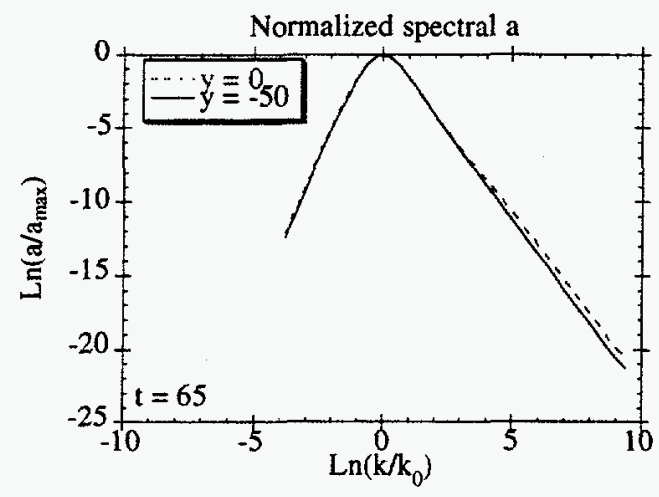

(a)

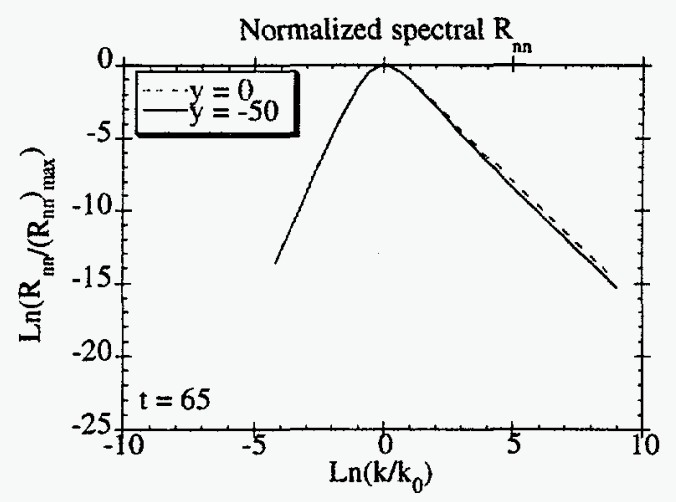

(c)

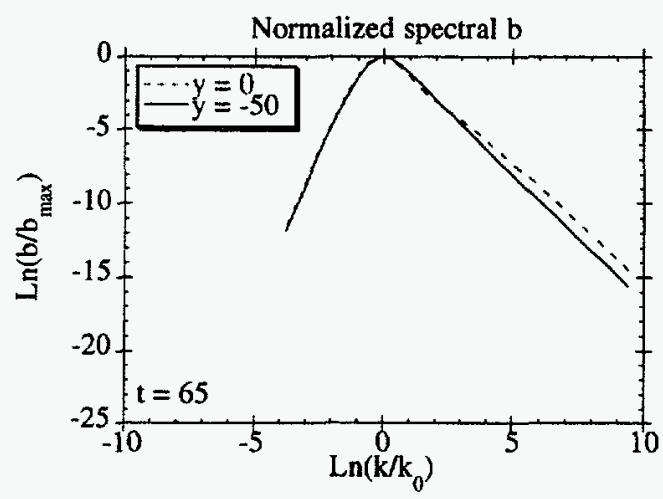

(b)

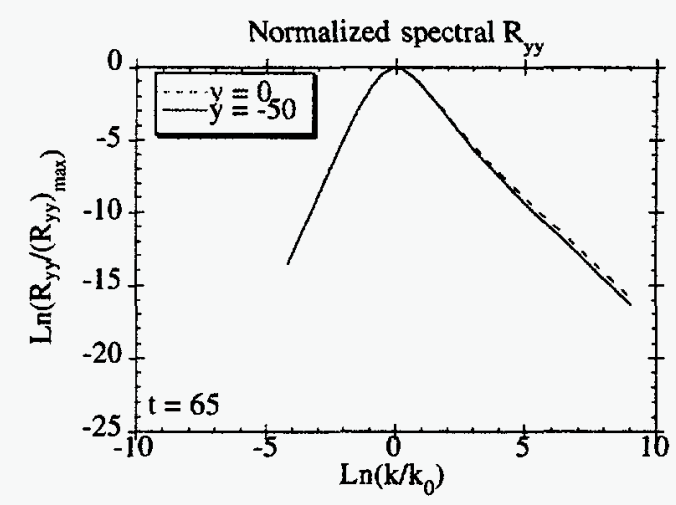

(d)

Fig. VII.B.5. Comparison of normalized spectra at $y=0$ and $y=-50$ for $t=65$ for the variables: (a) the net mass-flux velocity, (b) the specific volume-density correlation, (c) the contraction of the Reynolds stress tensor, and $(d)$ the $R_{y y}$ component of the Reynolds stress tensor.

decomposition hypothesis described in Section VI. This occurs only because of neglect of molecular effects and surface tension. It should be emphasized that the spectral normalizations to unit magnitude and shift to $\mathrm{k}=\mathrm{k}_{0}$ have occurred over very large and different ranges in these figures, so that their coincidence seems all the more remarkable. Because this behavior has also been observed in free-shear turbulence of a fluid with constant density (Besnard et al., 1990; Clark, 1992) we believe that there is a powerful underlying scaling principle that can be exploited in the systematic analysis of certain turbulence circumstances that are inhomogeneous, anisotropic, and even have large variations in fluid density. 


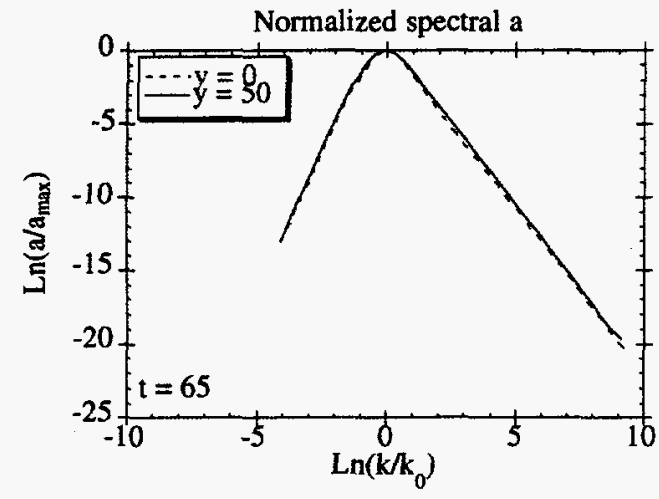

(a)

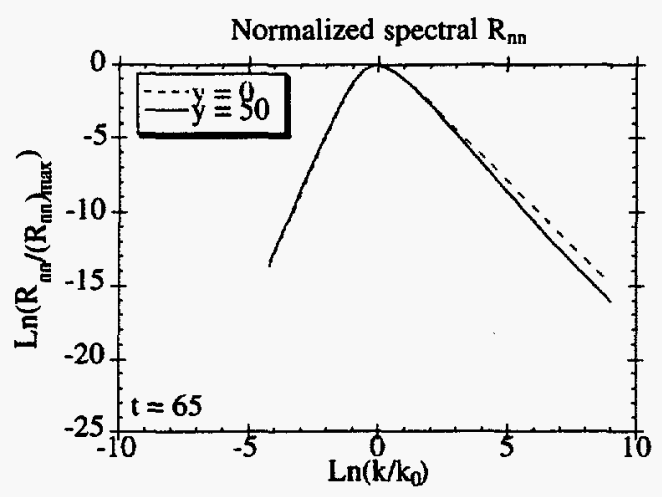

(c)

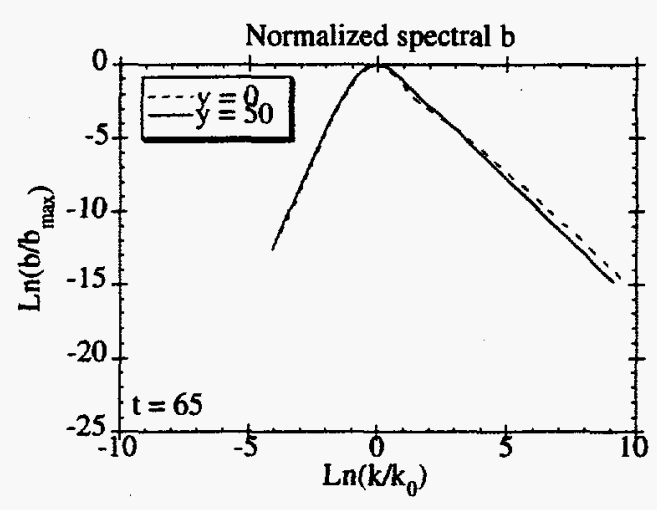

(b)

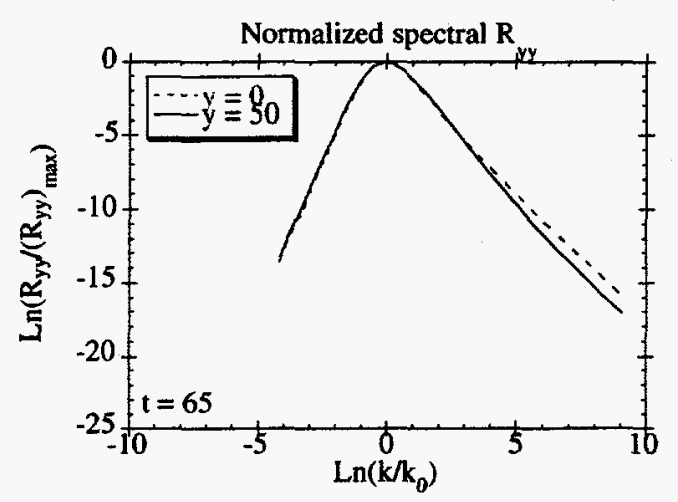

(d)

Fig. VII.B.6. Comparison of normalized spectra at $y=0$ and $y=50$ for $t=65$ for the variables: (a) the net mass-flux velocity, (b) the specific volume-density correlation, (c) the contraction of the Reynolds stress tensor, and $(d)$ the $R_{y y}$ component of the Reynolds stress tensor.

Figure VII.B.7 illustrates the nature of $\mathrm{k}$ shift that has been employed in getting the preceding plots of spectral form functions. In this figure we show the reciprocal of the wave number at which each spectrum has a maximum as a function of position across the TMZ. The ordinate is thus a length scale, which relates the length scale proportional to $K^{3 / 2} / \varepsilon$ that occurs in singlepoint K- $\varepsilon$ turbulence transport models. Structural details in these length-scale profiles have their origin in the initialization for calculations where we only initialize the one cell containing the original fluid-fluid interface, which is far from self-similar. These minor details, once established during the early-time adjustments to self-similarity, are captured and maintained by the achievement of that self-similarity, indeed confirming the attainment of that state. Of more substance, however, are the overall forms of these plots. The length scales for $a(k)$ and $b(k)$ more-or-less decrease towards the edges of the mixing layer, whereas the length scales for $R_{n n}(k)$ 


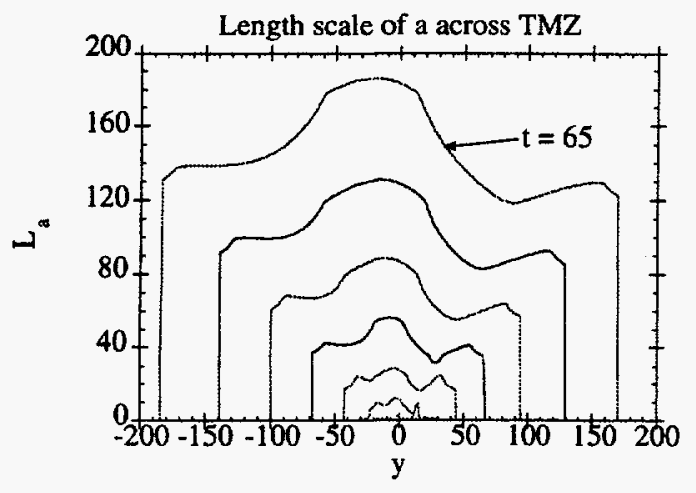

(a)

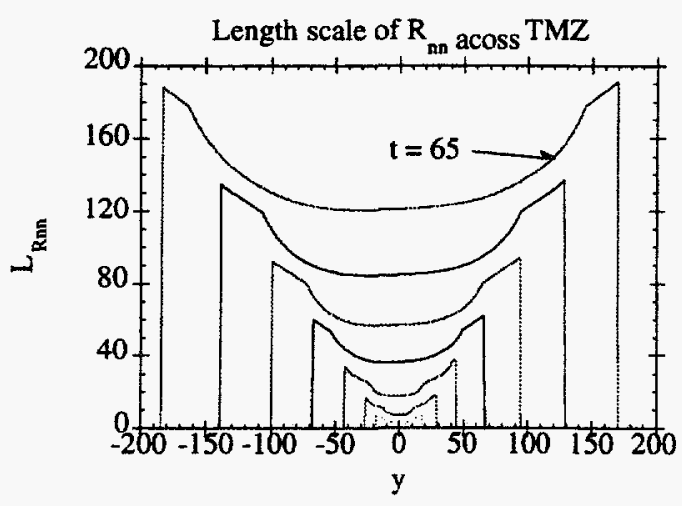

(c)

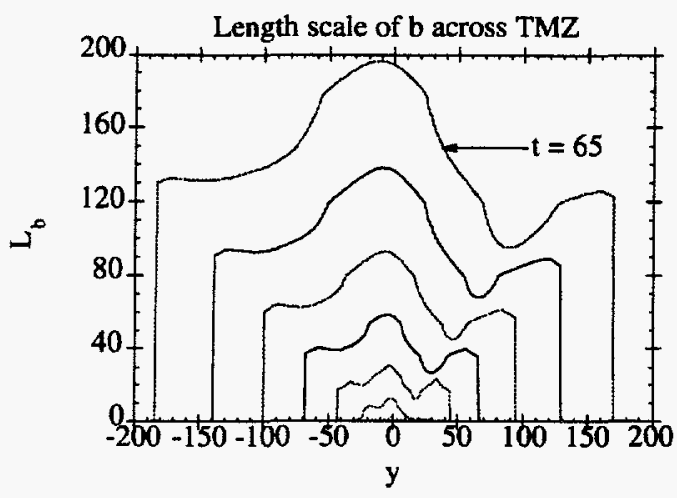

(b)

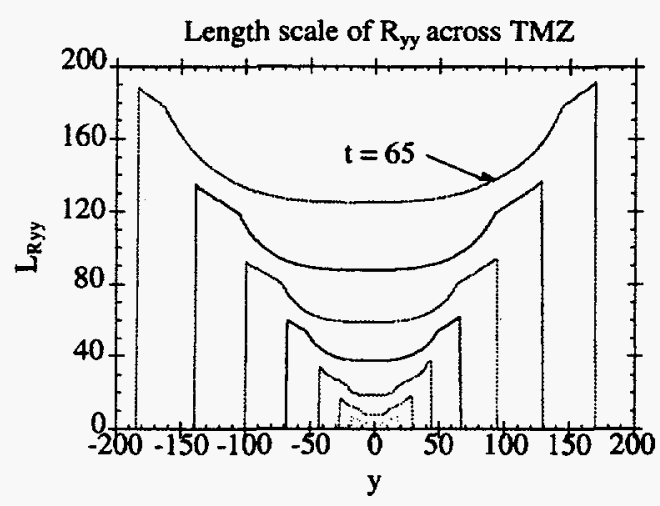

(d)

Fig. VII.B.7. Profiles of the length scales across the $T M Z$ for $t=5,15,25,35,45$, 55, and 65: (a) the net mass-flux velocity, (b) the specific volume-density correlation, (c) the contraction of the Reynolds stress tensor, and $(d)$ the $R_{y y}$ component of the Reynolds stress tensor.

and $R_{y y}(k)$ increase. The basic reason for the laterally increasing length scales for Reynolds stress can be traced to the nonlocal source. This identification is confirmed by contrasting with the results for a local source term; see Section VII.D, which contains figures that show a lateral decrease in scale for $R_{n n}(k)$ and $R_{y y}(k)$. The significance of this effect for single-transport modeling is the lack of proportionality among length scales, for which we obtain remediation by allowing for more than one scale to arise in the moment derivations. (This generalization becomes even more significant in the nonself-similar response to rapid transients of drive.)

Self-similarity also occurs in physical space, as shown in Fig. VII.B.8, again confirming the hypothesis stated in Section VI. The initialization of $b(k)$ at $y=0$ is seen as the spike at $t=0$. Lateral "wings" on the Reynolds-stress profiles arise as a consequence of the nonlocality of the creation term. 


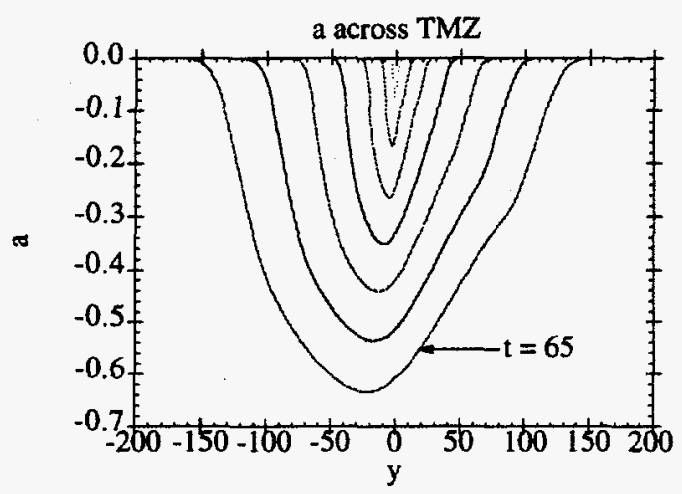

(a)

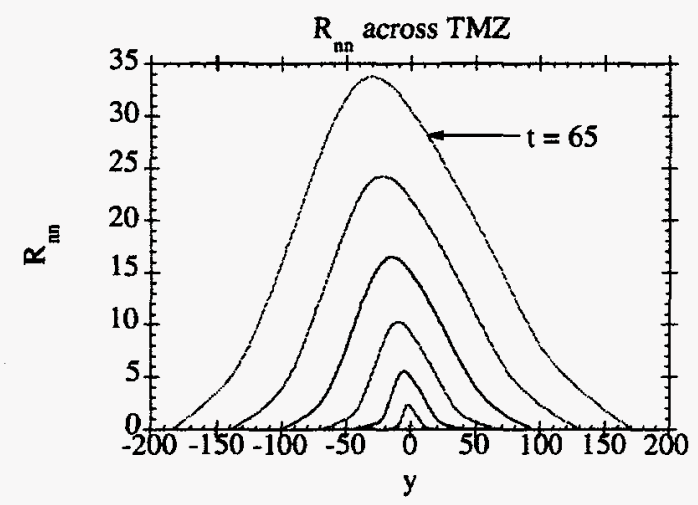

(c)

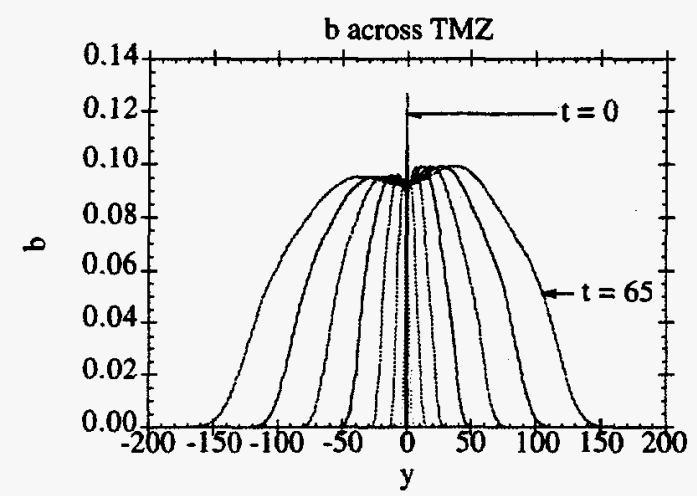

(b)

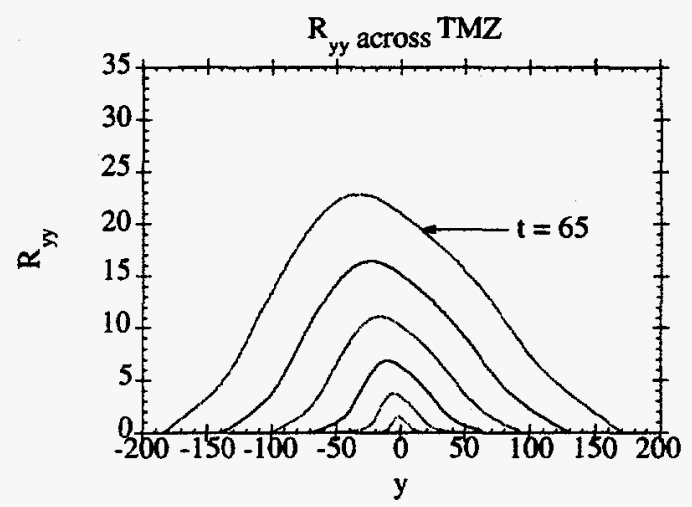

(d)

Fig. VII.B.8. Profiles of spectrally integrated variables across the TMZ for $t=5,15,25,35$, 45, 55, and 65: (a) the net mass-flux velocity, (b) the specific volume-density correlation, (c) the contraction of the Reynolds stress tensor, and $(d)$ the $R_{y y}$ component of the Reynolds stress tensor.

Figure VII.B.9 describes the numerical results in terms of observables that can be compared with experimental data. The principal data are the depth of penetration of heavy fluid into light, $h_{2}$, of light fluid into heavy, $h_{1}$, and the variations of relative concentration between these edges of the TMZ. In this figure we describe the concentration profiles in terms of mean density, $\bar{\rho}$, together with the mass fraction and volume fraction of the heavier material. As pointed out by a Russian experimental group (Kucherenko et al., 1991), the most convenient way to illustrate the profile shape is to plot volume fraction as a function of the reduced variable

$$
\Xi \equiv \frac{\mathrm{y}-\mathrm{y}_{0.1}}{\mathrm{y}_{0.9}-\mathrm{y}_{0.1}}
$$




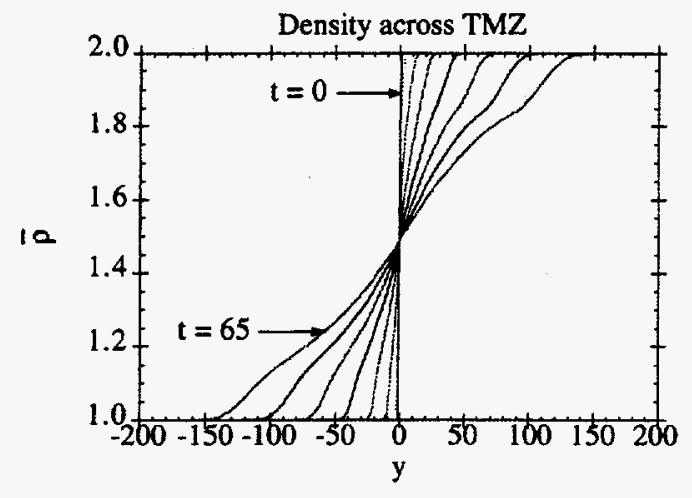

(a)

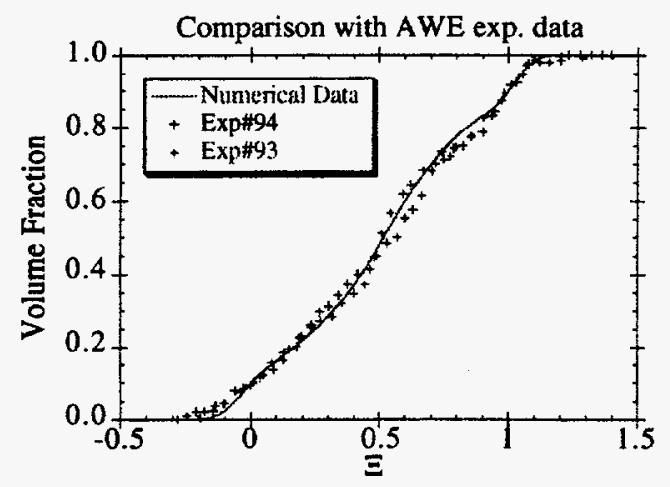

(c)

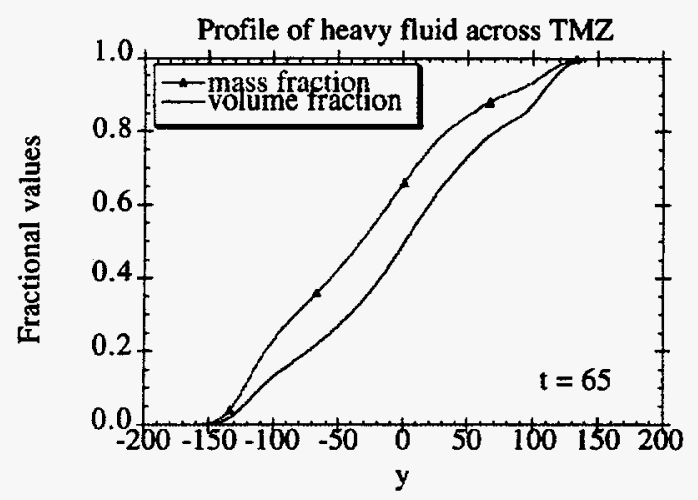

(b)

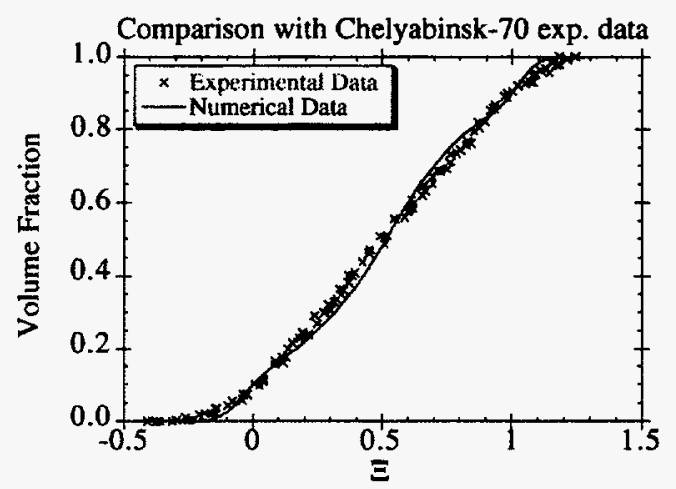

(d)

Fig. VII.B.9. (a) Profiles of density across the TMZ for $t=0,5,15,25,35,45,55$, and 65 . (b) Mass and volume fractions of the heavy fluid across the TMZ at $t=65$. (c) Comparison of numerical results with AWE experimental data [Smeeton and Youngs, 1987 (Fig. 24 and 25)]. (d) Comparison of numerical results with Chelyabinsk-70 experimental data [Kucherenko et al. 1991 (Fig. 11)].

where $y_{0.1}$ and $y_{0.9}$ are the positions at which the volume fraction is 0.1 and 0.9 , respectively. The excellence of agreement with both the British and Russian experimental data is not as trivial as the simple structure of the profiles would suggest. Two-field transport theory also achieves this degree of agreement (see Steinkamp, 1995), but the single-point turbulence transport calculations using the BHR model has great difficulty in matching this simple profile of volume fraction across the TMZ without resorting to an ad hoc modification of the transport equation for the mass flux.

The plots as functions of $\Xi$ in Fig. VII.B.9 exhibit the structure but preclude the observation of behavior for $h_{1}(t)$ and $h_{2}(t)$. These quantities are shown in Fig. VII.B.10 as functions of time and of the similarity variable, 


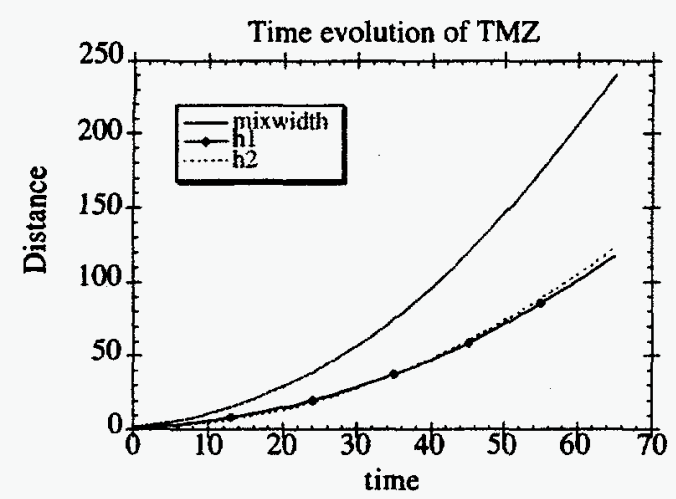

(a)

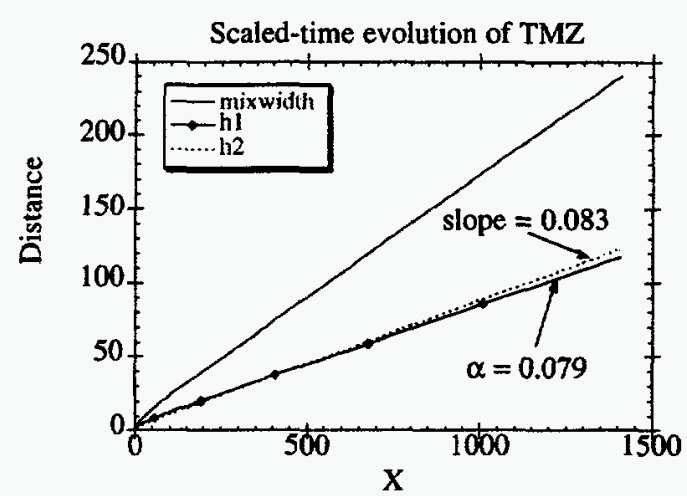

(b)

Fig. VII.B.10. Spikes, $h_{2}$, and the bubbles, $h_{1},(a)$ as functions of time and $(b)$ as functions of the similarity variable, $X$.

$$
\mathrm{X}=\mathrm{At} \mathrm{gt}^{2}
$$

From an experimental viewpoint, the evolutions of $h_{1}$ and $h_{2}$ depend on how the edges of the TMZ are located. The same comment applies to the calculation results. As shown by the Russian experimentalists (see Section VII.D), edge criteria at volume fractions of 0.01 and 0.99 (fine-edge criteria) result in much larger values of $h_{1}$ and $h_{2}$ (and of $h_{2} / h_{1}$ ) than criteria at volume fractions of 0.1 and 0.9 (coarse-edge criteria). They also show and we confirm that the ratio $h_{2} / h_{1}$ converges very slowly to asymptotic value, being highly dependent on early-time approach rate to self-similarity. They mitigate this difficulty by giving instead data for

$$
\frac{\mathrm{dh}_{2}}{\mathrm{dt}} / \frac{\mathrm{dh}_{1}}{\mathrm{dt}}
$$

which is calculated as the ratio of slopes in Fig. VII.B.10(b) and equals $h_{2} / h_{1}$ at late stages in the self-similarity. In Section VII.D the calculated results are shown to lie a bit low for a nonlocal source and a bit high for a local source when compared with the data.

Self-similarity in our case means that the spreading of the TMZ can be characterized by

$$
\mathrm{h}_{1}=\alpha \mathrm{At} \mathrm{gt}^{2}
$$

This expression for the height of the bubble envelope was empirically formulated from experiments by Read (1984) and Youngs (1989). There is experimental uncertainty to the value 
of the coefficient, $\alpha$, with quoted values anywhere from 0.02 to 0.3 and greater. Nikiforov (personal communication, 1994) speculates that the large range reflects the effects of miscibility of the fluids. For immiscible fluids there seems to be a fairly broad consensus that $\alpha=0.065 \pm 0.01$. Our calculated value, $\alpha=0.079$, is slightly higher than the currently accepted range.

An issue of some interest for our spectral equations is the rate of return to isotropy. Because the process is nonlocal in wave-number space and a thorough investigation is beyond the scope of this study, we have not examined the process in detail. One point that can be mentioned is illustrated in Fig. VII.B.11, which shows the degree of anisotropy in the base-case calculation. As plotted versus wave number, $R_{n n}(k)$ and $R_{y y}(k)$ look nearly the same, whereas the spectrally integrated quantities plotted as a function of $\mathrm{y}$ demonstrate that there is a significant difference between the two quantities. Note that if $R_{y y}=R_{n n}$, then $R_{x x}=R_{z z}=0$, and the behavior is completely anisotropic; the interpenetration is entirely "ordered." If $R_{y y}=R_{n n} / 3$, then the three diagonal components of the Reynolds stress are equal and the configuration is completely isotropic ("disordered"). At low wave numbers the former condition prevails; at high wave numbers the complete isotropy is approached. Thus the turbulence is created at low wave numbers in primarily anisotropic (ordered) forms and becomes progressively more disordered at any fixed wave number as the TMZ grows and the spectrum shifts to the left. Interpenetration at the large scales (low wave numbers) is thus predominantly wave-like (hyperbolic, as in multifield flow), whereas at the finer scales (high wave numbers) the interpenetration becomes predominantly diffusive (parabolic, as in the more classical circumstances of "turbulence").

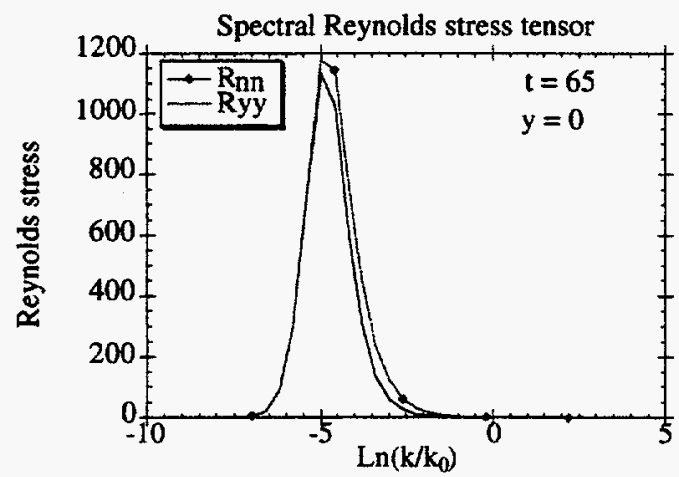

(a)

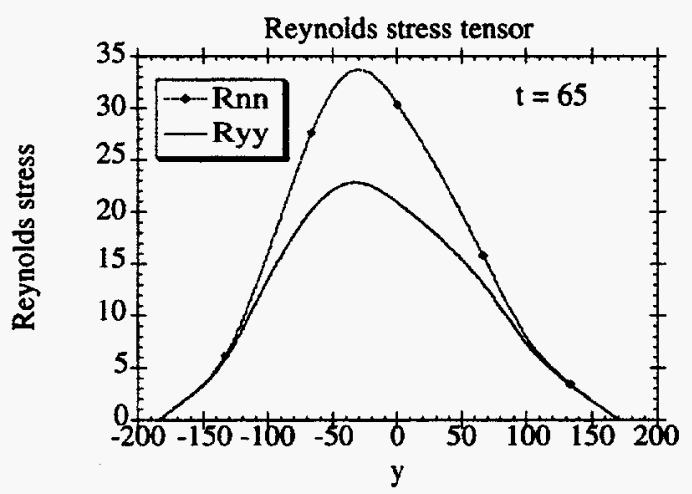

(b)

Fig. VII.B.11. Comparison of the trace of the Reynolds stress tensor with $R_{y y}(a)$ at the centerline at $t=65$ and $(b)$ in spectrally integrated form across the TMZ. 
We now describe some calculations that depart in one or another respect from the base case. In the first of these variants, we investigated the consequences of blocking the cascade flux of $b(k)$ at a high wave number. Spectral integrals of $b(k)$ are shown in Fig. VII.B.12. With zero spectral flux, the cascade flux is conservative and the integral now includes the passive parts of the spectrum that pile up at large wave numbers. The representation is a crude approximation to the behavior that would be expected from the presence of a smallest attainable scale, corresponding to a particle size that is not further subdividable. Whereas the $\mathrm{C}_{\mathrm{fb}}$ term describes an inverse transfer to progressively larger scales, the cascade to small scales shows turbulence effects in tearing down the clumps. With a large Schmidt number, the latter process would be expected to lead to the $\mathrm{k}^{-1}$ spectral behavior of a passive scalar for those clump scales whose wave numbers exceed the viscous cutoff. We have not investigated such circumstances. All of these considerations require an extensive program of investigation that lies far beyond the scope of this present study. Figure VII.B.12 shows that the added contribution to the spectral integral is noticeable, especially for the spatial positions (near the original interface) for which direct cascade has been proceeding long enough for a high-wave-number buildup. The most significant feature of this calculation is the negligible effect of blockage on the rest of the variables, not shown here because the differences are simply not visible on the plots. Thus the concept of a passive part for the $b(k)$ spectrum is embodied; in that range of wave numbers the creation of $a(k)$ is essentially balanced by the large drag of the small-scale clumps, in a range of $a(k)$ that is in any case so small (from $\mathrm{k}^{-7 / 3}$ scaling) that its effect is negligible. The configurational value of b in the Fig. VII.B.12 is given by (Besnard et al. 1992) as

$$
\frac{\alpha_{1} \alpha_{2}\left(\rho_{1}-\rho_{2}\right)^{2}}{\rho_{1} \rho_{2}}
$$

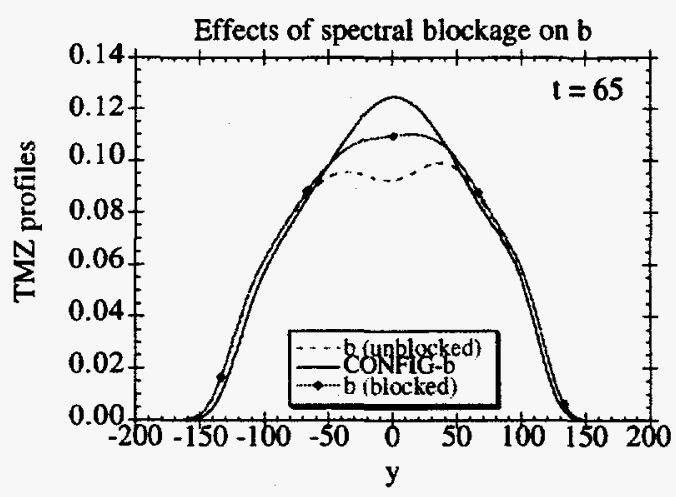

Fig. VII.B.12. Profile of $b$ across the TMZ for the case of a spectrally unblocked calculation and a spectrally blocked calculation compared with the configurational calculation of $b$, at $t=65$. 
The transported values of $b(k)$ follow the configurational form rather closely, being less near the center and somewhat higher in the lateral regions of the TMZ, principally as a result of diffusion in the transport equation, which is conservative in physical space. Some implementations of single-point turbulence transport equations (e.g., the BHR model) into computer codes use a purely configurational form for $b$, and our results are consistent with that procedure. Having demonstrated the close relationship between transported $b$ and configurational $b$, we wonder why not use purely the configurational form. The answer is in two parts.

- There is no configurational form for spectral $b(k)$, for which the distribution across wave-number space is needed for the spectral analysis.

- In the presence of phase transitions or molecular diffusion between the two fluids, the transport equation can be directly modified to include these effects, together with the resulting decay of $b(k)$ ultimately to zero. In the examples described here, however, we have assumed that the rate of these extra processes is zero, so that $C_{d b}=0$.

With a sudden complete reversal of the body-force acceleration (from $g=-1$ to $g=+1$ ), the zone is expected to de-mix back to its original state with a sharp interface. As pointed out by Nikiforov (personal communication, 1994), single-point transport equations in a finite difference implementation have difficulty in calculating this process. We confirm that difficulty and have identified the problem. De-mixing is highly dissipative of energy. In contrast to mixing, in which clumps of a material do not collide with others of the same material, de-mixing is highly collisional within the field of each material. The inelastic collisions involve much more microphysics than our current model contains, in particular splash with possible entrainment of the other material. Two-field models calculate de-mixing with deceptive ease in the circumstance of no splash (the "splat-and-stick" limit). When $\alpha_{1}$ or $\alpha_{2}$ has increased back to 1.0, the calculation holds the volume fraction constant thereafter, and the incompressibility condition for that field ensures that the velocity comes at once to rest, thereby accomplishing a continuous dissipation of collisional energy as each fluid returns to its pure state. This matter is discussed more extensively by Steinkamp (1995) for the two-field calculations in this limiting mode of demix representation.

\section{Numerical Results for a Rapid Transient}

We have not yet investigated the possible inelastic collisional interactions within a fluid and their appropriate dissipative representation. The issue of particular concern to our developments is the question of self-similarity during the rapid-transient phase of turnaround induced by acceleration reversal. The principle issue concerns the variations of $a(k)$. The other variables, $\mathrm{b}(\mathrm{k}), \mathrm{R}(\mathrm{k})$, and $\mathrm{Ryy}(\mathrm{k})$ have much smaller transient response. With the reversal of $\mathrm{g}$ there is likewise an instantaneous reversal of $\partial \mathrm{p} / \partial \mathrm{y}$. The differential acceleration driver to $\mathrm{a}(\mathrm{k})$ thus 
reverses; for a brief period of adjustment that term works in concert with the drag terms towards reversal of the sign of $a(k)$; in this regard the entire spectrum of $b(k)$ becomes active. For the acceleration stage, the pressure and density gradient terms in Fig. VII.C.1(a) balance the drag terms at high wave numbers, resulting in negligible changes in $a(k)$, vanishingly small $\partial a(k) / \partial t$. Immediately after the acceleration reversal at $t=65$, the pressure gradient term changes sign, resulting in a relatively larger change of a(k) for the high wave numbers. In this respect we say that the high wave number portion of the $b(k)$ spectrum has changed from passive to active.

When a(k) reverses sign, the mass flux becomes counter-gradient to the density variation; the two driver terms in the evolution equation for $a(k)$ now work in opposition to one other. To further illustrate this point, we see that the pressure gradient term and the density gradient term both counter the drag terms in Fig. VII.C.1(a), whereas in Fig. VII.C.1(b), immediately following the acceleration reversal, the density gradient term counters not only the two drag terms, but also the pressure term. (As pointed out by Youngs (personal communication, 1993), this opposition is necessary for describing his de-mixing experiments: differential acceleration works to compact the zone while density-gradient turbulence flux tends to preserve its dispersal.)

Although the brief opposition of $\mathrm{a}(\mathrm{k})$ and $\partial \mathrm{p} / \partial \mathrm{y}$ during the slowdown parts of turnaround give a negative source to the components of $R_{\mathrm{ij}}(\mathrm{k})$, the net effect is small. For the calculation of turnaround results, we used the spectrally blocked version of the base-case conditions described for Fig. VII.B.12, allowed the attainment of self-similar mixing, reversed $g$ (from -1 to +1 ) at $t=65$, and recorded the turnaround until its more-or-less completion at $t=80$. The transient phases of structure for $\mathrm{a}(\mathrm{k})$ are shown in Fig. VII.C.2(a). There is a clear departure from the selfsimilarity during the process. The large scales (small wave numbers) exhibit a more immediate

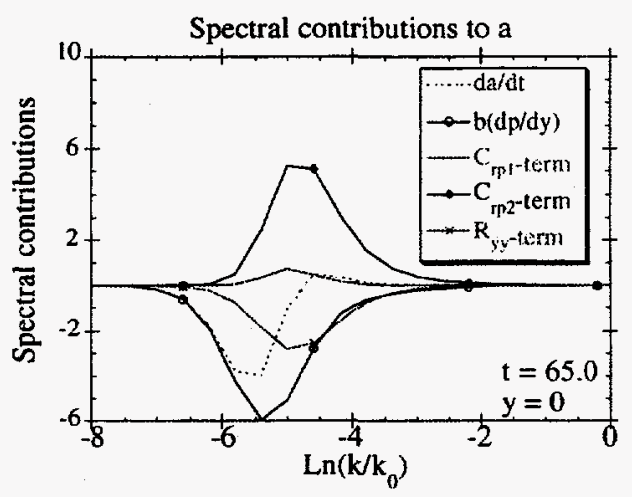

(a)

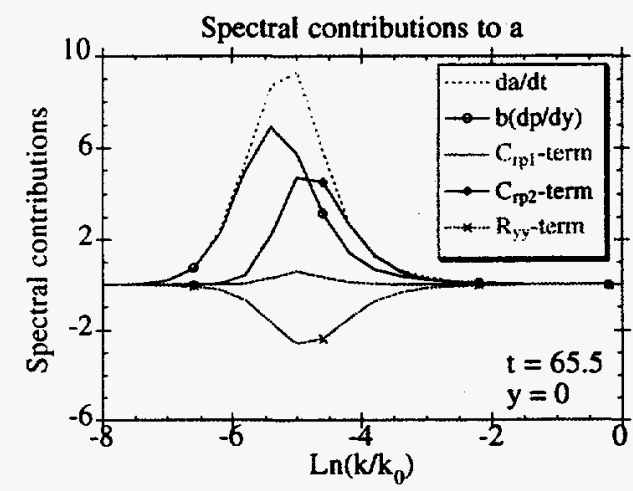

(b)

Fig. VII.C.1. Spectral contributions of the dominant sources and sinks to the a equation for: (a) mixing $(g=-1)$, and (b) demixing, immediately after acceleration reversal $(g=1)$. 
response than the small scales (at large wave numbers). A simple amplitude-modulated function with slowly shifting position in $\mathrm{k}$ space for its maximum is not strictly what occurs; although, in effect, single-point model equations assume this.

Figure VII.C.3 shows that, although the de-mixing process that follows flow reversal cannot be self-similar, the spectral forms as shown in Fig. VII.C.3 return to almost precisely those that were present before the acceleration reversal. The implications are important for momentintegral derivations of single-point transport equations: almost-universal forms occur in much wider circumstances than just those that are self-similar.

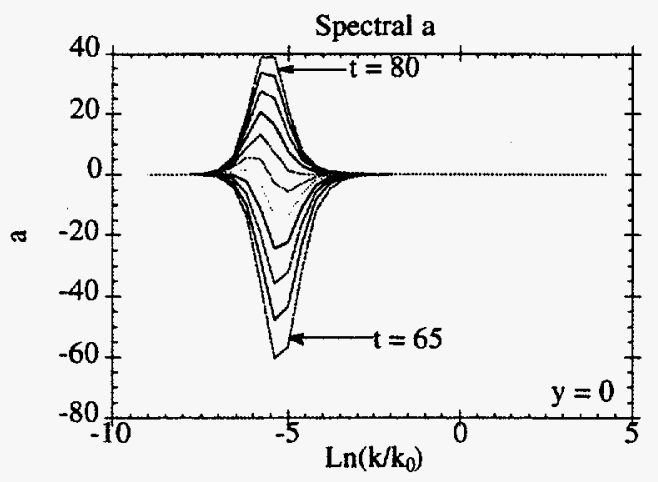

(a)

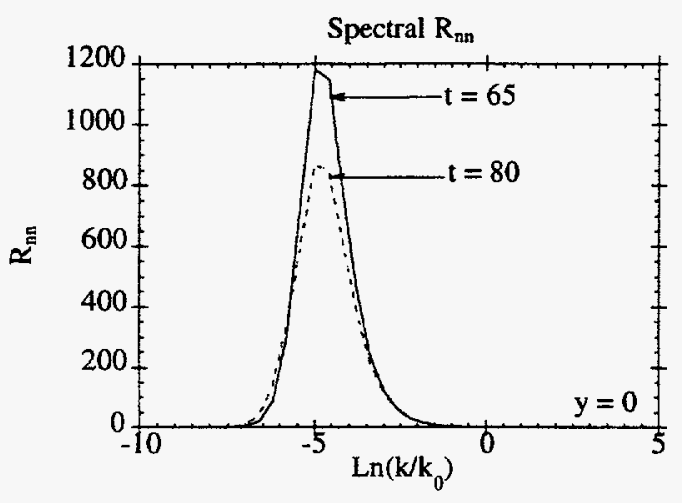

(c)

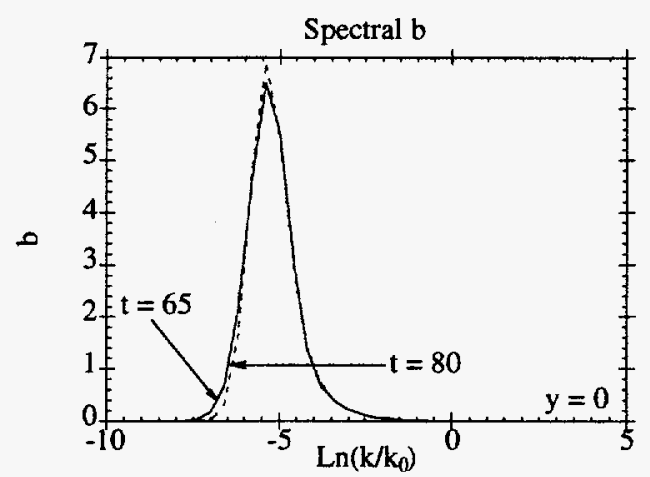

(b)

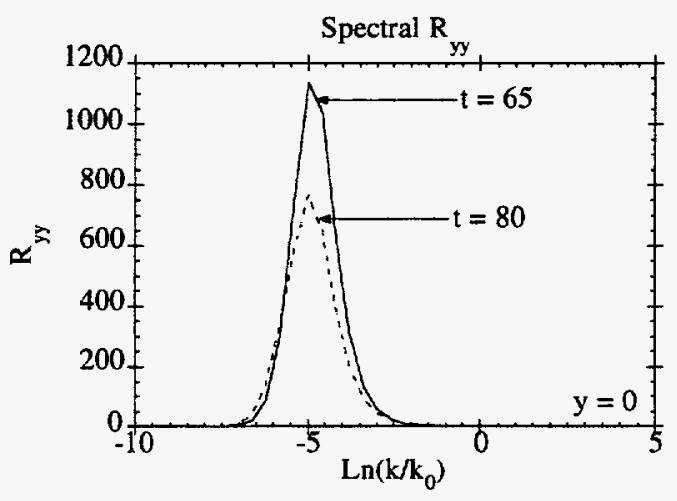

(d)

Fig. VII.C.2. Spectra at times $t=65$ and 80 , at the position of the initial fluid interface, for: (a) the net mass-flux velocity, a (including plots for every $\Delta t=1.5$ ), (b) the specific volumedensity correlation, $b,(c)$ the contraction of the Reynolds stress tensor, $R_{n n}$, and (d) the $R_{y y}$ component of the Reynolds stress tensor. 


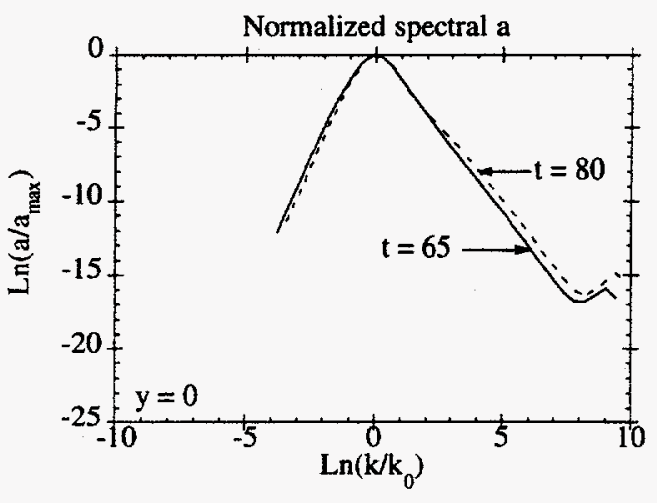

(a)

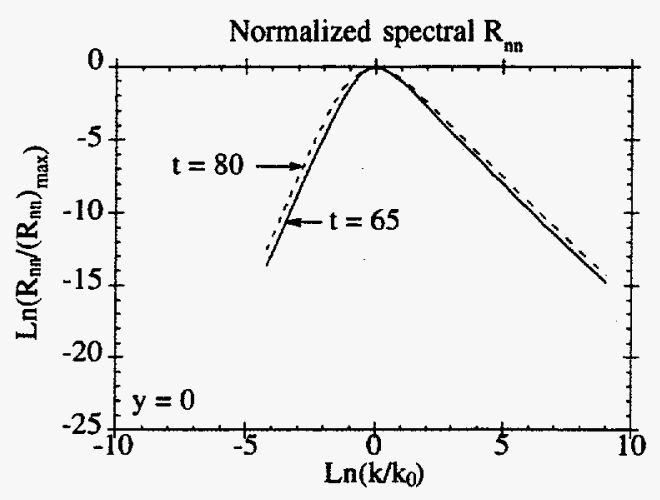

(c)

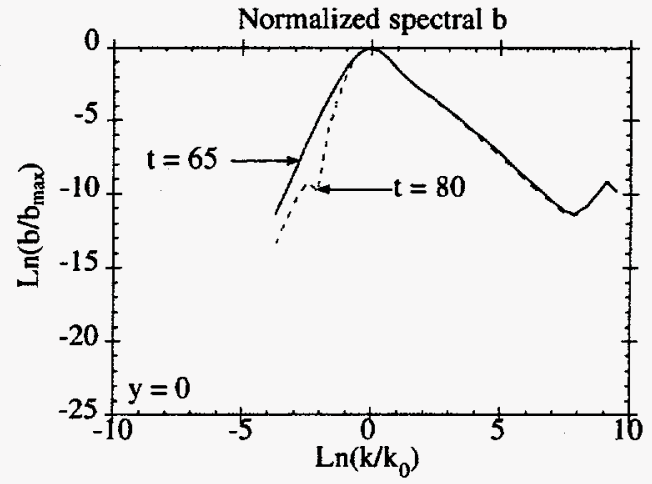

(b)

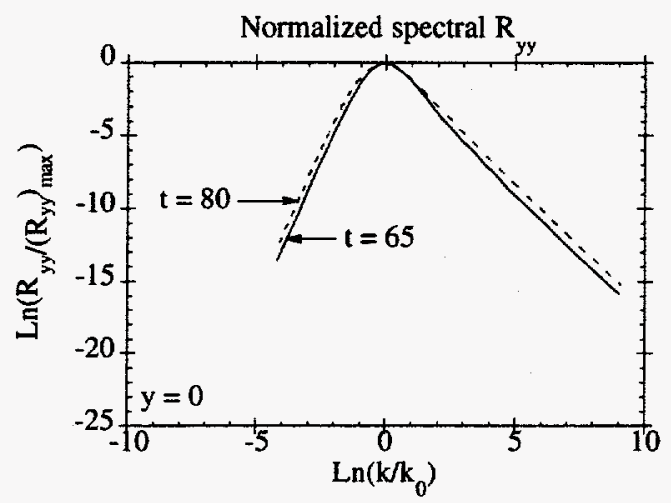

(d)

Fig. VII.C.3. Normalized spectra for $t=65$ and 80, at the position of the initial fluid interface, for: (a) the net mass-flux velocity, $a,(b)$ the specific volume-density correlation, $b,(c)$ the contraction of the Reynolds stress tensor, $R_{n n}$, and, (d) the $R_{y y}$ component of the Reynolds stress tensor.

Variations of spectrally integrated quantities across the TMZ are illustrated in Fig. VII.C.4. Figure VII.C.4(a) shows that the heavier clumps of fluid respond more quickly to gravity reversal than the light clumps. The most startling observation is in Fig. VII.C.4(b), which shows negative values of $b$ where the heavier fluid is falling back into itself.

The anomaly is also seen in Fig. VII.C.5, in which the average density exceeds 2.0 at this fall-back locality. In the two-field calculations of Steinkamp (1995), this impossibility is avoided by the dissipative technique described above for one possible type of mixing. The same "stick-and-splat" fix technique could also be employed here, but the topic requires extensive consideration and discussion for more general de-mixing circumstances, which is beyond the scope of this present study. 


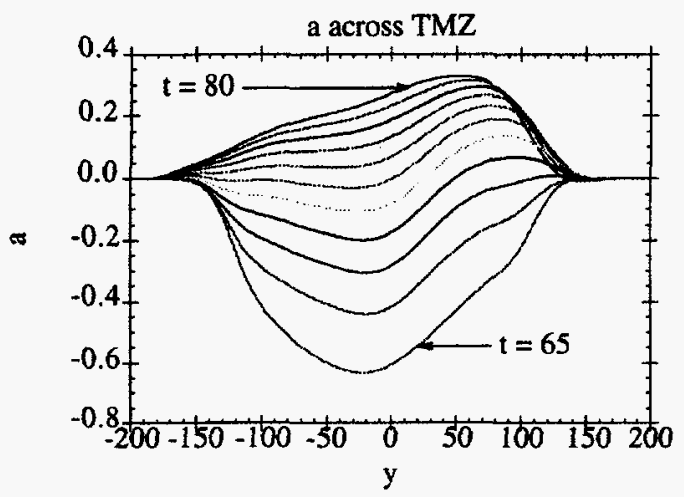

(a)

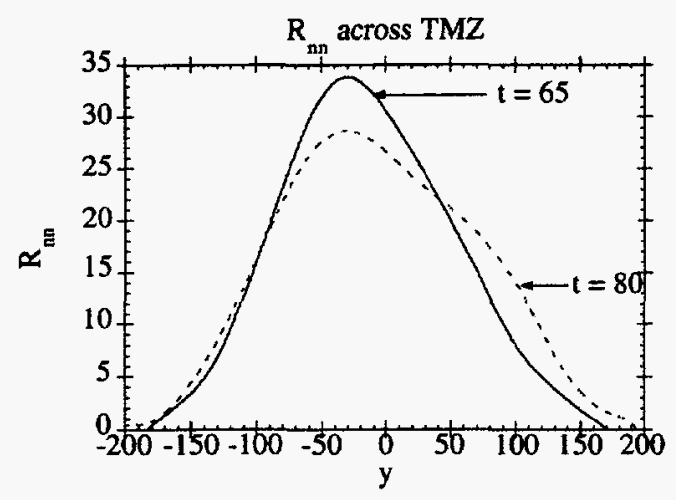

(c)

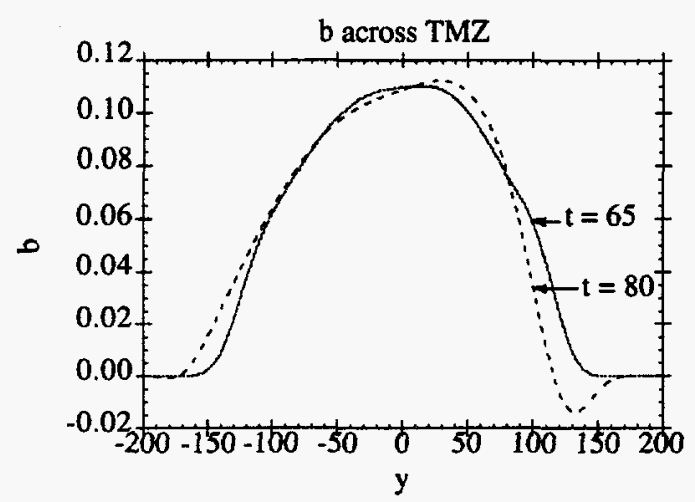

(b)

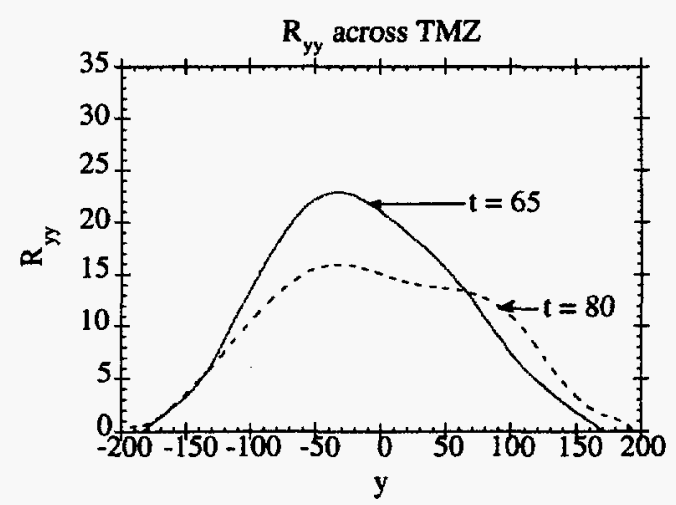

(d)

Fig. VII.C.4. Profiles of spectrally integrated variables across the TMZ for $t=65$ and 80 of (a) the net mass-flux velocity, a (including plots for every $\Delta t=1.5$ ), (b) the specific volumedensity correlation, $b$, (c) the contraction of the Reynolds stress tensor, $R_{n n}$, and $(d)$ the $R_{y y}$ component of the Reynolds stress tensor.

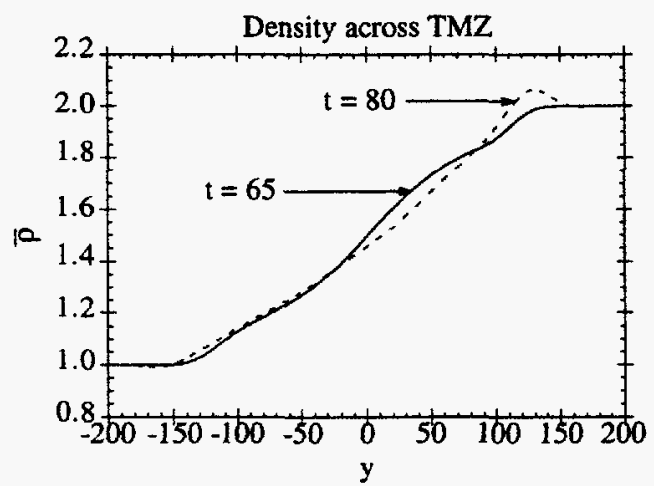

Fig. VII.C.5. Profiles of density across the TMZ for $t=65$ and 80 . 
The base case calculation without spectral blockage was also performed for the mixing of two fluids with a larger density ratio, $\rho_{2} / \rho_{1}=5.0$. The results exhibit essentially the same attainment of self-similarity, with spectral exponents corresponding to those in Fig. VII.B.2.

\begin{tabular}{|c|c|c|}
\hline Quantity & $\begin{array}{c}\text { Low-k } \\
\text { exponent }\end{array}$ & $\begin{array}{c}\text { High-k } \\
\text { exponent }\end{array}$ \\
\hline $\mathrm{R}_{\mathrm{ij}}(\mathrm{k})$ & 3.99 & -1.74 \\
\hline $\mathrm{b}(\mathrm{k})$ & 3.87 & -1.71 \\
\hline $\mathrm{a}_{\mathrm{i}}(\mathrm{k})$ & 3.92 & -2.37 \\
\hline
\end{tabular}

TABLE VII.C.1. Power law behavior for both the low and high wave numbers for the transported spectral variables.

For the case with a density ratio of 5.0, as in Fig. VII.B.3, the ordering of exponent values in Table VII.C.1 for low wave numbers is the same and for the same reasons having to do with the effects of $\mathrm{C}_{\mathrm{fb}}$. For high wave numbers we see again the large contrast between the cascadedominated behavior of $R(k)$ and $b(k)$, for which $n \approx-5 / 3$ and the drag-dominated behavior of $a(k)$, for which $n \approx-7 / 3$. The value of $\alpha$, the self-similar growth coefficient for $h_{1}$, is 0.11 , which is appreciably larger than either the base-case result or the experimentally observed result. In addition, $\mathrm{h}_{2} / \mathrm{h}_{1}$ approaches the magnitude 1.08 , which is low.

\section{Local vs. Nonlocal Source Term}

Two intriguing disagreements with experiments are especially noticeable in the results discussed so far (see Table VII.D.1). One is the coefficient for self-similar growth of $h_{1}$, which is somewhat too large, and the other is the asymptotic ratio of $h_{2} / h_{1}$, which is too small. An intriguing clue to remediation of both discrepancies lies in the results described by Figs. VII.D.1-VII.D.5. In those figures we compare the base case with local and nonlocal source terms for $R_{n n}(k)$ and $R_{y y}(k)$. Figure VII.D. 1 shows the large contrast in results. With the local source, density exhibits sharp variations on each side of the TMZ; these are more spread out with the nonlocal source.

The coefficient, $\alpha$, is sharply reduced to the value $\alpha=.042$, while the ratio of penetration depths, $h_{2} / h_{1}$, approaches the much larger value of 1.79 . In both respects the experimental values lie between the extremes. 


\begin{tabular}{|l|c|c|}
\hline Source to $\mathrm{R}_{\mathrm{ij}}$ & $\alpha$ & $\mathrm{h}_{2} / \mathrm{h}_{1}$ \\
\hline local & .042 & 1.79 \\
\hline nonlocal & .079 & 1.05 \\
\hline experiments & $\sim .065$ & $\sim 1.30$ \\
\hline
\end{tabular}

TABLE VII.D.1. Self-similar coefficient, $\alpha$, and the asymmetry of the TMZ for the two numerical runs and experiments.

It thus appears that a more restrictive nonlocal formulation for $\mathrm{Q}\left(\mathrm{y}^{\prime}, \mathrm{y}\right)$ could produce closer agreement with experiments in both respects. The task of deriving such a formulation remains a challenge yet to be addressed.

The spectral behaviors are compared at low and high wave numbers in Figs. VII.D.2 and VII.D.3. At the low wave numbers, there is a significant effect that is discussed in Section VIII. At the high wave numbers there is very little difference between the local and nonlocal formulations. Here we merely observe that the spectrally preserving local source is structurally dominated by the $\mathrm{C}_{\mathrm{fb}}$ effects, so that the exponent is lower than with the nonlocal source, which is not spectrally preserving and indeed tends to increase the exponent at low wave numbers. This complex set of interactions is discussed in more detail in Section VIII.

Figure VII.D.4 is comparable to Fig. VII.B.4, with both showing the evolution towards selfsimilarity at a distance of 50 units below the original interface. The results are quite similar; in both cases the first influence to arrive is that of $a(k)$, which tends to impart $k^{-7 / 3}$ behavior at the high wave numbers, soon to be dominated, however, by the effects of cascade that convert the spectrum to $\mathrm{k}^{-5 / 3}$. At the lower wave numbers the spectrum arrives in completely self-similar

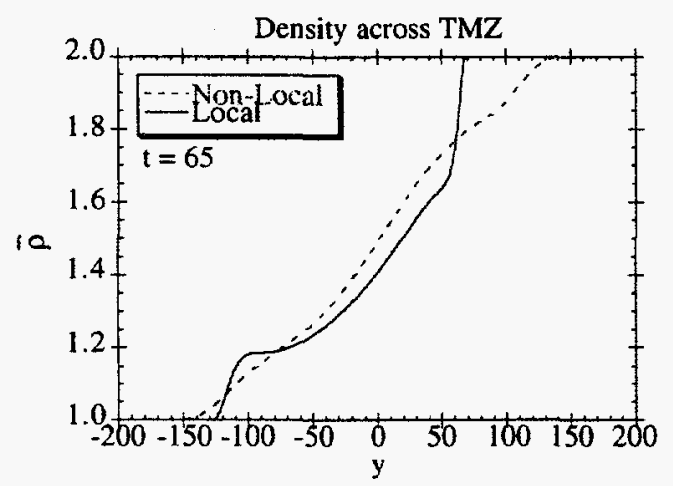

Fig. VII.D.1. Comparison of the density across the TMZ for a local source to $R_{i j}$ and a nonlocal source to $R_{i j}$. 


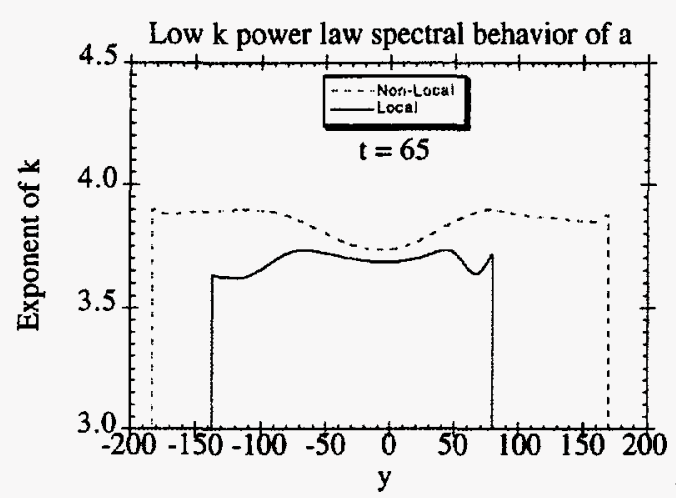

(a)

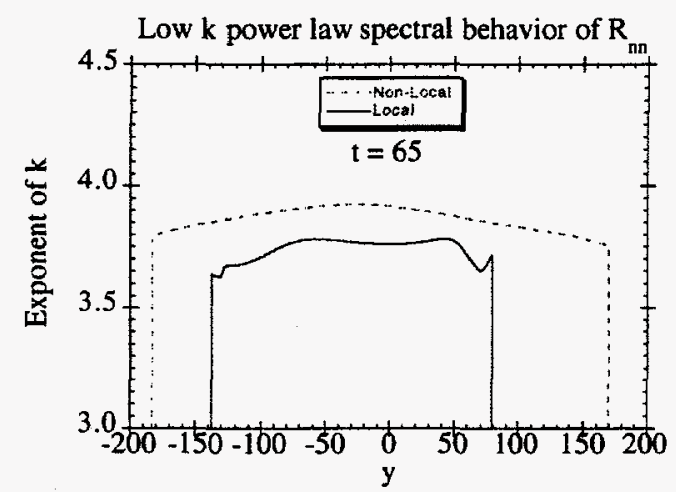

(c)

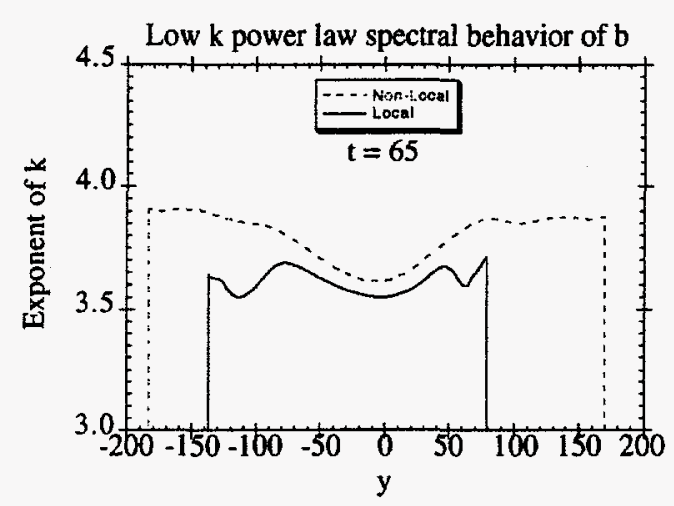

(b)

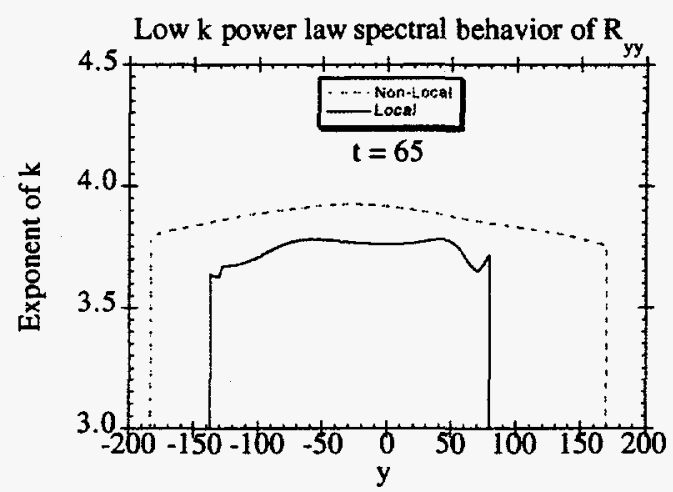

(d)

Fig. VII.D.2. Comparison of the low wave number power law behavior of $k$ at $t=65$ for a local and nonlocal run for: (a) the net mass-flux velocity, $a,(b)$ the specific volume-density correlation, $b,(c)$ the contraction of the Reynolds stress tensor, $R_{n n}$, and, $(d)$ the $R_{y y}$ component of the Reynolds stress tensor.

form. The contrast between local and nonlocal lateral propagation is manifested in slightly less departure from self-similarity at high wave numbers in the local case, especially in the spectra of $R_{n n}$ and $R_{y y}$. The reason is that nonlocal propagation favors more rapid lateral distribution of the larger scales, with corresponding slower lateral distribution of the smaller scales.

This difference in lateral propagation is especially apparent in Fig. VII.D.5, which shows the spatial distributions of scale lengths (reciprocals of wave numbers for the spectral maxima) at a late time in self-similar TMZ growth. Disregarding structural details in the profiles, we notice a significant difference between the local and nonlocal results. For the local source term, the length scales all tend to decrease towards the edges of the TMZ. With a nonlocal source term, 


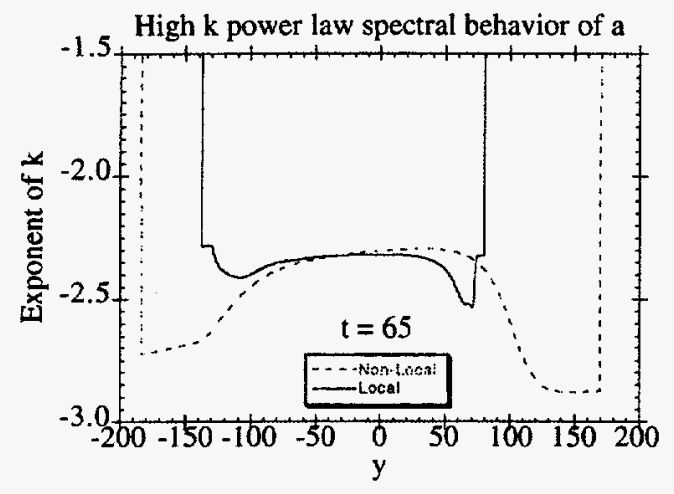

(a)

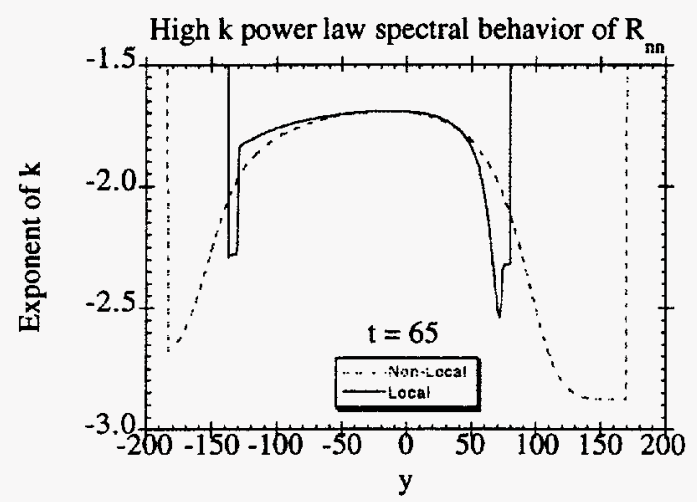

(c)

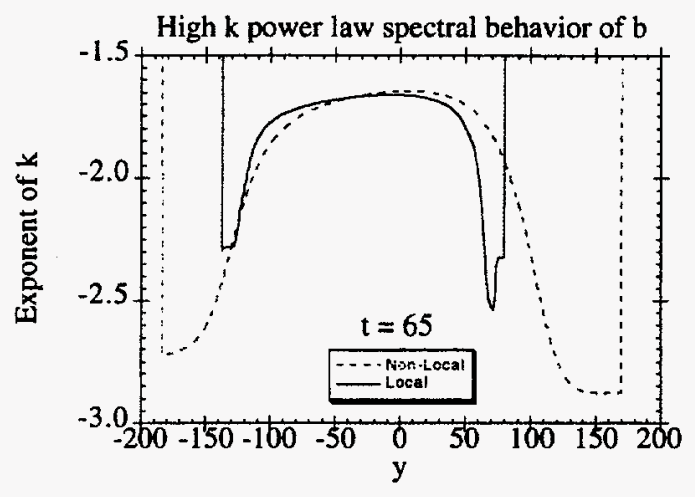

(b)

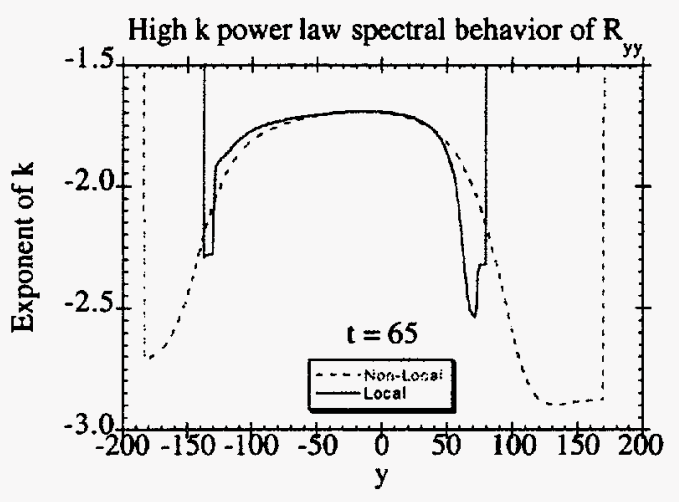

(d)

Fig VII.D.3. Comparison of the high wave number power law behavior of $k$ at $t=65$ for a local and nonlocal run for: (a) the net mass-flux velocity, $a$, (b) the specific volume-density correlation, $b$, (c) the contraction of the Reynolds stress tensor, $R_{n n}$, and, (d) the $R_{y y}$ component of the Reynolds stress tensor.

the profiles for $a$ and $b$ are essentially the same, whereas for $R_{n n}$ and $R_{y y}$ the scales increase significantly towards the edges. It is this feature that especially demonstrates the desired consistency with Rayleigh-Taylor theory, as discussed in Section V.

The issue of $\bar{\rho}$ variations across the TMZ can be put into clearer perspective by further consideration of the experimental observations. Figure VII.D.6 is adapted from the Russian report (Kucherenko et al., 1991) in which they give a very thoughtful analysis of the situation for various different density ratios, $n=\rho_{2} / \rho_{1}$. The principal issue concerns the identification of edge locations for the TMZ for which they use both photographic and $\mathrm{x}$-ray techniques. They define edge positions in terms of a fractional quantity, $\delta$, which designates fraction of purity for the heavier fluid at which the edge is defined to be located. Typical values are $\delta=0.10,0.90$ and 


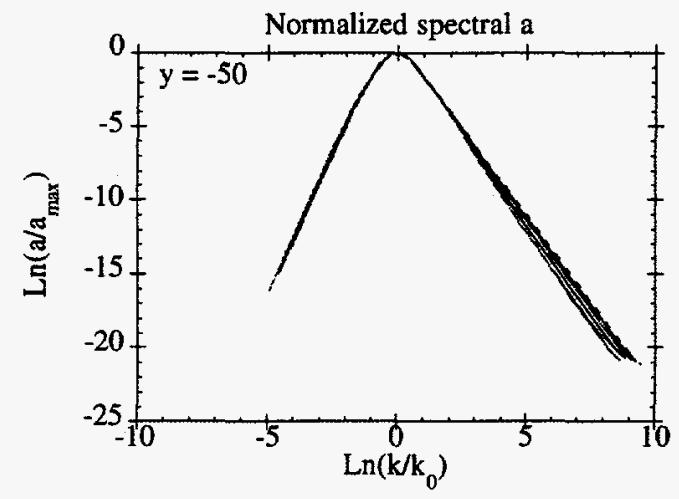

(a)

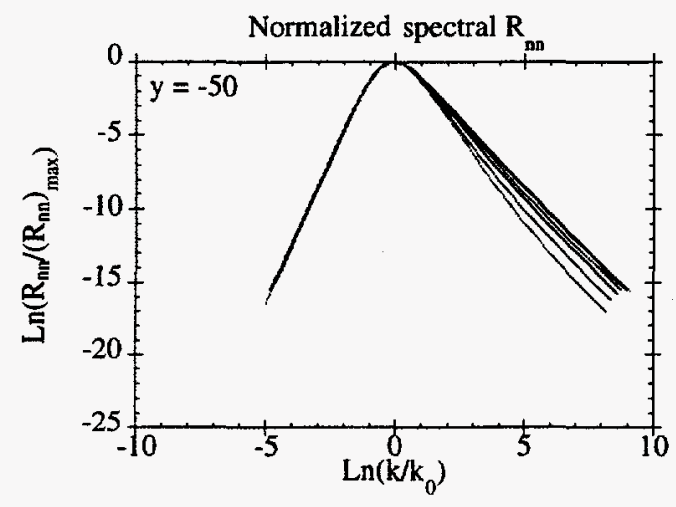

(c)

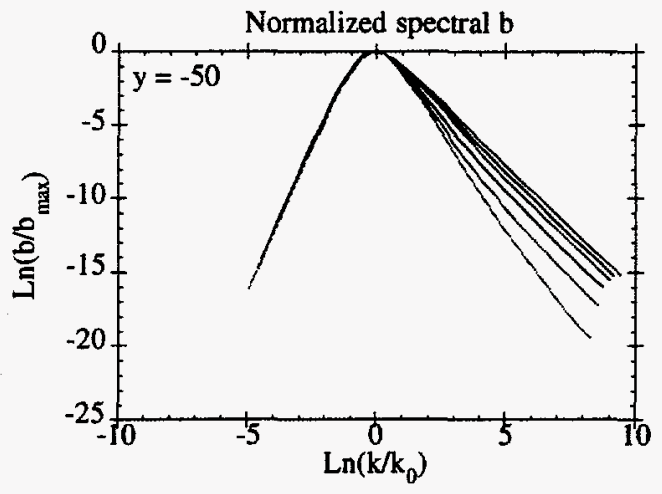

(b)

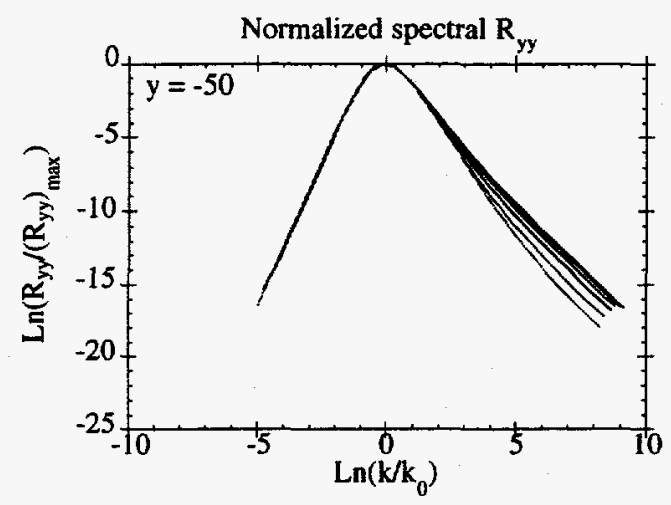

(d)

Fig. VII.D.4. Normalized spectra for $t=30,35,40,45,50,55,60$, and 65 , a distance of 50 units of length below the centerline of the TMZ for: (a) the net mass-flux velocity, $a,(b)$ the specific volume-density correlation, $b,(c)$ the contraction of the Reynolds stress tensor, $R_{n n}$, and, (d) the $R_{y y}$ component of the Reynolds stress tensor.

$\delta=0.01,0.99$ (this last being stated as 0.98 on one of the figures in their report, presumably erroneously). Figure VII.D.6(a) shows the variation of $\bar{\rho}$ across the TMZ for a typical example. Error bars indicate the uncertainty, especially with implications for edge location. With $\delta=0.01$, 0.99 , the distances $h_{1}$, and especially $h_{2}$ depend strongly on how the datum points are connected through the envelope of error bars. With $\delta=0.10,0.90$, the sensitivity is reduced and the ratio, $\mathrm{h}_{2} / \mathrm{h}_{1}$, is considerably smaller. Figures VII.D.6(b) and VII.D.6(c) illustrate the effects of choosing different edge criteria. Combining both Russian and British data, we see in Fig. VII.D.6(d) a considerable scatter in data. The figure also shows the base-case numerical results for both the local and nonlocal sources. Refer to Table VII.D.1 for the values of $h_{2} / h_{1}$. 


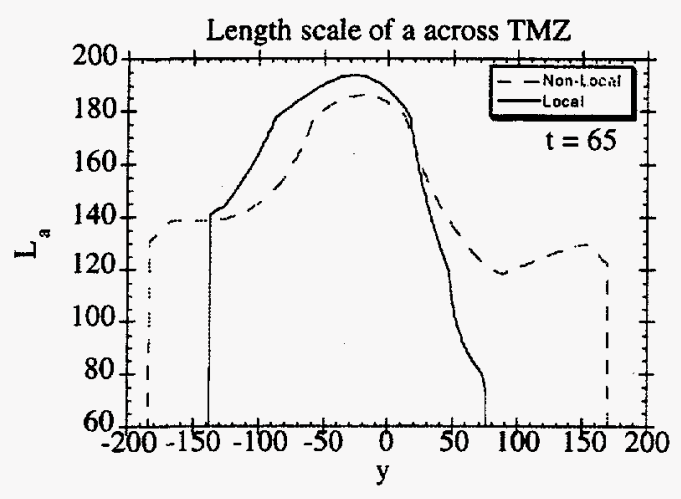

(a)

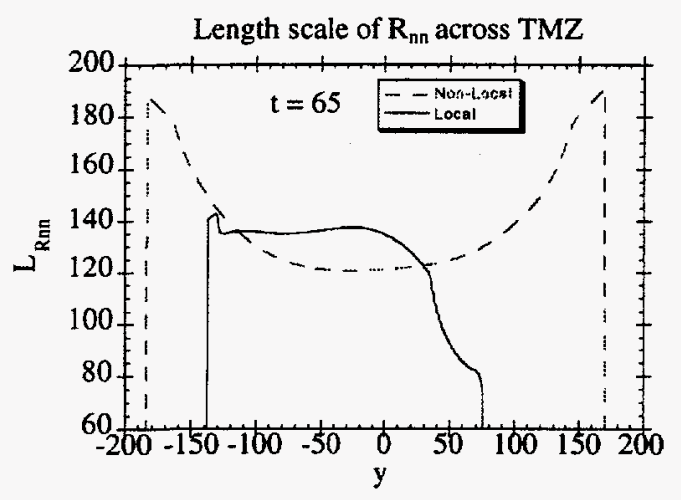

(c)

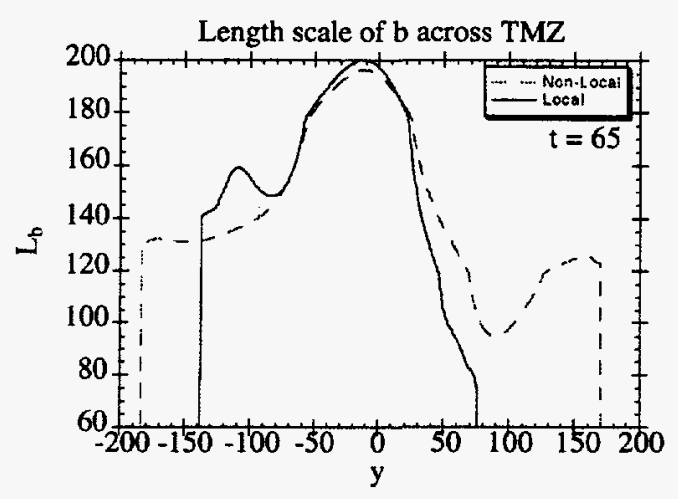

(b)

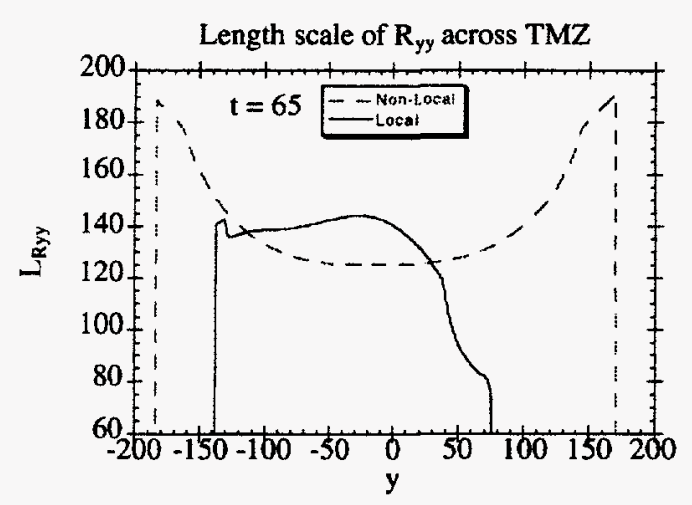

(d)

Fig. VII.D.5. Comparison of the profiles of the length scales across the TMZ for a local and nonlocal run at $t=65$ for: (a) the net mass-flux velocity, $a$, (b) the specific volume-density correlation, $b,(c)$ the contraction of the Reynolds stress tensor, $R_{n n}$, and, $(d)$ the $R_{y y}$ component of the Reynolds stress tensor.

The calculation with the nonlocal source is considerably closer to the most-likely experimental value, but it is apparent that more precise data would be useful as a basis for validation of any modified form for $\mathrm{Q}\left(\mathrm{y}^{\prime}, \mathrm{y}\right)$ in the expression for nonlocal creation.

A calculation was performed for a somewhat different departure from the base case, to examine the consequences of modifying the low-wave-number initialization of $b(k)$. In the initial-condition formula for $b(k)$ in the interface computational cell,

$$
b(k)=\frac{\gamma_{1} k^{n}}{1+\gamma_{2} k^{n+5 / 3}}
$$




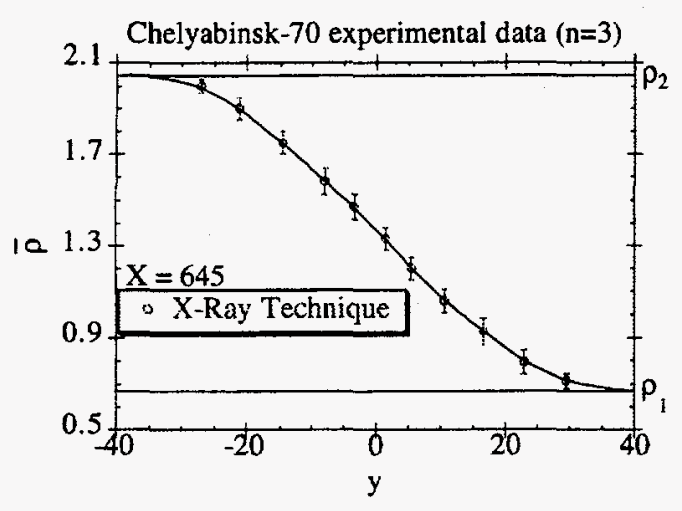

(a)

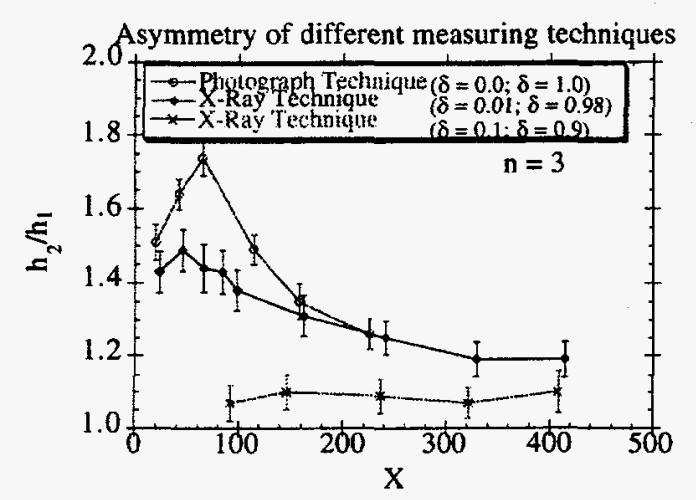

(c)

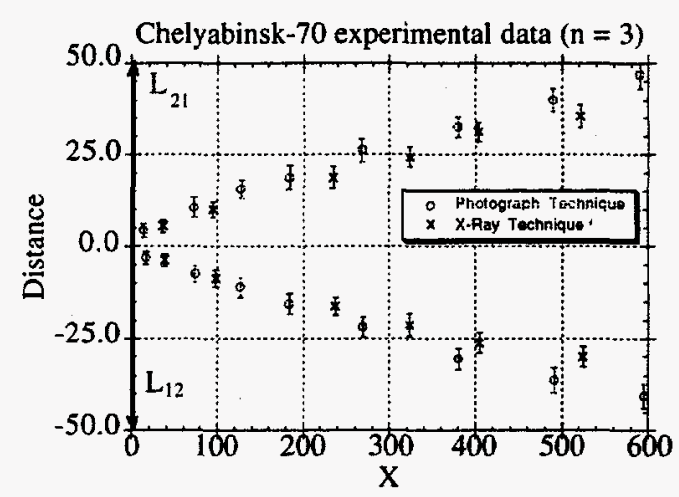

(b)

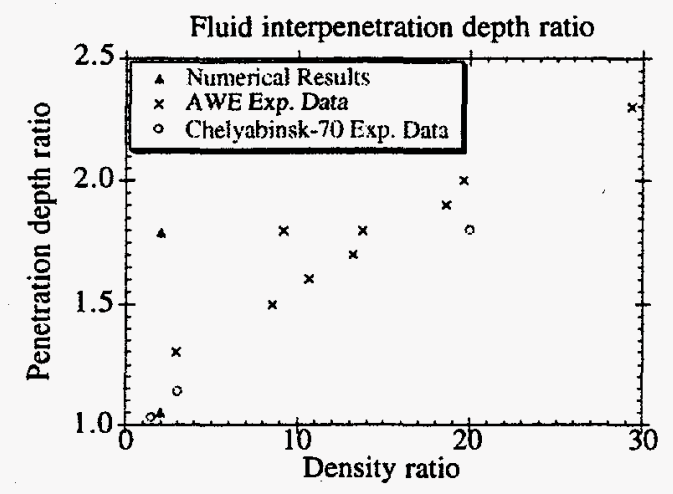

(d)

Fig. VII.D.6. Experimental data from Chelyabinsk-70 (Kucherenko et al. 1991) for: (a) concentration profile through TMZ, (b) bubble and spike penetration depth, (c) asymmetry of spike to bubble ratio, and (d) comparison of numerical results to experiments (Kucherenko et al. 1991; Smeeton and Youngs, 1987).

we used $n=4$ in the base case and $n=2$ in this modified run. Here $\gamma_{1}$ and $\gamma_{2}$ are the same as in the beginning of this section. All other conditions were the same for the two calculations. The purpose for the comparison is to explore the possibility of nonuniqueness for late-time selfsimilarity as induced by the persistence of structure at low wave numbers. This intriguing question has been discussed at length by previous investigators. The $n \geq 4$ it is thought that a single self-similar form function will emerge (Batchelor, 1986); for $n<4$ there is reason to believe that the form function will persistently depend on the value of $n$. In fully threedimensional $\mathbf{k}$-space the continued influence of low-wave-number structure on self-similarity gives a rich set of potential form functions. In the current comparison we see that with $\mathbf{n}=2$ the differences from the base-case results are so slight, largely due to choice of modeling, that we 


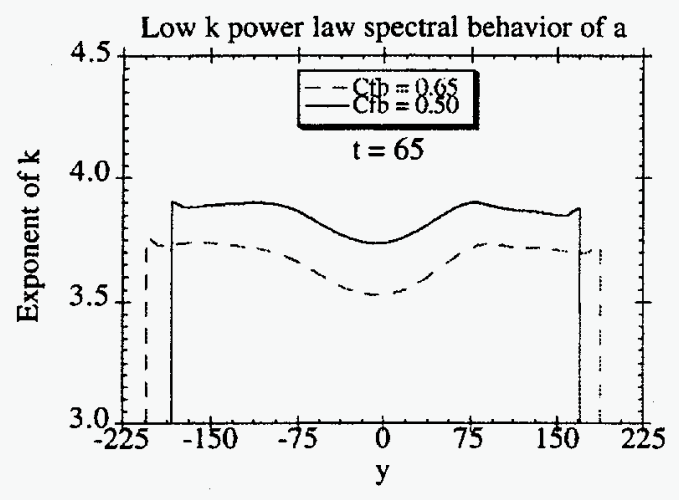

(a)

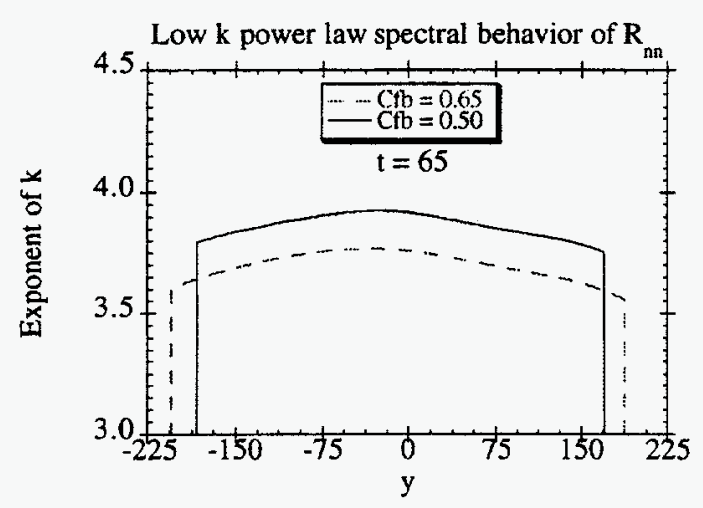

(c)

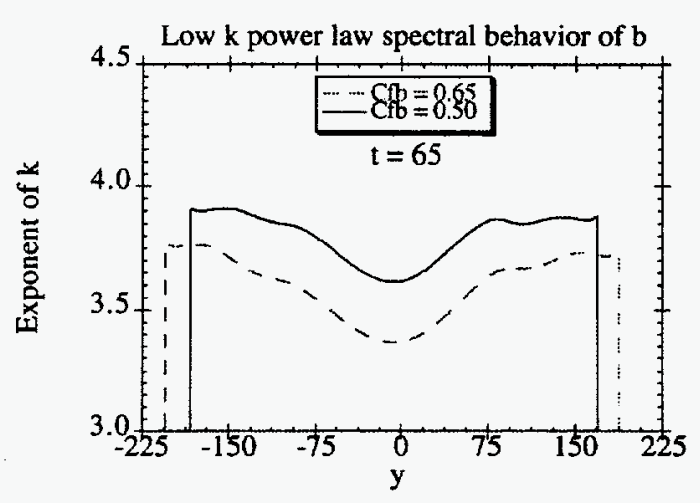

(b)

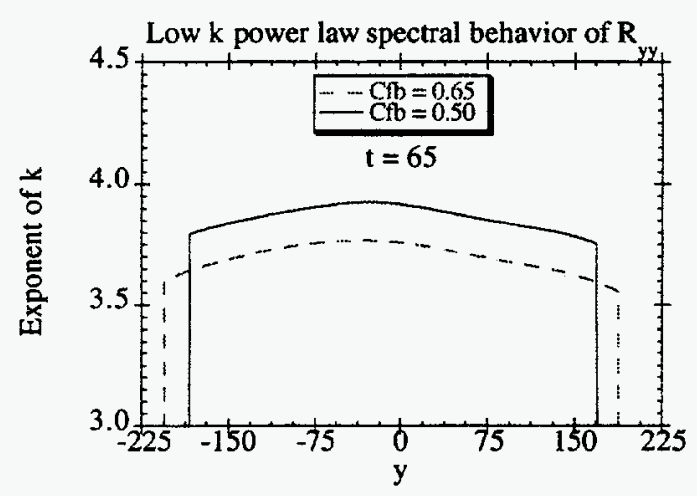

(d)

Fig. VII.D.7. Comparison of the low wave number power law behavior of $k$ at $t=65$ for two different values of $C f_{b}$ for: (a) the net mass-flux velocity, $a,(b)$ the specific volume-density correlation, $b,(c)$ the contraction of the Reynolds stress tensor, $R_{n n}$, and, $(d)$ the $R_{y y}$ component of the Reynolds stress tensor.

could not effectively illustrate them in figures. With $n=2$

- the values of $\mathrm{a}(\mathrm{k}), \mathrm{R}(\mathrm{k})$, and $\mathrm{R}_{\mathrm{yy}}(\mathrm{k})$ are slightly larger,

- $\mathrm{h}_{2} / \mathrm{h}_{1}$ is slightly smaller (asymptotically, $\mathrm{h}_{2} / \mathrm{h}_{1} \approx 1.03$ ),

- the TMZ spreads a bit faster, $\alpha=0.098$,

- the spectra all propagate slightly faster toward low wave numbers.

These trends are qualitatively consistent with the fact that $n=2$ gives larger $b(k)$ values for small wave numbers, persistently resulting in creation enhancement for $a(k)$ and in turn the Reynolds-stress components.

As a last calculational example, we discuss the effects that result from a change in $\mathrm{C}_{\mathrm{fb}}$, the "bubble-doubling" coefficient. In the base case, $\mathrm{C}_{\mathrm{fb}}=0.50$; in the comparison calculation $\mathrm{C}_{\mathrm{fb}}$ is increased to 0.65 . The most prominent of the results are shown in Fig. VII.D.7. As discussed in 
Section VIII, there are competing processes at low wave numbers, for which the three most prominent contributors are

- the initialization exponent, $n$, for $b(k)$,

- the value of $\mathrm{C}_{\mathrm{fb}}$,

- the nonlocal source for Reynolds stress.

The dominant effect is that of $\mathrm{n}$, which sets the exponent for all the variables to magnitudes not far from $\mathrm{n}$ and that persist into the self-similar stages. Nonlocality of source to Reynolds stress tends to increase the exponent; only the local source is spectral-form preserving (i.e., imparts the low-wave-numbers form of $\mathrm{a}(\mathrm{k})$ without distortion). The $\mathrm{C}_{\mathrm{fb}}$ contribution tends to decrease the exponent at low wave numbers for $b(k)$, with the effect spreading to $a(k)$ and thence to $R_{n n}(k)$ and $R_{y y}(k)$. The net effect of these influences is seen in Fig. VII.D.7, which shows the greatest change in $b(k)$, less in $a(k)$, and even less in the Reynolds-stress spectra. Also apparent in Fig. VII.D.7 is the greater width of the TMZ for the calculation with larger $C_{\mathrm{fb}}$. At high wave numbers the effects of this increase in $\mathrm{C}_{\mathrm{fb}}$ are negligible. Comparisons of self-similar spectral forms are not illustrated in the figures; the forms are almost precisely the same as for the base case; with $\mathrm{C}_{\mathrm{fb}}=0.65$ all of the normalized spectral forms show very slightly magnitudes at low wave numbers and slightly lower at high wave numbers. 


\section{SPECTRAL BEHAVIOR OF THE TERMS}

As compared to the case of decaying homogeneous constant density turbulence, the added complexity of inhomogeneity and variable density changes the spectral behavior of $R_{i j}(k)$. The additional variables that are a consequence of the variable density, namely $a_{i}(k)$ and $b(k)$, also contribute to altering the spectral shape of $R_{\mathrm{ij}}(\mathrm{k})$. In this section we examine the influence that these variables have on the spectra. In order to systematize the discussion, we first address the complexities associated with inhomogeneous turbulence and then discuss the resulting alterations in the spectra by splitting the spectra into two sections: (A) the high wave numbers and (B) the low wave numbers. For each section we identify the terms in the model that have the greatest influence on the behavior of the spectra. For each of the terms, we discuss how they interact with other terms in order to alter the spectrum.

Besnard et al. (1990) shows the existence of self-similarity upon substitution of the self-similar form for the homogeneous isotropic turbulence energy spectrum as suggested by Karman and Howarth (1938) into their spectral model. With our variable-density circumstances the relevance of these simple observations is even less apparent. In this section we show the basis for numerous deviations from simple self-similarity in these more general circumstances. In Section IX, we nevertheless describe a possible set of moment equations based on self-similarity. To proceed with this discussion, however, we will comment on the specific ways in which we can characterize the spectrum associated with the quantities of our model for variable density turbulence.

Here we state the general principal that we follow for this study based on our beliefs about the nature of the attainment and subsistence of the structure of spectral self-similarity: The entire evolution of spectral self-similarity is the direct result of a competition of processes that drive a flow. For the case of the decay of isotropic constant-density homogeneous turbulence, the identification of these competing processes is very simple and straightforward. The energy spectrum, $E(k)$, where $E(k)=E_{11}(k)+E_{22}(k)+E_{33}(k)$, is altered only by the transfer of energy among different scales. This transfer can be represented by the local diffusion model (Leith, 1967) which accounts for cascade to higher wave numbers as well as the diffusion to both higher and lower wave numbers. For decaying constant density isotropic turbulence, the transfer of energy through the inertial range of the spectrum is conservative. In Besnard et al. (1990) a discussion is given pertaining to the relationship between spectral self-similarity and the competition between the cascade and viscous dissipation terms.

For the case of constant density homogeneous anisotropic turbulence with a source, such as an infinite shear layer, the balance between processes must also include the source, which in this case is due to the presence of the $E_{12}(\mathrm{k})$ spectrum coupled to the mean flow gradient. The $E_{12}(\mathrm{k})$ spectrum acts as a source to the $E(k)$ spectrum at the smaller wave numbers. This is possible due 
to the fact that the $E_{12}(k)$ spectrum goes as $k^{-7 / 3}$ and the $E(k)$ spectrum goes as $k^{-5 / 3}$. Thus we have a source, namely $E_{12}(k)$, continuously pumping energy into the $E(k)$ spectrum at the lower wave numbers, the conservative cascade of energy to the higher wave numbers, and the final decay of energy at the highest wave numbers. The idea here is that, similar to the isotropic case, once an equilibrium is reached among the different processes, the energy spectrum assumes a self-similar time independent shape.

For the case of inhomogeneous anisotropic constant density turbulence, such as a free shear layer, additional facets such as the diffusion, advection, and nonlocal sources to energy in physical space significantly complicate this balance of processes. With these additional facets to the flow, the cascade of energy through the inertial range from low to high wave numbers is no longer a conservative process. Account must also be taken for the transfer of energy in physical space. For example, in a given control volume in $(\mathrm{k}, \mathrm{y})$-space, the transfers of the various Reynolds stress tensors are still conservative; but the added dimension in the $y$-direction distinguishes this from the homogeneous case. The sources are still predominately at the small wave numbers, and the sink is still at the highest wave numbers. For this case, the inertial range contains sinks and sources due to the inhomogeneity resulting in a nonconservative cascade of energy through the inertial range.

Another complexity that anisotropy adds is the addition of the remaining six components of the Reynolds stress tensor that do not contribute for the case of isotropic turbulence. This facet of the self-similarity is difficult to represent schematically. The coupling of these components with one another and the exchange of energy that occurs between them also complicate the competition of processes.

For flows that contain a time dependent source, the characteristic time for the different processes that are responsible for the transfer of energy becomes an issue. For example, if a flow has reached a state of equilibrium between all of its competing processes that transfer energy and the driver of the flow undergoes some type of transient, then the rate of return of the flow to a state of equilibrium is governed by a competition between these characteristic times. The processes must compete with one another until an equilibrium is reached and the flow is once again selfsimilar.

If we now allow the density to vary in the inhomogeneous circumstance, the competition between processes becomes even more involved due to the transfer of energy between the added functions that arise as a consequence of these density fluctuations. Referring to the equations of our model, we see that $b$ drives $a_{i}$ and $a_{i}$ in turn drives $R_{i j}$ which feeds back into $b$ and $a_{i}$. Of course, a transient in the driver of the flow will also result in greater complications.

As an example of the spectral altering effects of inhomogeneity, consider the turbulent viscosity, $v_{t}$, which is present in the spatial diffusion term of each equation of our model. Letting 
$\varphi$ represent any one of our transported turbulence variables, the turbulent viscosity appears in our model equation like

$$
\frac{\partial \varphi(\mathbf{k}, \mathbf{x}, \mathrm{t})}{\partial \mathrm{t}}=\frac{\partial}{\partial \mathrm{x}_{\mathrm{n}}} v_{\mathrm{t}}(\cdots) \frac{\partial \varphi(\mathbf{k}, \mathbf{x}, \mathrm{t})}{\partial \mathrm{x}_{\mathrm{n}}}+\ldots
$$

The turbulent viscosity can be modeled in either a local (in k-space) version, e.g.,

$$
v_{t}(x, k, t)=\frac{\sqrt{k E(x, k, t)}}{k}
$$

which maintains k-dependence; or any one of various types of nonlocal (in k-space) versions, e.g.,

and

$$
v_{t}(k, x, t)=\int_{0}^{k} \frac{\sqrt{k^{\prime} E\left(k^{\prime}, x, t\right)}}{\left(k^{\prime}\right)^{2}} d^{\prime}
$$

$$
v_{t}(x, t)=\int_{0}^{\infty} \frac{\sqrt{k E(x, k, t)}}{k^{2}} d k
$$

the first of which maintains k-dependence and the latter which is independent of $\mathrm{k}$. The second of the two nonlocal versions of $v_{t}$ is a consequence of considering the effect of all scales of the turbulence on the random walk process associated with diffusion. This consideration renders the turbulent viscosity independent of $k$. The first version accounts for only the wave numbers smaller than the point in the spectrum. The local version represents only the influence on the diffusive process due to eddies associated with the same wave number. A third version could be suggested that would account for the action at all wave numbers larger than the wave-number location, suggesting that it is the smaller turbulent structures that influence the diffusive process the most. This type of representation would also be dependent on $\mathrm{k}$.

The point to be emphasized here is the nature of the modeling for the turbulent viscosity, i.e., whether or not it is dependent on the wave number $k$ (roughly associated with the size of the eddies). For the case where $v_{t}$ is independent of wave number, the effective diffusion of the transported variable will be a flux proportional to the gradient that remains constant throughout the entire spectrum. For this particular case, as shown in Eq. (VIII.1) the diffusion term has no explicit dependence on $\mathrm{k}$ so that $\partial \varphi / \partial t$ has the same spectral form as $\varphi$ itself, resulting in no alterations of the spectrum due to the diffusion term. Since the same sink/source is "felt" on the entire spectrum, the diffusion term cannot be responsible for altering the neighboring (in physical space) spectra in any way. Contrarily, if a version of the turbulent viscosity is used which renders $v_{t}$ dependent on the wave number $\mathrm{k}$, the variation in the diffusion for a given point in physical $\mathrm{y}$ - 
space for different magnitudes of $\mathrm{k}$ will alter the spectral shape of $\varphi$. The variation in the level of diffusion in physical space, $y$, for all the different wave numbers effectively alters the neighboring spectra resulting in different spectral behaviors.

Thus, the two main effects due to the inhomogeneity are (1) lateral spectrum changes as we move in y-space, and (2) the "inertial range" is altered even at the same point in y-space. That is to say, that not only the adjacent spectra are altered, but also the cascade flux in k-space is no longer constant in the "inertial range."

To illustrate this quantitatively, suppose that the energy tensor, $E(k)$, behaves like $k^{-5 / 3}$ through the inertial range. Then the local version of the turbulent viscosity, Eq. (VIII.2), will behave as $\mathrm{k}^{-4 / 3}$ through the inertial range, and since the form of the diffusion terms in this model is that found in Eq. (VIII.1), a quantity that behaves like $\mathrm{k}^{-9 / 3}$ through the inertial rage will be diffused to the neighboring locations in physical space.

In view of the above behavior, one may ask the question: does this behavior mean that we have lost the type of self-similarity that has been observed for the case of decaying constant-density homogeneous isotropic turbulence (Besnard et al. (1990))? The answer to this question is probably not. We believe that the spectrum evolves to a self-similar shape. The important concept to recognize here is the method used to detect a self-similar evolution of the spectrum and the fact that $k_{\max }$ (the value of $k$ at which the spectrum attains an extremum) exhibits spatial dependence.

In the process of determining if a spectrum is evolving self-similarly, two things must be done to a time sequence of spectral plots: (1) the magnitudes of the spectra are rescaled such that the extremum of the spectra coincide, and (2) the positions of the spectra are rescaled in such a way that all spectra attain their extremum at the same wave number. With these two rescalings, the spectra are said to be evolving self-similarly if a time sequence of spectra can be made to overlay one another. The degree to which one may say that the spectrum is evolving self-similarly is the degree to which the overlaid plots coincide.

To rescale spectra such that the maxima coincide, the value of $k_{\max }$ must be known. A feature of this study is the recognition that $\mathrm{k}_{\max }$ is a function of the spatial variable $\mathrm{y}$. With this identification of a spatially dependent $k_{\max }$, we can effectively rescale the spectra so as to test for the self-similar behavior of the spectrum as functions of both $y$ and $t$. This merely amounts to a different rescaling for each position in y-space through the mixing layer.

The evolution of the spectrum may converge to an exact self-similar shape but we are discovering the importance of the concept of "almost" self-similar, and the evolution to a generic form of the spectra.

\section{A. High Wave Numbers}

We now examine an important characteristic of the $a_{i}(k)$ spectrum at high wave numbers. It is shown that as a consequence of the decay terms in the $a_{i}(k)$ equation, the $a_{i}(k)$ spectrum falls off 
much more rapidly for high wave numbers than do the spectra for either $b(k)$ or $R_{i j}(k)$. Since $a_{i}(k)$ is the principal source to both $b(k)$ and $R_{i j}(k)$, this behavior of the $a_{i}(k)$ spectrum results in a source term to $b(k)$ and $R_{i j}(k)$ that is effectively localized to the lower wave numbers. This behavior of the source term is closely aligned with the behavior of the source term due to a shear layer in a constant density flow as previously discussed in this section.

The behavior of the high wave-number part of the $\mathrm{a}_{\mathrm{i}}(\mathrm{k})$ spectrum is dominated by a competition among four terms in the transport equation of $\mathrm{a}_{\mathrm{i}}(\mathrm{k})$. These four terms are the two source terms, i.e., the $[b(k)] \partial \bar{p} / \partial x_{i}$ term and the $\left(R_{i n}(k) / \bar{\rho}^{2}\right)\left(\partial \bar{\rho} / \partial x_{n}\right)$ term, and the two drag terms, i.e., the $\left(-C_{r p 1} k^{2} \sqrt{a_{n}(k) a_{n}(k)}\right) a_{i}(k)$ term and the $\left(-C_{r p 2} k \sqrt{k R_{n n}(k) / \bar{\rho}}\right) a_{i}(k)$ term. A simple analysis of the competing terms in the transport of the $a_{i}(k)$ equation shows that the high wave-number behavior of $a_{i}(k)$ will either vary as $k^{-11 / 6}$ or $k^{-7 / 3}$, depending on which drag term is dominating. Due to the lack of any drag-like decay terms in the $R_{\mathrm{ij}}(\mathrm{k})$ and $\mathrm{b}(\mathrm{k})$ equations, the behavior of these quantities is dominated by the condition of nearly constant flux in $\mathrm{k}$ space, so that they vary closely as $\mathrm{k}^{-5 / 3}$ for the high wave numbers (see Fig. VII.B.3(b)). To demonstrate the consistency of these conclusions of $\mathrm{a}_{\mathrm{i}}(\mathrm{k})$ for high wave numbers, we use the fact that the two source terms in the $a_{i}(k)$ equation vary nearly as $k^{-5 / 3}$ and set up a balance among them and the decay terms. For this examination of the high wave numbers, let us represent the vector component $a_{i}$ as some constant multiplied by a power, $n$, of $k$, i.e.,

$$
\mathbf{a}_{\mathrm{i}} \rightarrow \mathrm{a}_{0} \mathbf{k}^{\mathrm{n}}
$$

Likewise, we can closely approximate $R_{\mathrm{ij}}$ and $b$ in a similar fashion:

and

$$
R_{i j} \rightarrow R_{0} k^{-5 / 3}
$$

$$
b \rightarrow b_{0} k^{-5 / 3} \text {. }
$$

With these expressions, a balance among the source and decay terms on the right side of the $a_{i}(k)$ equation becomes

$$
\left(b_{0} \frac{\partial \bar{p}}{\partial y}+\frac{R_{0}}{\bar{\rho}^{2}} \frac{\partial \bar{\rho}}{\partial y}\right) k^{-5 / 3} \approx\left(C_{r p l} k\left|a_{0} k^{n}\right|+C_{r p 2} k^{\frac{k R_{0} k^{-5 / 3}}{\bar{\rho}}}\right) a_{0} k^{n}
$$

From this expression, if the $\mathrm{C}_{\mathrm{rp} 1}$ term is dominating, the following balance must exist between the exponents of $\mathrm{k}$ : 


$$
\frac{-5}{3}=2(n+1)
$$

from which we see that we get $n=-11 / 6$. This is the expected power of the high wave-number behavior of $\mathrm{a}(\mathrm{k})$ if the $\mathrm{C}_{\mathrm{rp} 1}$ term dominates the decay.

If the $\mathrm{C}_{\mathrm{rp} 2}$ term dominates the high wave-number behavior then the following balance is established:

$$
-\frac{5}{3}=\frac{3}{2}-\frac{5}{6}+n
$$

from which we see that the power law behavior for the high wave number part of the spectrum for $a_{i}(k)$, becomes $n=-7 / 3$. Numerically we observe this (see Fig. VII.B.2(a)) and see that indeed it is the $\mathrm{C}_{\mathrm{rp} 2}$ term that dominates the decay process. (This dominance is also observed in calculations that show considerable insensitivity to the value of $\mathrm{C}_{\mathrm{rp} 1}$ through any reasonable variations of its magnitude.)

Now that we have established that for the self-similar regime, the high wave number for $\mathrm{a}_{\mathrm{i}}(\mathrm{k})$ goes as either $k^{-11 / 6}$ or $k^{-7 / 3}$ it follows that since $b(k)$ and $R_{i j}(k)$ both go as $k^{-5 / 3}$ for high wave numbers, $a_{i}(k)$ is a source that is localized to the low wave numbers for both $b(k)$ and $R_{i j}(k)$, and the assumption of cascade dominance for them in the inertial range is confirmed. In this manner, this situation is much like any anisotropic flow. Since the source to both the $b(k)$ and the $R_{i j}(k)$ equation is localized to the low wave numbers, there is a region of nearly constant flux through the inertial range for both $b(k)$ and $R_{i j}(k)$ allowing the spectrum to develop into a $k^{-5 / 3}$ spectral behavior. The flux is constant in this region for $b(k)$.

We should note however, that if $v_{\mathrm{t}}$ varies with $\mathrm{k}$, then there is a possible modification to the inertial spectra for $b(k)$ and $R_{i j}(k)$ arising from variations with $k$ of the $y$-direction diffusive flux (refer to earlier in this section).

Inversely, for high wave numbers, $b(k)$ creates $a_{i}(k)$ in a $k^{-5 / 3}$ fashion across the inertial range. However, as stated above, the drag terms of $a_{i}(k)$ dominate in this region, which drives the spectrum to a behavior more closely dominated by these drag terms.

\section{B. Low Wave Numbers}

We have seen the effective localization to small wave numbers of the source term to $R_{\mathrm{ij}}(\mathrm{k})$ and $b(k)$ due to the decay terms in the $a_{i}(k)$ equation. We now examine the low wave-number behavior of the sources to this model. We first examine the source term to the $R_{\mathrm{ij}}(\mathrm{k})$ equation and discuss the modifications resulting from the nonlocal extension to this term, which we have implemented into the model. We then describe the low wave number behavior of the source terms to the $a_{i}$ and 
b equation and other terms that play significant roles in the spectral modifications at low wave numbers.

The main source term to the $R_{\mathrm{ij}}(\mathrm{k})$ equation is composed of the net mass fluxing velocity, $\mathrm{a}_{\mathrm{i}}$, coupled to the mean pressure gradient, i.e., $\left[a_{i}(k)\right] \partial \bar{p} / \partial x_{j}+\left[a_{j}(k)\right] \partial \bar{p} / \partial x_{i}$. This term was examined in Section V. Past authors of single-point and two-point models have treated this source term due to the mean pressure gradient as a purely local term (Besnard et al., 1987; Besnard et al., 1990; Andronov et al., 1982). That is to say, the production of $R_{i j}$ at a point $\mathbf{x}$ is influenced by the mean pressure gradient only at that same position, $\mathbf{x}$. Due to incompressibility, we know that acoustic signals transfer the effects of the mean pressure changes instantaneously throughout the entire fluid, a global mechanism. In our model, in addition to the spectral formulation, we also formulate this source term to $R_{\mathrm{ij}}(\mathrm{k})$ nonlocally in physical space (see Section $\mathrm{V}$ ). The formulation of this nonlocal source term is easier to specify for a spectral model as opposed to a single-point model. The nonlocal spectral formulation is chosen to agree with the known spectral behavior for Rayleigh-Taylor analysis at low wave numbers. The additional information regarding length scales resulting from a spectral formulation enhances our ability to capture the global effects due to incompressibility.

However, as compared to a local source term for $R_{i j}(k)$, a nonlocal source term does change the spectral behavior of the model. The far reaching effects of the nonlocal term, i.e., the $\left|y^{\prime}-y\right|$ factor in the exponential of the $Q$ function as discussed in Section $V$, directly modify the $R_{i j}(k)$ spectrum and indirectly modify the $a_{i}(k)$ and $b(k)$ spectra through the coupling to $R_{n n}(k)$ in the local cascade rate and through the coupling to $R_{y y}(k)$ in the density gradient source term to $a_{i}(k)$.

Here we examine the modifications to the spectral behavior of the model due to the nonlocal source in $R_{i j}(k)$. We proceed by examining the two-dimensional $(y, k)$ shape of a purely local source to $R_{i j}(k)$ within the TMZ. Since $a_{i}(k)$ is zero outside of the TMZ (no net mass flux), the local source, $S_{i j}^{L}(y, k, t)$, to $R_{i j}(k)$ is likewise zero outside of the TMZ. We show the mechanism by which the local source undergoes a spreading over physical space due to the nonlocal modifications to the local source $S_{\mathrm{ij}}^{\mathrm{L}}(\mathrm{y}, \mathrm{k}, \mathrm{t})$ where

$$
S_{i j}^{L}(y, k, t)=a_{i}(y, k, t) \frac{\partial \bar{p}(y, t)}{\partial x_{j}}+a_{j}(y, k, t) \frac{\partial \bar{p}(y, t)}{\partial x_{i}} .
$$

Figure VIII.B.1 gives a qualitative idea of the nature of the effects of a local source term on $\partial R_{i j}(k) / \partial t$. The wave number $k_{1}$ is the wave number at which $a_{i}(k)$ attains its extremum. The TMZ is shown as the horizontal strip. The drawings across the horizontal TMZ represent, qualitatively, the relative magnitudes of the local source term in the $R_{i j}(k)$ equation. The purpose of these sketches is to depict the influence of the spectral shape of $a_{i}(k)$ on the source term to 


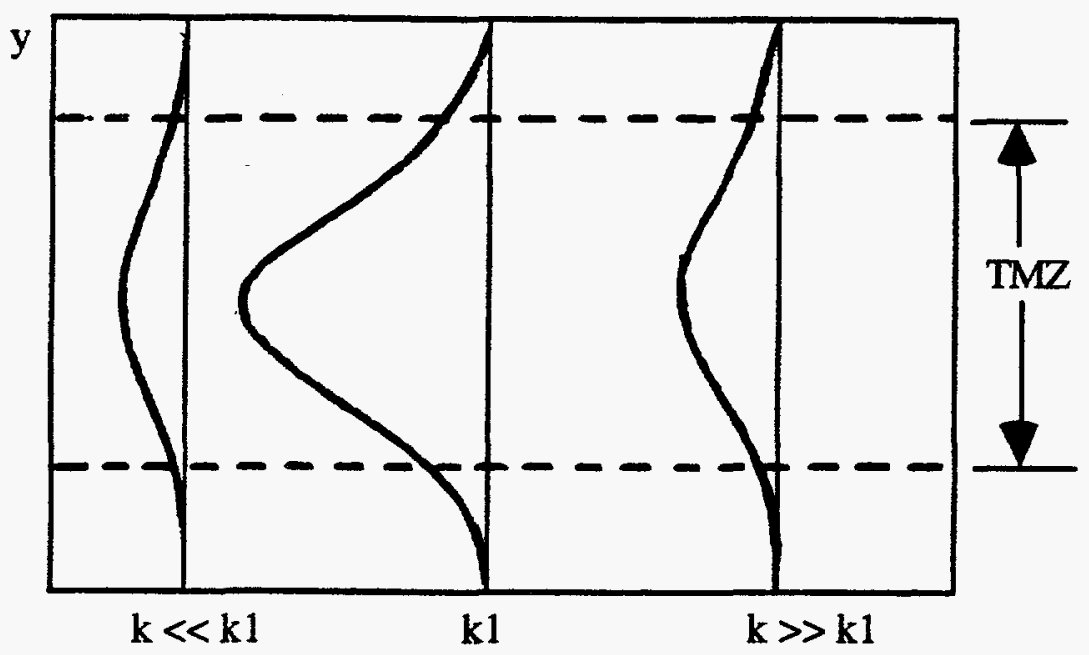

Fig. VIII.B.1. Schematic of the local source term across the TMZ as a function of $y$ and $k$. The maximum source corresponds to the wave number $k_{1}$ where the spectrum of $a(k)$ attains an extremum.

$\partial R_{i j}(k) / \partial t$. At $k=k_{1}$, the contribution to $\partial R_{i j}(k) / \partial t$ of the source term has a maximum while it tapers off to zero for both the low and high wave numbers of the spectrum. Notice also that the local source term goes to zero at both edges of the TMZ.

We now describe the smearing (across physical space) effect that the incorporation of the nonlocality has on the otherwise local source, $S_{i j}^{L}(y, k, t)$. As shown in Section $V$, the form of the nonlocal source term is derived from considerations of Rayleigh-Taylor theory. The nonlocal source is written as

$$
\int_{-\infty}^{+\infty} S_{i j}^{L}\left(y^{\prime}, k, t\right) Q\left(y^{\prime}, y, k, t\right) d y^{\prime} .
$$

We see that the source to $R_{i j}(k)$ at point $y$ receives contributions from $S_{i j}^{L}$, the local term, integrated over all space; and it is the $Q$ function that couples the global effects of $S_{i j}^{L}$ back to the point of production. Since the $Q$ function has the form

$$
Q\left(y^{\prime}, y, k, t\right)=Q_{0}(k, t) \exp \left(-k\left|y^{\prime}-y\right|\right)
$$

it will be responsible for altering the shape of the spectrum due to the $\mathrm{k}$ in the exponential. (The $\mathrm{Q}_{0}(\mathrm{k}, \mathrm{t})$ is a normalizing function which guarantees a global conservation of the source term over physical space). 
If we first examine only the form of $Q\left(y^{\prime}, y, k, t\right)$ uncoupled from the source term, we see that the $k$ in the exponential gives very different forms for large and small values of $k$.

Figure VIII.B.2 shows the different forms the $Q$ function will take for different values of $\mathbf{k}$. The structural feature that is important to notice in Fig. VIII.B.2 is the narrow base width for large values of $\mathrm{k}$ as compared to the spread out structures for the smaller values of $\mathrm{k}$. When coupled to $\mathrm{S}_{\mathrm{ij}}^{\mathrm{L}}$, this feature represents the ability of the large structures of the flow (associated with small $\mathrm{k}$ ) to reach out and influence remote parts of the flow while the influence due to the smaller structures of the flow (associated with high $\mathrm{k}$ ) remains highly localized.

Now we couple the local source term, $S_{i j}^{L}\left(y^{\prime}, k, t\right)$ to the reaching term, $Q\left(y^{\prime}, y, k, t\right)$ and show how the coupling results in a smeared out nonlocal source, $S_{i j}^{N L}(y, k, t)$, to $\partial R_{i j}(k) / \partial t$ that extends past the edges of the TMZ in physical space. Figure VIII.B.3 represents this coupling for a generic value of $k$.

As before, the smooth hump-like figure represents the local source (figure on top), and the new feature to notice is how this local source is smeared out past the edges of the TMZ (figures on bottom). The points at the various y positions outside of the TMZ are used to show the form of the Q-function. (Only a few points are included for clarity). The point to be made with Fig. VIII.B.3 is similar curves for $Q$ exist for all values of $y$ outside of the TMZ, which become more narrow at the base for the larger values of $k$ and widen at the base for smaller values of $k$. As the integral in the nonlocal source term is performed, contributions are made to $\partial R_{i j}(k) / \partial t$ at positions that lie outside of the TMZ due to the $Q$ function "reaching" into the TMZ.

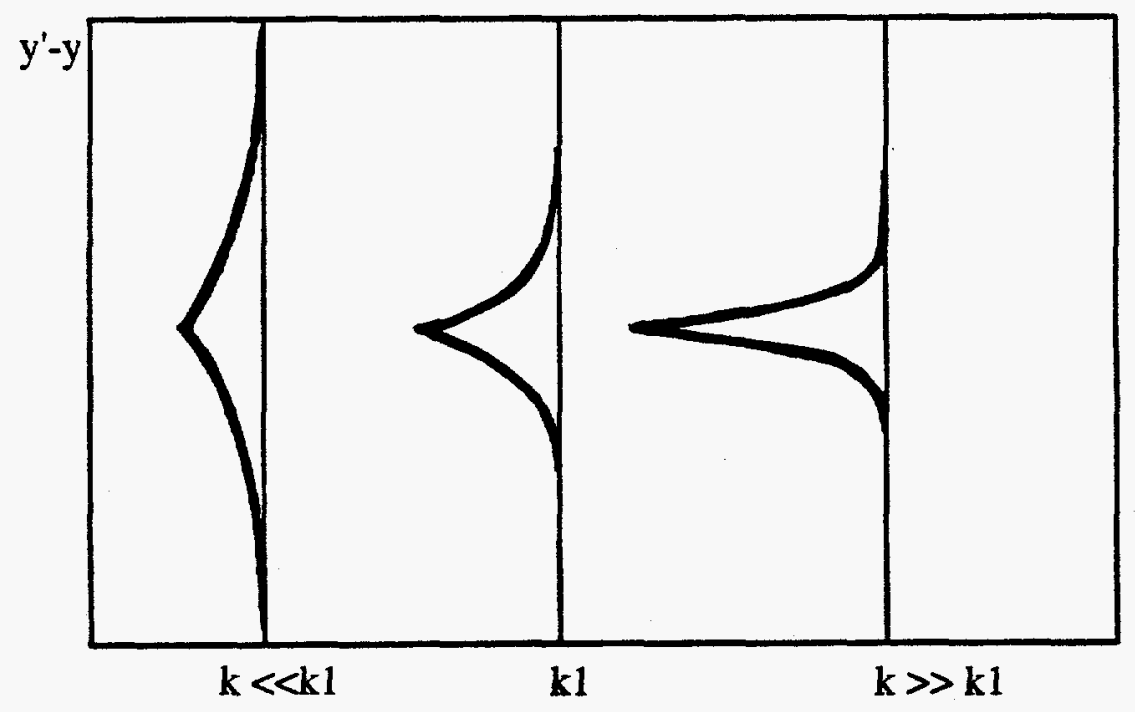

Fig. VIII.B.2. Schematic representation of the shape of the $Q$ function. Notice how the base in the y direction becomes more narrow for larger values of $k$. The area contained by each curve is the same due to normalization. 


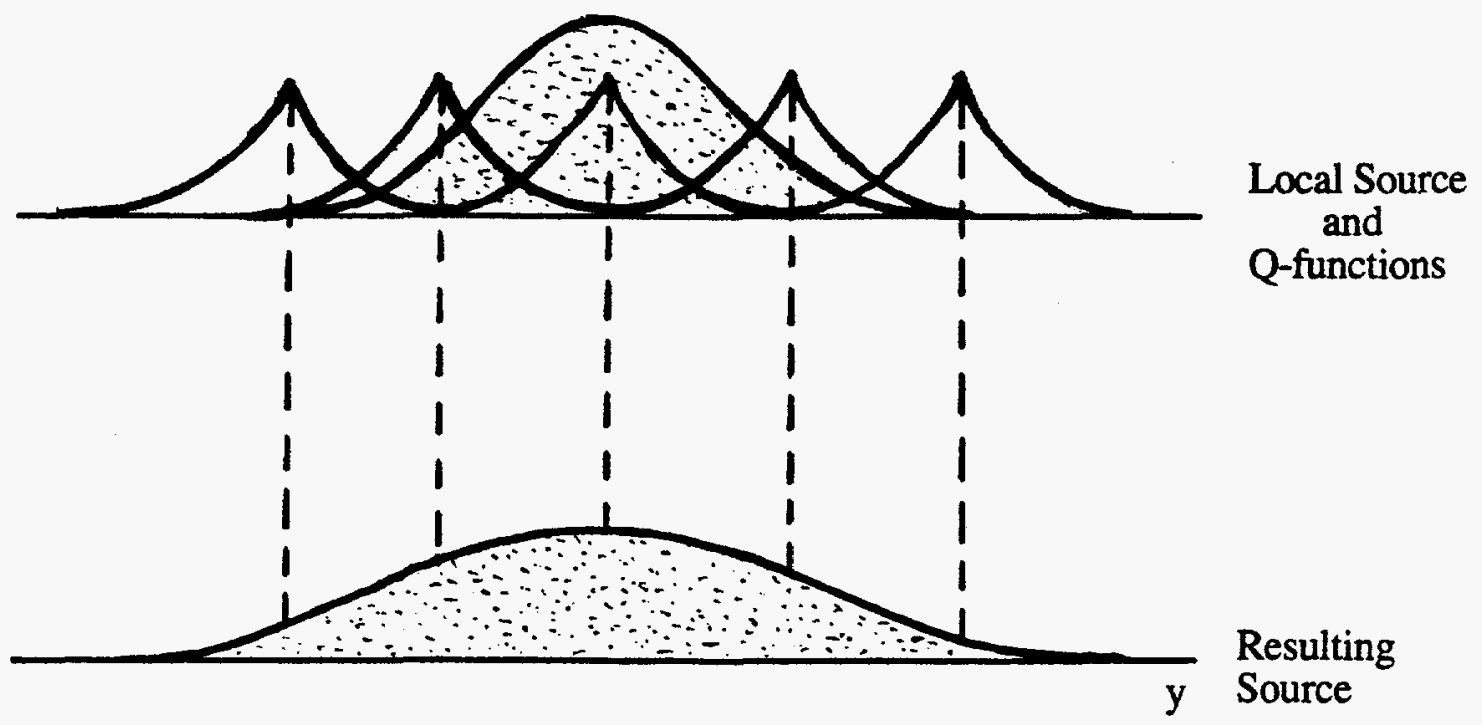

Fig. VIII.B.3. Schematic representation of the effect of the $Q$-function on the local source, $S_{\mathrm{ij}}^{\mathrm{L}}$, to $R_{i j}(k)$ for a generic wave numbers.

Figures VIII.B.2-VIII.B.3 show the effect of coupling the Q-function to the local source term to create a nonlocal source term. It is shown that as $k$ gets larger, the width of the base of the $Q$ function becomes more narrow hence retarding the ability of the nonlocal source term to reach out past the edges of the TMZ. In this limit, the Q-function spreading has little physical basis, and we require only that the result for large $k$ reduce to the local source. Recall that the extremum of the $a_{i}$ spectrum is at $k=k_{1}$. The consequence of this feature of the $a_{i}$ spectrum is that the effective local source to $\partial R_{i j} / \partial t$ will be larger for the wave numbers near $k_{1}$. The effect of the nonlocality is to flatten the peak of the source near the middle of the TMZ and to extend the source past the edges of the TMZ. The peak of the nonlocal source at the center of the TMZ will always be lower than the peak of the local source due to the normalization

$$
\int_{-\infty}^{+\infty} Q\left(y^{\prime}, y, k, t\right) d y^{\prime}=1
$$

For small wave numbers, the resulting nonlocal source is spread out over a relatively greater distance in physical space than for the larger wave numbers. This is to weight the nonlocal influence of the larger structures of the flow. Thus as we traverse the spectrum from $k=0$ to very large values of $k$; the $a_{i}$ spectrum starts at zero reaches a maximum and then asymptotes back to zero; the effect of the nonlocal $Q$ function on the source is to reach out to infinity for $k=0$ and asymptote to a local form for very large values of $k$. When coupled, these two effects produce a source that (1) is small in magnitude and extends well past the edges of the TMZ in physical space 
for small values of $\mathrm{k},(2)$ is large in magnitude and is more restricted in physical space for intermediate values of $k$, and (3) is small in magnitude and completely confined to the TMZ in physical space for asymptotically large values of $k$.

To summarize Figs. VIII.B.2-VIII.B.3, examine Fig. VIII.B.4 which compares the local and nonlocal shapes of the $\partial \mathrm{R}_{\mathrm{ij}} / \partial \mathrm{t}$ source term as a function of $\mathrm{y}$ for three different ranges of $\mathrm{k}$.

The three plots of Fig. VIII.B.4 show both the local and nonlocal source as a function of $y$ for different ranges of wave number. The main features of the plots are the decrease in the maximum of the source due to the nonlocality and the distance the nonlocal source extends past the local source as a function of wave number. These three plots of Fig. VIII.B. 4 can now be used to identify spectral modifications near the center and edge of the TMZ. A vertical strip is drawn through the edge of the TMZ as well as the middle of the TMZ $(y=0)$ to help identify the spectral tendency as to how the nonlocal source term modifies the spectral behavior of $\partial R_{\mathrm{ij}} / \partial \mathrm{t}$. The effect at the edge of the TMZ due to the nonlocality in the source term to $\partial \mathrm{R}_{\mathrm{ij}} / \partial \mathrm{t}$ is an increase in curvature for the small wave numbers, an increase in the absolute magnitude of the source as well as a migration of $\mathrm{k}_{\max }$ to lower wave numbers. The effect at the center of the TMZ due to the nonlocality in the source term to $\partial \mathrm{R}_{\mathrm{ij}} / \partial \mathrm{t}$ is a decrease in the curvature of the spectrum at the lower wave numbers, a lower absolute magnitude of the source at the centerline, and a migration of $k_{\max }$ to higher wave numbers. This is shown in Fig. VIII.B.5.

The information from Fig. VIII.B.5 can now be used to determine qualitatively the effects of a nonlocal source on the behavior of $k_{\max }$ through the TMZ. The local source produces a $k_{\max }$ that is concave upward, as a function of $y$, consistent with the largest turbulent length scales at the centerline of the TMZ, (Scale $=1 / \mathrm{kmax}$ ). The nonlocal source produces a $\mathrm{k}_{\max }$ curve through the TMZ that is concave downward, consistent with the larger turbulent length scales found at the edges of the TMZ. Inspection of Fig. VII.D.5(c) and (d) indeed verifies this behavior.

In the limit as $k \rightarrow 0$, the value of $n$ is preserved at the initial magnitude. From the nonlocal source term for example with $R_{n n}$ going as $k^{n} \exp [2 t \sqrt{k(A t) g}]$ for $k$ sufficiently small, this becomes, to leading order,

$$
\mathrm{k}^{\mathrm{n}}\{1+2 \mathrm{t} \sqrt{\mathrm{k}(\mathrm{At}) \mathrm{g}}+\ldots\}
$$

which shows the preservation of $\mathrm{k}^{\mathrm{n}}$ in the limits as $\mathrm{k} \rightarrow 0$ and also the emergence of a $\mathrm{k}^{\mathrm{n}+1 / 2}$ contribution in the spectra from near $k=0$. This effect can only be visible if $\mathrm{C}_{\mathrm{fb}}$ is sufficiently small indeed having a magnitude considerably less than that which is required for agreement with experiment. 


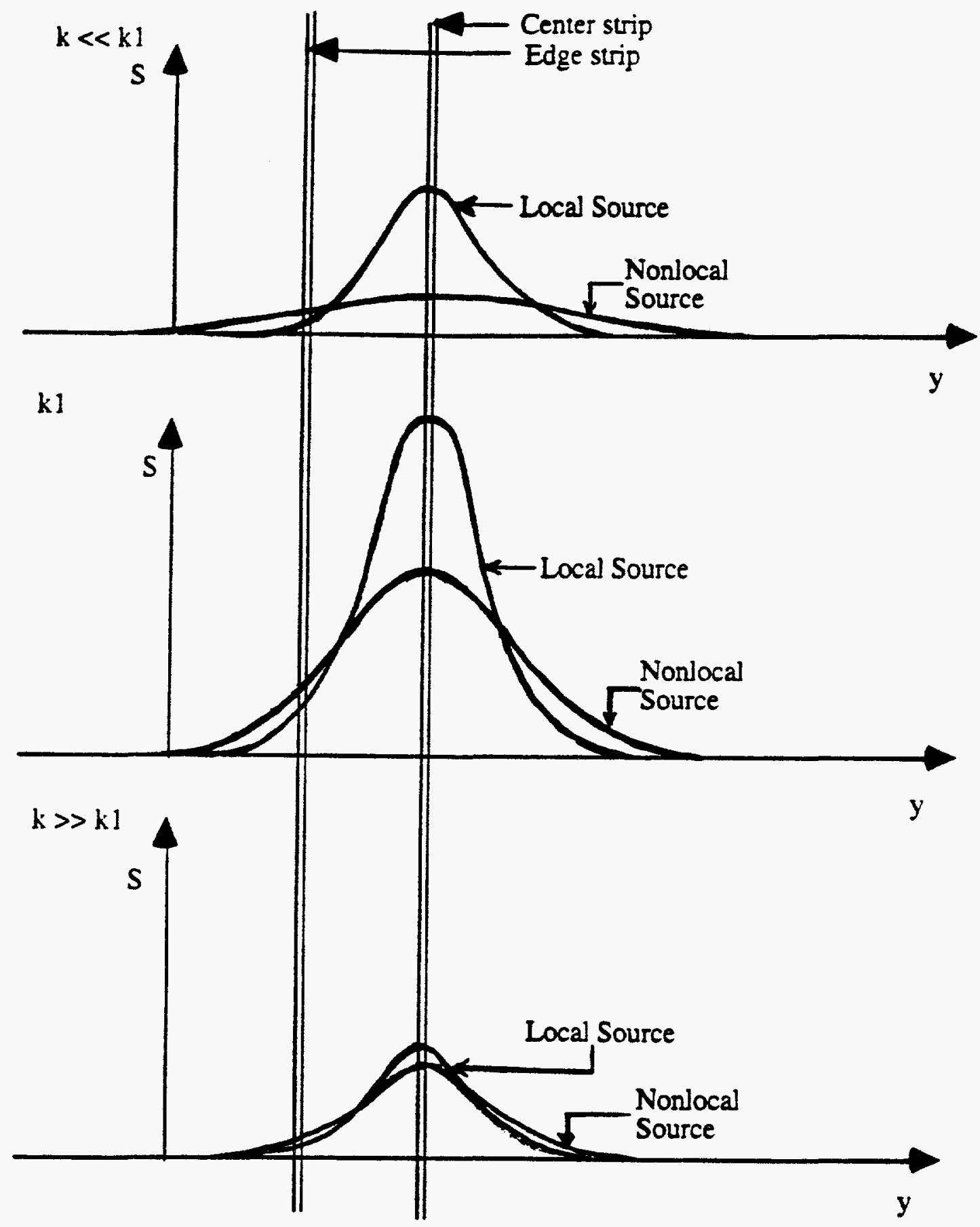

Fig. VIII.B.4. Schematic to represent the different structures through the TMZ of the nonlocal source term to $\partial \mathrm{R}_{\mathrm{ij}} / \partial \mathrm{t}$ due to the dependence of the nonlocality on wave number $k$. 


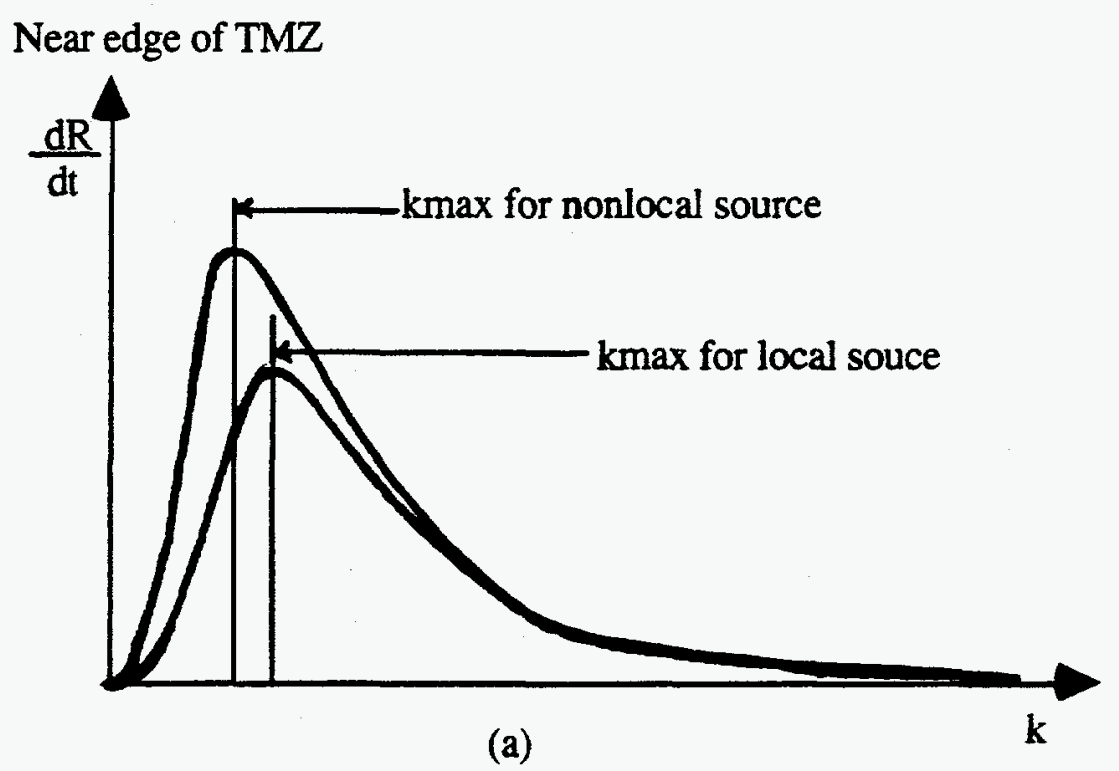

At center of TMZ

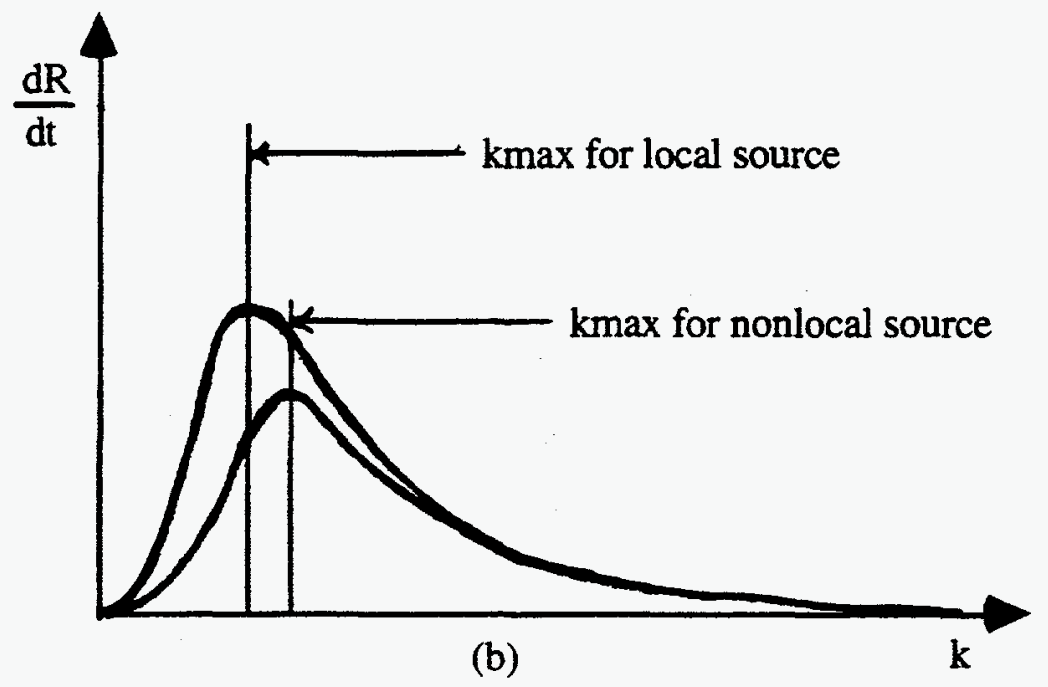

Fig. VIII.B.5. Schematic representation of the spectral modifications to the time rate of change of the Reynolds stress tensor due to a nonlocal source term for: (a) the edge of the TMZ and (b) the center of the TMZ.

In summary, the nonlocal source term to $R_{i j}$ affects the spectrum differently as we traverse the TMZ. Near the centerline of the TMZ the power, $n$, which dictates the power law behavior of the spectrum, $\mathbf{k}^{\mathrm{n}}$, is decreased for the low wave numbers and only slightly changed at the high wave numbers. Near the centerline, the $k_{\max }$ migrates towards higher wave numbers. As we move out towards the edge of the TMZ, the power $n$ is increased for the lower wave numbers, the higher wave numbers are only slightly affected, and $k_{\max }$ migrates to the lower wave numbers. The 
nonlocal source term to $\mathrm{R}_{\mathrm{ij}}$ has a major effect on the components of that tensor and are also felt indirectly on $a_{i}$ and $b$, principally through the alteration of cascade rates.

Two more items that alter the spectra for the low wave numbers are the initial conditions of $b$, i.e., the low wave number initialization for the $b$ spectrum and the $C_{\mathrm{fb}}$ term in the $b$ equation. The low wave-number behavior for the $\mathrm{b}$ spectrum and the $\mathrm{C}_{\mathrm{fb}}$ are in constant competition to determine the power law behavior for the low $\mathrm{k}$ parts of the spectra. Confined within the $\mathrm{b}$ spectrum this competition is most apparent. The $\mathrm{C}_{\mathrm{fb}}$ term is responsible for a wave-like propagation of the $\mathrm{b}$ spectrum from high to low wave numbers. This effect will increase the downward curvature of the b spectrum for the low wave numbers hence lowering the $n$ of the spectrum where $b \sim k^{n}$ for small $\mathbf{k}$. Hence it is apparent that this process will compete with the initial condition of the $b$ spectrum since the spectrum is continually migrating toward smaller wave numbers, thus allowing for a continual influence of the initial conditions. The effect of the $\mathrm{C}_{\mathrm{fb}}$ term however is to always lower the value of $n$ for the low wave number parts of the $b$ spectrum. The outcome of the competition of these two items then determines the low wave-number behavior for the $a_{i}$ spectrum that in turn influences the $R_{i j}$ spectrum.

One final class of terms to be mentioned that also alter the spectra at the low wave numbers are the $\mathrm{C}_{2}$ cascade terms in each of the equations (the $\mathrm{C}_{1}$ cascade terms are wave-like propagation to high wave numbers and have their principal manifestations at the high end of the spectrum). The $C_{2}$ cascade terms alter the spectral shape at a rate that depends on $R_{n n}(k)$. The alteration of this rate is thus affected by the nonlocal source, but this effect is considered to be secondary. The primary effect of the varying rate comes simply from the decrease in $\mathrm{R}_{\mathrm{nn}}$ with $\mathrm{y}$ for either type of source. 


\section{MOMENTS OF SPECTRAL EQUATIONS}

With the spectral transport equations, it is possible to solve a much wider scope of problems than can be addressed with single-point (nonspectral) formulations. Rapid variations in drive, for example, distort the nearly self-similar form functions into strongly circumstance-dependent expressions. Spectral equations work fine for describing such processes, but spectrally-integrated moment equations, based on assumed persistence of self-similarity, may exhibit large errors for such applications.

Spectral transport formulations are more complicated, however, as well as being much more expensive to use in numerical investigations with high-speed computers. Thus we use the two approaches in complementary fashion; in particular, we look at the spectral approach for clues to improving and extending the moment equations.

Steinkamp (1995) discusses the single-point turbulence transport equations in terms of previous nonspectral derivations, to which he has added some important clarifications and extensions. Significant difficulties are also described, especially in regard to size scales associated with both the turbulence and the interfacial configuration of mixing fluids. Decay of turbulence, for example, is directly related to the transfer rate from low to high wave numbers. Sources to turbulence can be strong in the "active" scales and weak in the "passive" scales, the latter simply balancing the buoyancy forces with drag. The resolution of these and other questions leads directly to the spectral formulations; the inverse process takes the spectral equations back to singlepoint form but augmented with representations of the principal spectral improvements that were previously missing.

Before proceeding with the derivations, we describe an example of the new single-point augmentation. For the simple self-similar mixing layer that dominates the considerations of this report, it is arguable that single-point transport equations are not required for both $a_{i}$ and $R_{i j}$. They are, after all, scaled in dimensionality by the same quantity, $\mathrm{g}$; and the spectral functions on which they are based are of universal form, so that knowledge of one implies that the other is known. Moreover, the scales associated with $b(k), a_{i}(k)$, and all the components of $R_{i j}(k)$ must be directly proportional to each other with universal ratios. Thus a pair of single-point equations for $a_{i}$ and $L_{a}$ should be sufficient to determine the entire process. These ideas are amply verified in the examples shown in Section VII.

Those examples show, however, that the moment self-similarity is destroyed (e.g., through reversal of $g$ ), the relationship among $a_{i}$ and the components of $R_{i j}$ are at once lost. Each evolves in its own particular response to the new circumstances, returning to a unique, universal relationship to each other only when (and if) self-similarity is again established (which is not the case during the de-mixing process), in which the width of the layer at the instant of $g$ reversal is 
firmly implanted as an additional dimensional quantity. In addition, the length scales associated with each of the spectra are now no longer universally proportional to each other. If the driver continues to vary in capricious fashion, these independent behaviors continue; and the discernment of relationships among variables requires the simultaneous transport analysis of them all.

The spectral equations describe all these "independent" but interactive behaviors. Can singlepoint (spectral-moment) equations do likewise? There is evidence to suggest that they can do fairly well in this regard. Simple $\mathrm{R}_{\mathrm{ij}}-\varepsilon$ models describe at least some types of transient circumstances, despite their derivation being equivalent to moments of the spectral form functions.

Likewise the BHR model and related models for variable density (multifluid) circumstances are surprisingly relevant to transient flows, even with compressibility.

At least two things must contribute to the success of single-point (nonspectral) formulations. One of these is the tendency for spectra to vary principally in magnitude during many kinds of transients, with little change in form (for example, a during g reversal in Section VII). The second is the tendency for rapid return to self-similarity after a transient change in drive. This latter, indeed, furnishes a significant criterion for spectral-moment transport validity in terms of the competition between change in rate of drive and return rate to self-similarity. With the spectral equations, this competition can be examined in detail.

In this section we first show the spectral moment derivations for the simplest TMZ, in which all ensemble averaged (layer-averaged) quantities are functions of $y, k$, and $t$ only. We write

$$
\begin{aligned}
a_{i}(y, k, t) & =a_{i}(y, t) L_{a}(y, t) F_{a}\left(k L_{a}(y, t)\right) \\
b(y, k, t) & =b(y, t) L_{b}(y, t) F_{b}\left(k L_{b}(y, t)\right) \\
R_{i j}(y, k, t) & =2 \bar{p}(y, t) K(y, t) L_{R}(y, t) F_{i j}\left(k L_{R}(y, t)\right) .
\end{aligned}
$$

Here, $K(y, t)$ denotes the turbulent kinetic energy and as usual, $\bar{\rho}(y, t)$ is the average density. The wave-number, $\mathrm{k}$, occurs only in the self-similar form functions, the Fs. The other quantities, $a_{i}, b, K, L_{a}, L_{b}$, and $L_{R}$, are all functions of $y$ and $t$; our goal in this section is to derive spectral moment equations to describe their transport.

The spectral moments are of an infinity of possible kinds. One of these is the weighting function, $\mathrm{k}^{\mathrm{o}}(\equiv 1.0)$. Another is $\mathrm{k}^{\mathrm{m}}$, for which Besnard et al. (1990) suggest a value of the exponent, $\mathrm{m}$, that is close to -1.0 . A third possibility is the spectral-splitting moment, $\mathrm{H}\left(\mathrm{k}_{1}-\mathrm{k}\right)$ in which this Heaviside function is 1.0 for $k<k_{1}$ and 0.0 for $k>k_{1}$. The value of each $k_{1}$ might lie at the maximum of the spectrum, or it might lie somewhere in the inertial range. We have not pursued this approach, although it has potential merit, for example, in identifying the active and passive parts of the $b$ spectrum. 
The spectral-moment transport equations that we require are six in number, two for each of the spectral equations for $a_{i}, b$, and $R_{n n}$, since each has two unknown functions of position and time, a magnitude, and a length scale. (At this stage we assume that the difference between $R_{n n}$ and $R_{y y}$ is sufficiently represented by their two different forms, $F_{n n}$ and $F_{y y}$.)

We substitute the self-similar forms of Eq. (IX.1) into the spectral transport equations of the model and integrate over $d k$ to get evolution equations for $\mathrm{K}(\mathrm{y}, \mathrm{t}), \mathrm{a}_{\mathrm{i}}(\mathrm{y}, \mathrm{t})$, and $\mathrm{b}(\mathrm{y}, \mathrm{t})$. The resulting transport equations depend on time and the physical-space variable, $y$, only. Due to the self-similar forms of Eq. (IX.1) length scales for each of the variables are incorporated into the new single-point transport equations. These length scales, namely $L_{a}, L_{b}$, and $L_{R}$, may be considered the inverse of the dominant wave number $\mathrm{k}_{\max }$ of the spectrum for each of the variables. In addition to performing the integrations just described, we also integrate the spectral transport equations over $\mathrm{k}^{\mathrm{m}} \mathrm{dk}$ for $\mathrm{m}=-1$ to get transport equations relating the single-point quantities to their associated length scales.

The functions are normalized to

$$
\int_{0}^{\infty} F_{\mathrm{a}}(\xi) \mathrm{d} \xi=\int_{0}^{\infty} \mathrm{F}_{\mathrm{b}}(\xi) \mathrm{d} \xi=\int_{0}^{\infty} F_{\mathrm{nn}}(\xi) \mathrm{d} \xi=1 .
$$

The maximum value of all three form functions is unity corresponding to the position $\xi=1$.

The process of substituting the self-similar forms from Eq. (IX.1) into the spectral transport equations and performing the integrations discussed above is straightforward. The local cascade terms are the only terms that warrant closer examination. The integration will be shown for the cascade of a generic variable, $\varphi$.

The generic cascade terms are expressed as

$$
\frac{\partial}{\partial \mathrm{k}}\left[\mathrm{k}^{2} \sqrt{\frac{\mathrm{kR}}{\bar{\rho}}}\left(-\mathrm{C}_{1} \varphi+\mathrm{C}_{2} \mathrm{k} \frac{\partial \varphi}{\partial \mathrm{k}}\right)\right] \text {, }
$$

Taking the m-th moment of this cascade term

$$
\int_{0}^{\infty} \mathrm{k}^{\mathrm{m}} \frac{\partial \mathrm{G}(\mathrm{k})}{\partial \mathrm{k}} \mathrm{dk}
$$


where

$$
G(k)=k^{2} \sqrt{\frac{k R_{n n}}{\bar{\rho}}}\left(-C_{1} \varphi+C_{2} k \frac{\partial \varphi}{\partial k}\right)
$$

Upon integrating by parts,

$$
\int_{0}^{\infty} k^{m} \frac{\partial G(k)}{\partial k} d k=\left.\left(k^{\prime}\right)^{m} G\left(k^{\prime}\right)\right|_{k^{\prime} \rightarrow \infty}-m \int_{0}^{\infty} k^{m-1} G(k) d k
$$

For this model, a good approximation for the high wave number behavior of the three quantities $a_{i}$, $b$, and $R_{n n}$ is that all three behave as $k^{n}$, where $n$ is at most $-5 / 3$. $G(k)$ remains constant for large values only when one samples the spectrum far enough to the right [away from the influence of $\left.a_{i}(k)\right]$. Upon examination of $G(k)$, it can then be seen that $G(k)$ approaches a constant value as $\mathrm{k} \rightarrow \infty$; thus for all values of $\mathrm{m}$ such that $\mathrm{m} \leq 0$, only the $\mathrm{m}=0$ moment will result in a nonzero contribution from the first term on the right side of Eq. (IX.6). Therefore the moment integral of the cascade terms can be expressed as

$$
\int_{0}^{\infty} k^{m} \frac{\partial G(k)}{\partial k} d k= \begin{cases}-m \int^{\infty} k^{m-1} G(k) d k & \text { for } m \neq 0 \\ \left.k^{2} \sqrt{\frac{k R_{n n}}{\bar{\rho}}}\left(-C_{1} \varphi+C_{2} k \frac{\partial \varphi}{\partial k}\right)\right|_{k \rightarrow \infty} & ; \text { for } m=0 .\end{cases}
$$

The term resulting from the moments for $m \neq 0$ on the right side of Eq. (IX.7) is a constant that represents the decay of the quantity $\varphi$ due the cascade action of the turbulence to higher and higher wave numbers.

With the discussion of the cascade terms completed, the self-similar forms from Eq. (IX.1) are now substituted into the spectral model equations and the $m=0$ and the $m=-1$ moments are taken. The resulting 3 transport equations for $a(y, t), b(y, t)$, and $K(y, t)$ are

$$
\begin{aligned}
\frac{\partial \bar{\rho} \mathrm{a}}{\partial \mathrm{t}}+\frac{\partial \bar{\rho} \mathrm{au}}{\partial \mathrm{y}}= & b \frac{\partial \overline{\mathrm{p}}}{\partial \mathrm{y}}-\left[\mathrm{N}_{4} \sqrt{\mathrm{aa}}+\mathrm{I}_{2} \sqrt{2 \mathrm{~K}\left(\frac{\mathrm{L}_{\mathrm{R}}}{\mathrm{L}_{\mathrm{a}}}\right)}\right] \frac{\bar{\rho} \mathrm{a}}{\mathrm{L}_{\mathrm{a}}} \\
& -\mathrm{N}_{3} \mathrm{~K} \frac{\partial \bar{\rho}}{\partial \mathrm{y}}+\mathrm{C}_{\mathrm{D}} \frac{\partial}{\partial \mathrm{y}} \bar{\rho} v_{\mathrm{t}} \frac{\partial \mathrm{a}}{\partial \mathrm{y}}
\end{aligned}
$$




$$
\begin{gathered}
\frac{\partial b}{\partial t}=\left(\frac{2 \bar{\rho}-\rho_{1}-\rho_{2}}{\rho_{1} \rho_{2}}\right) \frac{\partial \bar{\rho} a}{\partial y}+I_{1} \frac{b}{L_{b}} \sqrt{2 K\left(\frac{L_{R}}{L_{b}}\right)}+C_{D} \frac{\partial}{\partial y} v_{t} \frac{\partial b}{\partial y} \\
\frac{\partial \bar{\rho} K}{\partial t}+\frac{\partial \bar{\rho} K \tilde{u}}{\partial y}=\int_{-\infty}^{+\infty} a\left(y^{\prime}\right) \frac{\partial \bar{p}\left(y^{\prime}\right)}{\partial y} S_{0}\left(y, y^{\prime}\right) d y^{\prime}-N_{3} \bar{\rho} K \frac{\partial \tilde{u}}{\partial y} \\
+C_{D} \frac{\partial}{\partial y} v_{t} \frac{\partial \bar{\rho} K}{\partial y}-N_{2} \bar{\rho} \varepsilon
\end{gathered}
$$

and the corresponding three transport equations for their respective length scales are

$$
\begin{gathered}
\frac{\partial \bar{\rho} L_{a}}{\partial t}+\frac{\partial \bar{\rho} L_{a} \tilde{u}}{\partial y}=\left[\left(\frac{N_{7}}{N_{8}}\right)\left(\frac{L_{b}}{L_{a}}\right)-1\right] \frac{b L_{a}}{a} \frac{\partial \bar{p}}{\partial y}-N_{5} \bar{\rho} \sqrt{a a} \\
-I_{4} \bar{\rho} \sqrt{2 K\left(\frac{L_{R}}{L_{a}}\right)}+\left[N_{3}-N_{6}\left(\frac{L_{R}}{L_{a}}\right)\right] \frac{K L_{a}}{a} \frac{\partial \bar{\rho}}{\partial y} \\
+C_{D} \frac{\partial}{\partial y} \bar{\rho} v_{t} \frac{\partial L_{a}}{\partial y}+\frac{2 C_{D} \bar{\rho} v_{t}}{a}\left(\frac{\partial a}{\partial y}\right)\left(\frac{\partial L_{a}}{\partial y}\right) \\
\frac{\partial L_{b}}{\partial t}=\left(\frac{2 \bar{\rho}-\rho_{1}-\rho_{2}}{b \rho_{1} \rho_{2}}\right)\left[\frac{N_{8}}{N_{7}} \frac{\partial \bar{\rho} a L_{a}}{\partial y}-L_{b} \frac{\partial \bar{\rho} a}{\partial y}\right]+C_{D} \frac{\partial}{\partial y} v_{t} \frac{\partial L_{b}}{\partial y} \\
-\frac{N_{8}}{N_{7}} C_{f b}\left(\frac{\bar{v}^{2} a L_{a}}{b}\right) \frac{\partial}{\partial y}\left(\frac{\bar{\rho}}{\bar{v}}\right)+I_{3} \sqrt{2 K}\left(\frac{L_{R}}{L_{b}}\right)+\frac{2 C_{D} v_{t}}{b}\left(\frac{\partial L_{b}}{\partial y}\right)\left(\frac{\partial b}{\partial y}\right) \\
\frac{\partial \bar{\rho} L_{R}}{\partial t}+\frac{\partial \bar{\rho} L_{R} \tilde{u}}{\partial y}=\frac{1}{K} \int_{-\infty}^{+\infty} a\left(y^{\prime}\right) \frac{\partial \bar{\rho}\left(y^{\prime}\right)}{\partial y} S\left(y, y^{\prime}\right) d y^{\prime}-N_{9} \bar{\rho} L_{R} \frac{\partial \tilde{u}}{\partial y} \\
+\bar{\rho} C_{D} \frac{\partial}{\partial y} v_{t} \frac{\partial L_{R}}{\partial y}+\frac{2 C_{D} v_{t}}{K}\left(\frac{\partial \bar{\rho} K}{\partial y}\right)\left(\frac{\partial L_{R}}{\partial y}\right) \\
+N_{10} \bar{\rho} \sqrt{K},
\end{gathered}
$$


where

$$
\begin{aligned}
& \mathrm{N}_{1}=\int_{0}^{\infty}\left(\frac{1}{\xi}\right) \mathrm{F}_{\mathrm{nn}}(\xi) \mathrm{d} \xi \\
& N_{2}=\left[\xi^{2} \sqrt{2 \xi F_{n n}(\xi)}\left(C_{1} F_{n n}(\xi)-C_{2} \xi F_{n n}^{\prime}(\xi)\right)\right]_{\xi \rightarrow \infty} \\
& \mathrm{N}_{3}=2 \int_{0}^{\infty} \mathrm{F}_{\mathrm{yy}}(\xi) \mathrm{d} \xi \\
& \mathrm{N}_{4}=\mathrm{C}_{\mathrm{rp} 1} \int_{0}^{\infty} \xi^{2} \mathrm{~F}_{\mathrm{a}}^{2}(\xi) \mathrm{d} \xi \\
& \mathrm{N}_{5}=\left(\frac{\mathrm{C}_{\mathrm{rpl}}}{\mathrm{N}_{8}}\right)_{0}^{\infty} \xi \mathrm{F}_{\mathrm{a}}^{2}(\xi) \mathrm{d} \xi-\mathrm{N}_{4} \\
& \mathrm{~N}_{6}=\left(\frac{2}{\mathrm{~N}_{8}}\right)_{0}^{\infty} \int_{0}^{(}\left(\frac{1}{\xi}\right) \mathrm{F}_{\mathrm{yy}}(\xi) \mathrm{d} \xi \\
& \mathrm{N}_{7}=\int_{0}^{\infty}\left(\frac{1}{\xi}\right) \mathrm{F}_{\mathrm{b}}(\xi) \mathrm{d} \xi \\
& \mathrm{N}_{8}=\int_{0}^{\infty}\left(\frac{1}{\xi}\right) \mathrm{F}_{\mathrm{a}}(\xi) \mathrm{d} \xi \\
& N_{9}=\left(\frac{2}{N_{1}}\right) \int_{0}^{\infty}\left(\frac{1}{\xi}\right) F_{y y}(\xi) d \xi-N_{3} \\
& \mathrm{~N}_{10}=-\mathrm{N}_{2}+\left(\frac{1}{\mathrm{~N}_{1}}\right)_{0}^{\infty}\left[\sqrt{2 \xi \mathrm{F}_{\mathrm{nn}}(\xi)}\left(-\mathrm{C}_{1} \mathrm{~F}_{\mathrm{nn}}(\xi)+\mathrm{C}_{2} \xi \mathrm{F}_{\mathrm{nn}}^{\prime}(\xi)\right)\right] \mathrm{d} \xi \\
& +\left(\frac{1}{N_{1}}\right) \xi \sqrt{2 \rho F_{n n}(\xi)}\left[-C_{1} F_{n n}(\xi)+C_{2} \xi F_{n n}^{\prime}(\xi)\right]_{\xi \rightarrow \infty} \\
& \mathrm{I}_{1}=\left[\xi^{2} \sqrt{\xi \mathrm{F}_{\mathrm{nn}}\left[\xi\left(\frac{\mathrm{L}_{\mathrm{R}}}{\mathrm{L}_{\mathrm{b}}}\right)\right]}\left(-\mathrm{C}_{1} \mathrm{~F}_{\mathrm{b}}(\xi)+\mathrm{C}_{2} \xi \mathrm{F}_{\mathrm{b}}^{\prime}(\xi)\right)\right]_{\xi \rightarrow \infty}
\end{aligned}
$$




$$
\begin{aligned}
& \mathrm{I}_{2}=\mathrm{C}_{\mathrm{rp} 2} \int_{0}^{\infty} \xi \mathrm{F}_{\mathrm{a}}(\xi) \sqrt{\xi \mathrm{F}_{\mathrm{nn}}\left[\xi\left(\frac{\mathrm{L}_{\mathrm{R}}}{\mathrm{L}_{\mathrm{a}}}\right)\right]} \mathrm{d} \xi \\
& \mathrm{I}_{3}=\left(\frac{1}{\mathrm{~N}_{7}}\right)_{0}^{\infty}\left[\sqrt{\xi \mathrm{F}_{\mathrm{nn}}\left[\xi\left(\frac{\mathrm{L}_{\mathrm{R}}}{\mathrm{L}_{\mathrm{b}}}\right)\right]}\left(-\mathrm{C}_{1} \mathrm{~F}_{\mathrm{b}}(\xi)+\mathrm{C}_{2} \xi \mathrm{F}_{\mathrm{b}}^{\prime}(\xi)\right)\right] \mathrm{d} \xi-\mathrm{I}_{1} \\
& I_{4}=\left(\frac{C_{r p 2}}{N_{8}} \int_{0}^{\infty} F_{a}(\xi) \sqrt{\xi F_{n n}\left[\xi\left(\frac{L_{R}}{L_{a}}\right)\right]} d \xi-I_{2}\right. \\
& -\left(\frac{1}{N_{8}}\right) \int_{0}^{\infty} \sqrt{\xi F_{n n}\left[\xi\left(\frac{L_{R}}{L_{a}}\right)\right]}\left(-C_{1} F_{a}(\xi)+C_{2} \xi F_{a}^{\prime}(\xi)\right) d \xi \\
& -\left(\frac{1}{N_{8}}\right)_{0}^{\infty}\left[\sqrt{\xi F_{n n}\left[\xi\left(\frac{L_{R}}{L_{a}}\right)\right]}\left(-C_{1} F_{a}(\xi)+C_{2} \xi F_{a}^{\prime}(\xi)\right)\right] d \xi \\
& S_{0}\left(y, y^{\prime}\right)=\int_{0}^{\infty} F_{a}(\xi) S_{1}\left(y, y^{\prime}, \xi\right) d \xi \\
& S\left(y, y^{\prime}\right)=\left(\frac{L_{a}\left(y^{\prime}\right)}{N_{1}}\right) \int_{0}^{\infty}\left(\frac{1}{\xi}\right) F_{a}(\xi) S_{1}\left(y, y^{\prime}, \xi\right) d \xi-L_{R}(y) S_{0}\left(y, y^{\prime}\right) \\
& S_{1}\left(y, y^{\prime}, \xi\right)=\frac{\exp \left[-2 \xi\left|\frac{y^{\prime}-y}{L_{a}\left(y^{\prime}\right)}\right|\right]}{\int_{-\infty}^{+\infty} \exp \left[-2 \xi\left|\frac{y^{\prime \prime}-y}{L_{a}\left(y^{\prime}\right)}\right|\right] d y^{\prime \prime}}
\end{aligned}
$$

and

$$
\varepsilon=\frac{\mathrm{K} \sqrt{\mathrm{K}}}{\mathrm{L}_{\mathrm{R}}} .
$$

Note that the decay term due to molecular diffusion in the $b(y, t)$ has been omitted since we have neglected this effect in this study. Note the persistence of the nonlocality in both the transport equation for $K(y, t)$ and its length scale $L_{R}(y, t)$. This is an enhancement to the current formulation of the single-point BHR model equations. Another term that enhances the BHR model, as derived by Steinkamp (1995), is the density-mass-flux gradient source term to $b(y, t)$. 


\section{ACKNOWLEDGMENTS}

We are indebted to Rick Rauenzahn, Didier Besnard, and Murray Rudman for their insightful conversations over the past few years that have served to nurture the formulation of this work. Contributions and continuing interactions with Roy Axford, Lance Collins, Robert Kraichnan, Rodman Linn, Mat Maltrud, and Donald Sandoval have also proved valuable to this work and are much appreciated. We are also very grateful to Margaret Findley of T-3 for designing and preparing this manuscript.

\section{REFERENCES}

Andronov, V. A., S. M. Bakhrakh, E. E. Meshkov, V. V. Nikiforov, A. V. Pevnitshii, and A. I. Tolshmyakov, "An Experimental Investigation and Numerical Modeling of Turbulent Mixing in One-Dimensional Flows," Sov. Phys. Dokl. 27(5), 393-396 (1982).

Batchelor, G. K., The Theory of Homogeneous Turbulence, Cambridge University Press in the Cambridge Science Classics Series 1982, p. 38 (1986).

Bertoglio, J. -P., "A Model of Three-Dimensional Transfer in Non-Isotropic Homogeneous Turbulence," in Turbulent Shear Flows 3, L. J. S. Bradbury, F. Durst, B. E. Launder, F. W. Schmidt, and J. H. Whitelaw, Eds., (Springer-Verlag, 1982), pp. 253-261.

Besnard, D. C., F. H. Harlow, and R. M. Rauenzahn, "Conservation and Transport Properties of Turbulence with Large Density Variations," Los Alamos National Laboratory report LA10911-MS (1987).

Besnard, D. C., F. H. Harlow, R. M. Rauenzahn, and C. Zemach, "Spectral Transport Model for Turbulence, Los Alamos National Laboratory report LA-11821-MS (1990).

Besnard, D. C., F. H. Harlow, R. M. Rauenzahn, and C. Zemach, "Turbulence Transport Equations for Variable Density Turbulence and Their Relationships to Two-Field Models," Los Alamos National Laboratory report LA-12303-MS (1992).

Cambon, C., "Modélisation Spectrale En Turbulence Homogène Anisotrope," Thèse De DocteurIngénieru, Université Claude Bernard, Lyon (1979).

Cambon, C., D. Jeandel, and J. Mathieu, "Spectral Modelling of Homogeneous Non-Isotropic Turbulence, J. Fluid Mech. 104, 247-264 (1981).

Chandrasekhar, S., Hydrodynamic And Hydromagnetic Stability (Oxford University Press, Oxford, 1961), pp. 428-514.

Clark, T. T., Los Alamos National Laboratory, personal notes, 1994.

Clark, T. T., "Spectral Self-Similarity of Homogeneous Anisotropic Turbulence," Los Alamos National Laboratory report LA-12284-T (1992). 
Clark, T. T. and P. B. Spitz, "Two-Point Correlation Equations for Variable Density Turbulence," Los Alamos National Laboratory report, LA-12671-MS (1995).

Cranfill, C. W., "A New Multifluid Turbulent-Mix Model," Los Alamos National Laboratory document LA-UR-92-2484 (1992).

Daly, B. J. and F. H. Harlow, "Transport Equations in Turbulence," Phys. Fluids 13(11), 2634-2649 (1970).

Demuren, A. O., S. K. Lele, and P. Durbin, "Role of Pressure Diffusion in Non-Homogeneous Shear Flows," Bulletin of The American Physical Society. Program for the 1994 Annual Meeting of the Division of Fluid Dynamics 38(12), 1954 (1994).

Godeferd, F. S. and C. Cambon, "Detailed Investigation of Energy Transfers in Homogeneous Stratified Turbulence," Phys. Fluids 6(6), 2084-2100 (1994).

Harlow, F. H. and A. A. Amsden, "Fluid Dynamics," Los Alamos National Laboratory report LA-4700 (1971).

Heisenberg, W., "On the Theory of Statistical and Isotropic Turbulence," Proc. Roy. Soc. London A 195, 402-406 (1948).

Herring, J. R., National Center for Atmospheric Research, personal communication, 1994.

Karman, T. and L. Howarth, "On the Statistical Theory of Isotropic Turbulence," Proc. Roy. Soc. A164, 192-215 (1938).

Kovasznay, L. S. G., "Spectrum of Locally Isotropic Turbulence," J. Aeronautical Sciences. 745-753 (1948).

Kucherenko, Yu. A., L. I. Shibarshov, V. I. Chitaikin, S. I. Balabin, and A. P. Pylaev, "Experimental Study of the Gravitational Turbulent Mixing Self-Similar Mode," 3rd International Workshop on the Physics of Compressible Turbulent Mixing (Abbey of Royaumont, France, 1991), pp. 427-454.

Leith, C. E., "Diffusion Approximation to Inertial Energy Transfer in Isotropic Turbulence," Phys. Fluids 11, 671-673 (1967).

Leith, C. E., "Atmospheric Predictability and Two-Dimensional Turbulence," J. Atmos. Science 28(2), 145-161 (1971).

Lesieur, M., "Turbulence in Fluids," Second Revised Edition (Kluwer Academic Publishers, Dordrecht, 1990), pp. 146-152.

Nikiforov, V. V., "Calculation of Gravitational Turbulent Mixing in Non-Automodel Flows," 3rd International Workshop on the Physics of Compressible Turbulent Mixing (Abbey of Royaumont, France, 1991), preprint.

Nikiforov, V. V., All Russian Research Institute of Experimental Physics, Arsamas-16, personal communications, 1994. 
Read, K. I., "Experimental Investigation of Turbulent Mixing by Rayleigh-Taylor Instability," Physica 12D, 45-58 (1984).

Remington, B. A. and G. Dimonte, Lawrence Livermore National Laboratory, personal communications, 1995.

Sandoval, D. L., "Dynamics of Variable Density Turbulence," doctoral dissertation, University of Washington (1995).

Smeeton, V. S. and D. L. Youngs, "Experimental Investigation of Turbulent Mixing by Rayleigh-Taylor Instability, Part 3," AWRE report O 35/87 (1987).

Steinkamp, M. J., "A Spectral Model for Variable Density Turbulent Mixing," doctoral dissertation, University of Illinois (1995).

Youngs, D. L., "Modelling Turbulent Mixing by Rayleigh-Taylor Instability," Physica D 37, 270-287 (1989).

Youngs, D. L., "A Two-Dimensional Turbulence Model Based on the Equations of Multiphase Flow," in "Proceedings of the Third Zababakhin Scientific Talks," Kyshtym, USSR (January 14-17, 1992).

Youngs, D. L., United Kingdom Atomic Weapons Research Establishment, personal communications, 1993. 